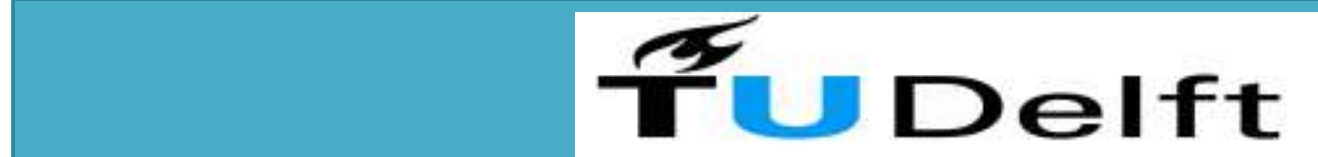

Delft university of Techmology

\title{
Inductive Power Transfer through Metal Object
}

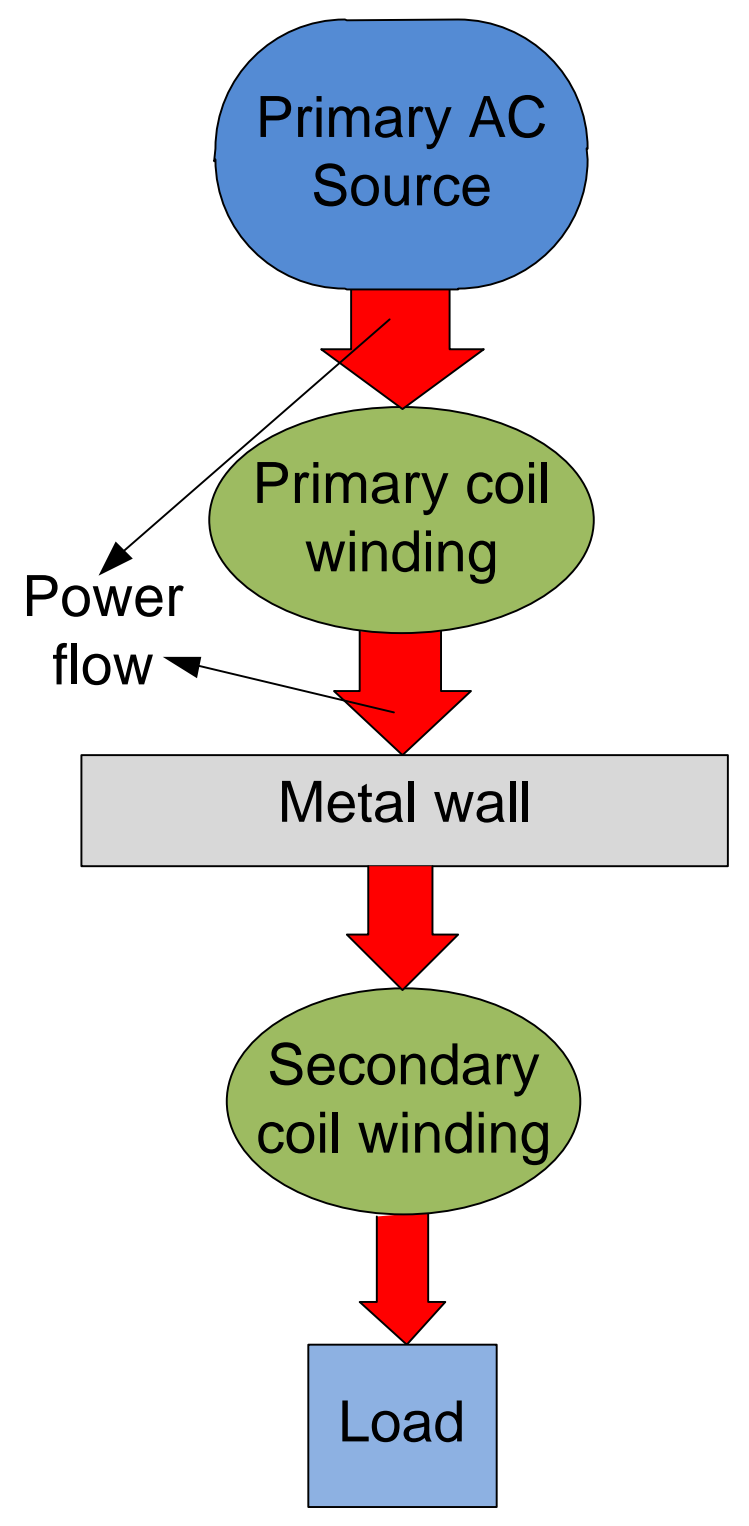

by

OdunAyo IMORU (1530267) 


\section{Inductive Power Transfer through Metal Object}

OdunAyo IMORU (1530267)

\section{for the degree of: \\ Master of Science in Electrical Engineering}

Date of submission: 20 July 2010

Date of defense: 3 August 2010

Committee:

Prof. dr. J. A. Ferreira

Dr.ir. H. Polinder

Dr. D.J.P. Lahaye

Ir. E. Nieuwkoop
Delft University of Technology (TU Delft)

Electrical Power Processing

Delft University of Technology (TU Delft)

Electrical Power Processing

Delft University of Technology (TU Delft)

Numerical Analysis

Netherlands Organization for Applied

Scientific Research (TNO Delft)

TNO Research

Electrical Engineering (Track-Electrical Power Engineering)

Faculty of Electrical Engineering, Mathematics and Computer Science

Delft University of technology, Delft 


\begin{abstract}
There are various situations where contactless/wiresless power transfer is desired. This could be due to safety, space, or other constraints. One such application is to supply power through a metallic pipe which could be part of a processing line. Such type of power transfer is called Inductive Power Transfer (IPT). The principle of IPT is very old but it is rarely used for transferring power from source to load via a conductive medium. This is because normally the medium restricts the power transfer due to losses or the shielding effects. However, for low energy applications, an acceptable amount of power could be transferred from the source to the load. This thesis aims at finding the amount of power transferred, the losses and efficiency for a given configuration. First, an analytical model is constructed. This analytical model involves equations governing the mechanism of IPT. It acts as a quick way to show what actually happens through the medium (metallic wall) placed between a primary coil (source) and secondary coil (load). This analysis leads to first approximation of the power transfer, losses in the medium and efficiency. Second, the thesis deals with experimental validation of the derived analytical model. A set up was made in the laboratory and certain experiments were performed for the comparison with analytical model. Experiments performed at different frequencies validated the analytical model but with some discrepancies, which are due to the assumptions made in the analytical model.
\end{abstract}




\section{Declaration}

I, OdunAyo Imoru do hereby solemnly declare that this thesis work "Inductive Power Transfer through metal object" presented for the award of Master of Science (MSc.) degree in Electrical Engineering is as a result of my personal effort. It has never been presented elsewhere either wholly or partially for either a degree or diploma. All the information used from published or unpublished works have been duly acknowledged. 


\section{Certification}

This is to certify that this thesis "Inductive Power Transfer through metal object" is the original work of OdunAyo Imoru carried out under the supervisions of Dr.ir. H. Polinder (EPP-TU Delft), Dr. D.J.P. Lahaye(Numerical Analysis-TU Deflt) and Ir. E. Nieuwkoop (Researcher-TNO Delft) for the award of Master of Science (MSc.) degree in Electrical Engineering Department, Delft University of Technology, The Netherlands. 


\section{Acknowledgments}

I acknowledge and remember the wonderful works of Almighty God upon me for His protection and compassion over me before and during this graduation thesis work. To Him be the glory and honour in Jesus name (Amen).

My greatest appreciation however, goes to my beloved wife Mrs Ronke-Ayo Imoru and my parents, Mr and Mrs F. O. Imoru for standing by me over the thesis period, and for words of encouragement. Thanks go to my family for their love and guidance, for supporting me through my international University education, and for their unceasing prayers in the last 24 months.

Many individuals have contributed either directly, or indirectly, to this thesis, and to the education I have received over the last two years. First and foremost, I am grateful to my supervisors, Dr.ir. H. Polinder (EPP-TU Delft), Dr. D.J.P. Lahaye(Numerical Analysis-TU Delft) and Ir. E. Nieuwkoop (Researcher-TNO Delft) for overseeing the work, for reading through many progress reports, for helpful guidance in preparation of the project reports, and for reviewing and editing the dissertation itself. I am also indebted to ir. Anoop K. Jassal, he has shown more concern to make this thesis a success despite his busy PhD research work. The time, words of encouragement and advices are greatly appreciated.

Special thanks also go to Pastor and Mrs Hyacinth Nwosu, Deacon and Mrs Alex Dawotola, Innocent Agbo, Avwaghwaruvwe Eguono, Sam Ani and TU Delft house fellowship members and MIZP RCCG members who helped me when I needed the spiritual assistance. In addition, I would like to thank my departmental friends for some discussions we had together helped me in my academic performance.

Finally, I would like to give my deep appreciation to The Netherlands government for granting me Netherlands Fellowship Programme (NFP) Scholarship award to study MSc Electrical Engineering, Delft University of Technology.

Ayo Imoru Delft University of Technology August, 2010 


\section{Dedication}

I dedicate this thesis to Almighty God and my beloved wife Mrs Ronke-Ayo Imoru 
COVER PAGE

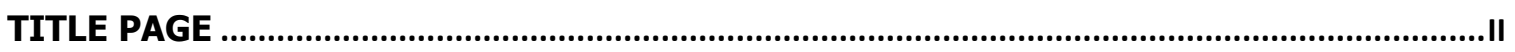

ABSTRACT

DECLARATION

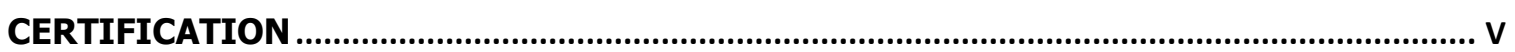

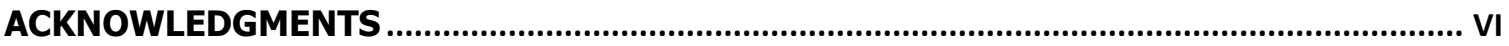

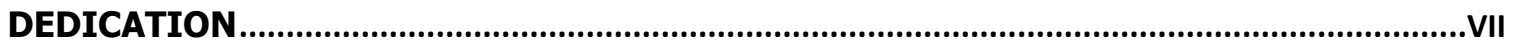

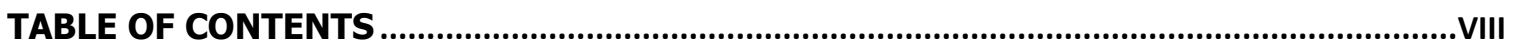

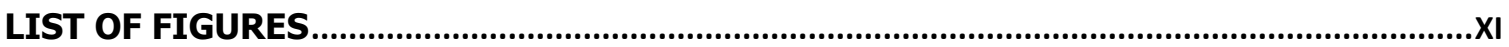

LIST OF TABLES …............................................................................................................................

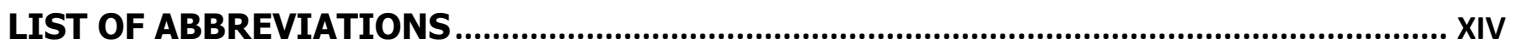

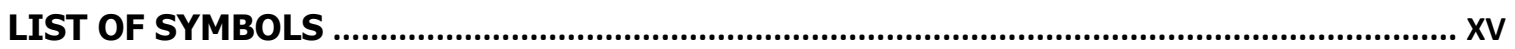

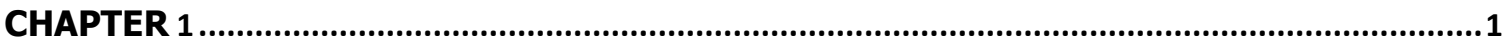

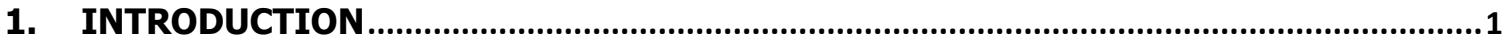

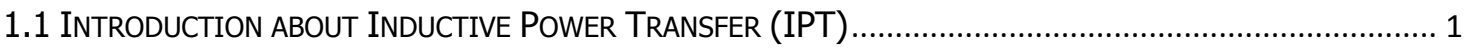

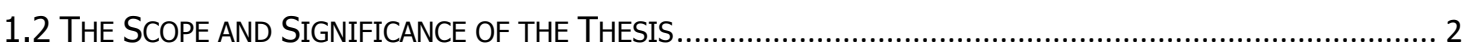

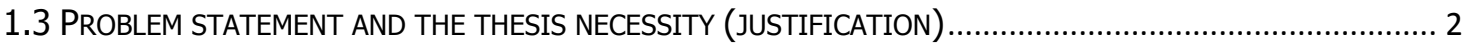

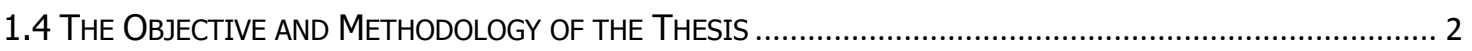

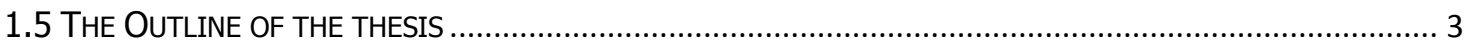

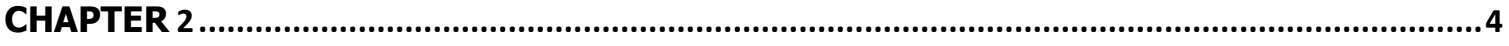

2. LITERATURE REVIEW ON INDUCTIVE POWER TRANSFER (IPT) ............................. 4

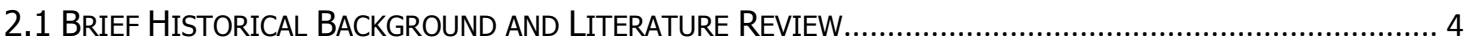

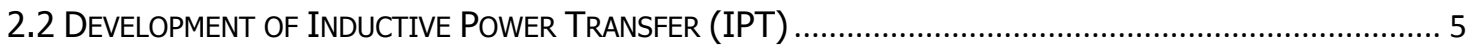

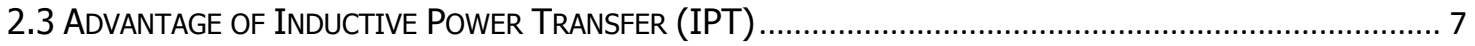

2.4 DiSAdVANTAGES OF INDUCTIVE POWER TRANSFER (IPT) …........................................................... 7

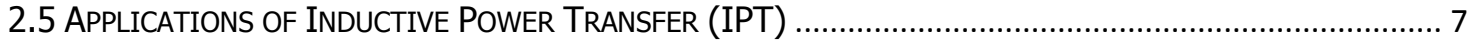

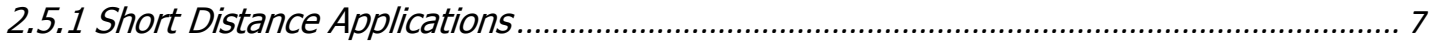

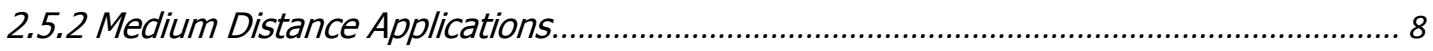

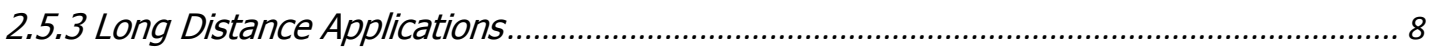

2.5.4 SmartStuds Applications (very long distance)................................................................ 8

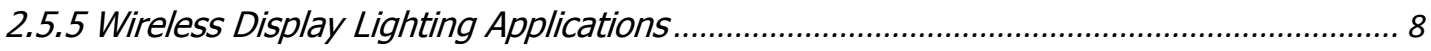

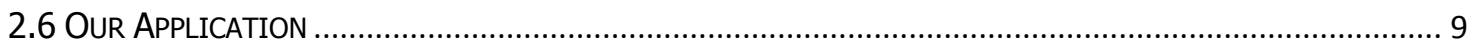

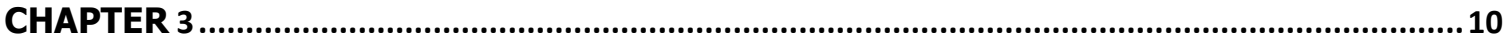

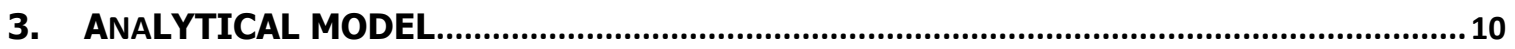




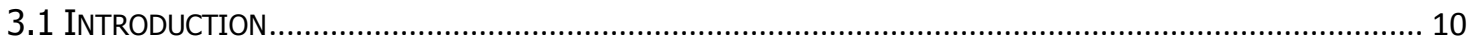

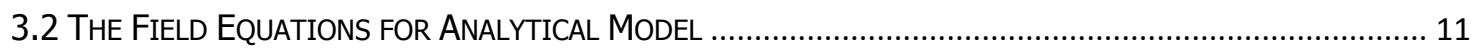

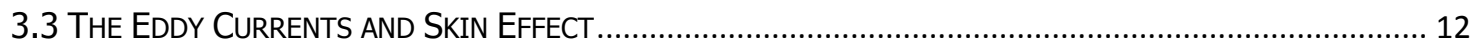

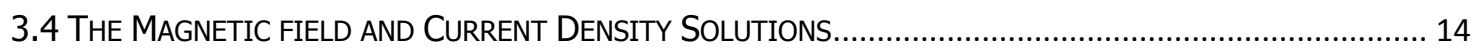

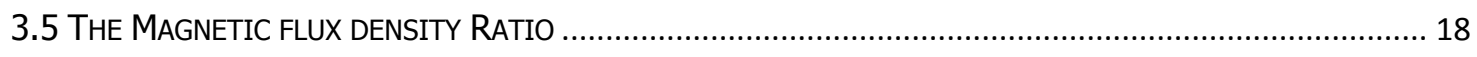

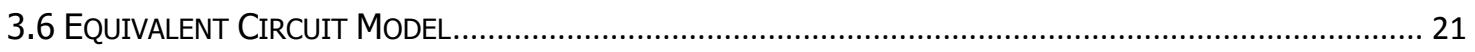

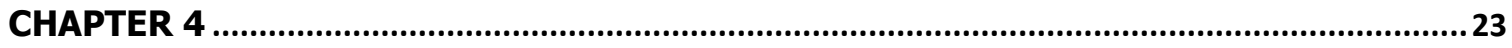

4. EXPERIMENTAL ANALYSIS AND DISCUSSION OF RESULTS ......................................23

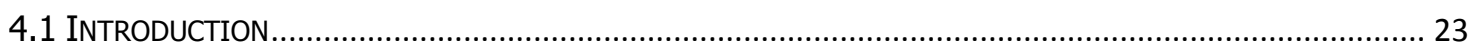

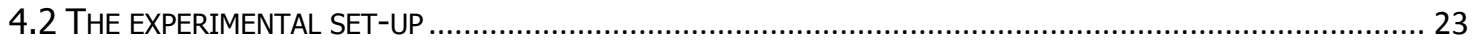

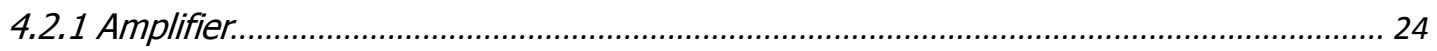

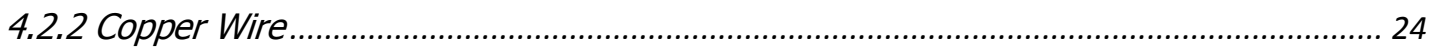

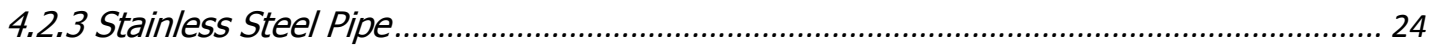

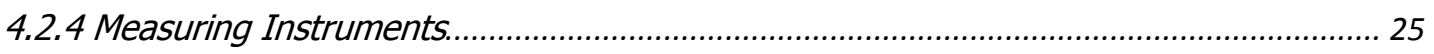

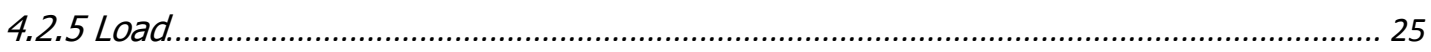

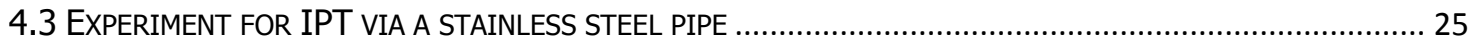

4.3.1 Number of turns required and Inductance Calculations................................................ 26

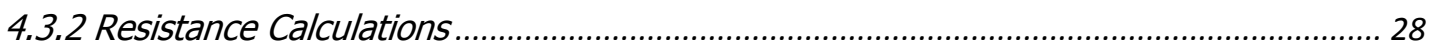

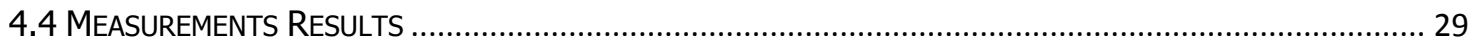

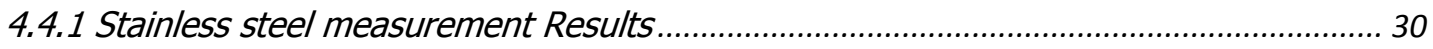

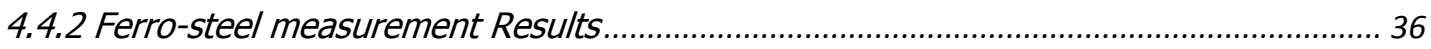

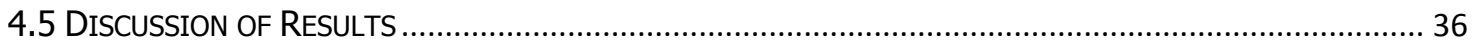

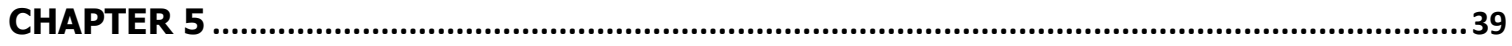

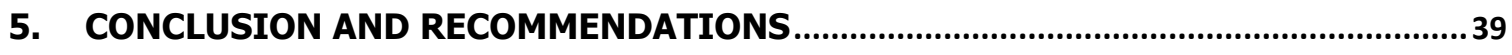

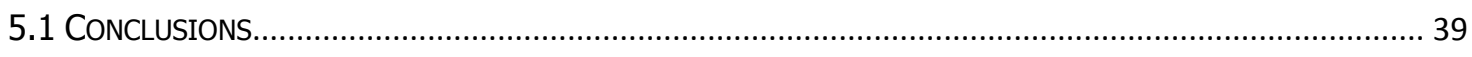

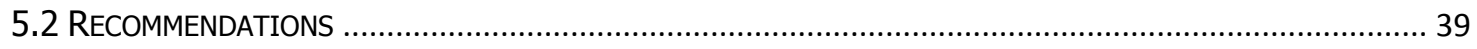

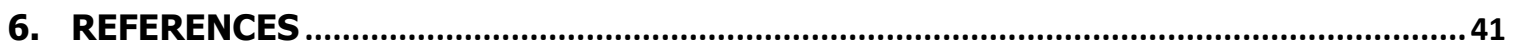

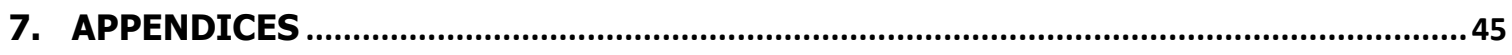

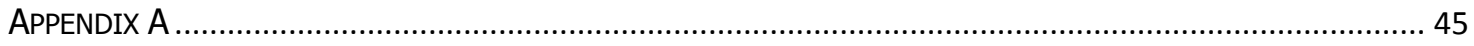

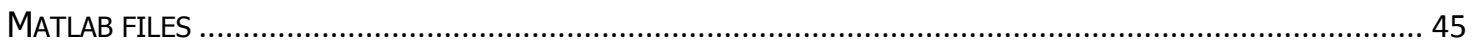

A1 Magnetic flux density and Current density as a function of thickness to skin depth ratio 45

A2: No-load (Induced and Magnetic flux) as a function of frequency..................................... 46

A3: Eddy current Losses at 40Hz when varying Load resistance............................................. 47

A4: Eddy current Losses at 50Hz when varying Load resistance.............................................. 49

A5: Eddy current Losses at 70Hz when varying Load resistance............................................ 51

A6: Eddy current Losses at $100 \mathrm{~Hz}$ when varying Load resistance............................................ 53

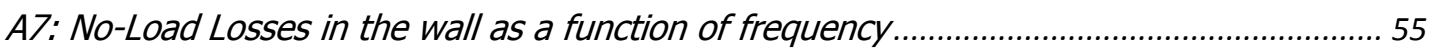

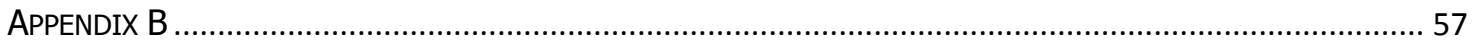

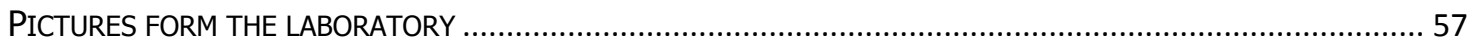

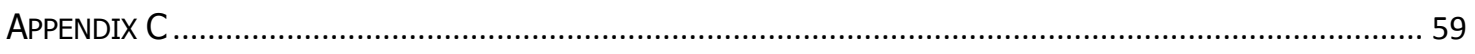

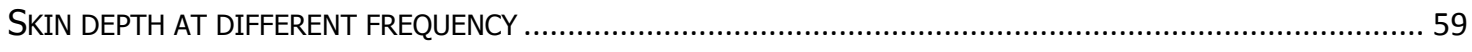




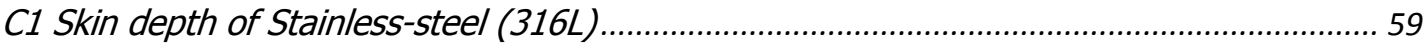

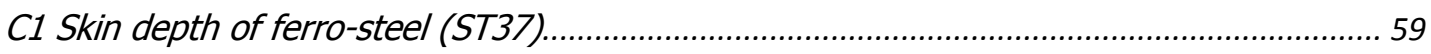




\section{List of figures}

Figure 1.1: Block diagram showing Components of IPT system via a metallic wall ................... 1

Figure 2.1: 1887 experimental set-up of Hertz's apparatus [3] ........................................ 4

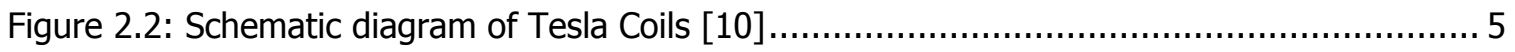

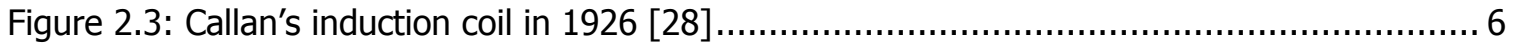

Figure 2.4: Primary and secondary coils are aligned with B-field perpendicular to the wall ..........9

Figure 3.1: Cylindrical compartment (oil-pipe) with primary and secondary coils aligned and

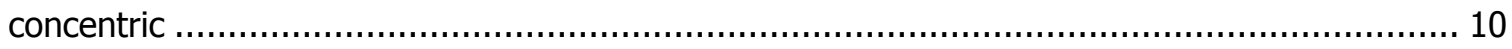

Figure 3.2: The penetration of $\mathrm{H}$, having the same tangential component just outside and inside

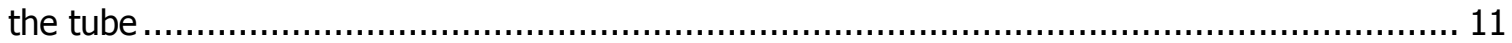

Figure 3.3: Magnetic flux density variations magnitude with skin depth ............................. 15

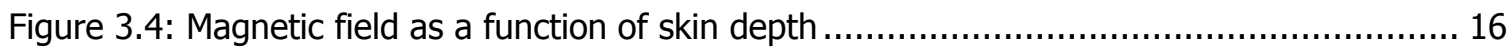

Figure 3.5: Current density as a function of skin depth ................................................... 17

Figure 3.6: Metal pipe with primary coil aligned and concentric ........................................ 18

Figure 3.7: Metal pipe wall with primary coil connected to power source and Secondary to coil

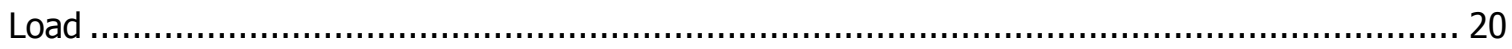

Figure3.8: Equivalent model of figure 3.7 without load ................................................. 21

Figure 4.1: Preliminary experiment set up showing the coils (Primary and Secondary) on the pipe

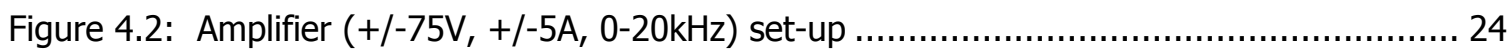

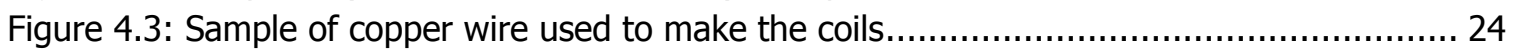

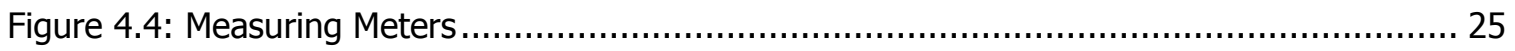

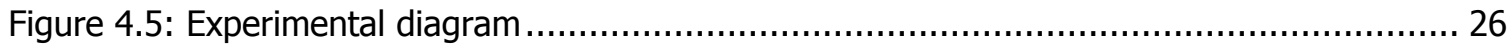

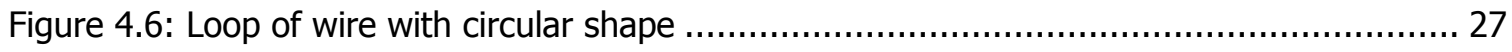

Figure 4.7: Tube section covered by the coils ........................................................... 29

Figure 4.8: (a) Plot of Input Power, output Power and efficiency against Load at 40Hz.,(b) Plot of

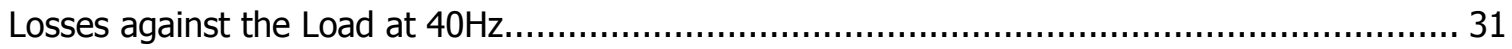

Figure 4.9: (a) Plot of Input Power, output Power and efficiency against Load at $50 \mathrm{~Hz}$.,(b) Plot of

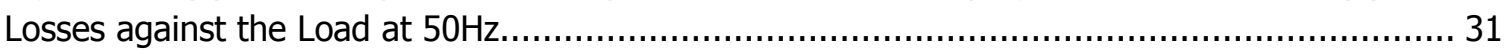

Figure 4.10: (a) Plot of Input Power, output Power and efficiency against Load at 70Hz.,(b) Plot

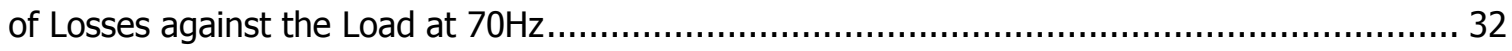

Figure 4.11: (a) Plot of Input Power, output Power and efficiency against Load at $100 \mathrm{~Hz}$. ,(b) Plot

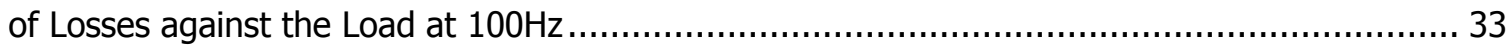

Figure 4.12: (a) Plot of Input Power, output Power and efficiency against frequency at $5.55 \Omega$ (b)

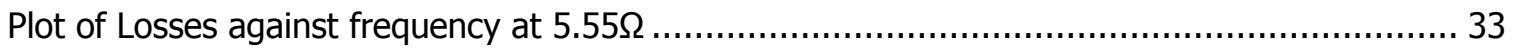

Figure 4.13: Plot Magnetic flux density against frequency at no-load ............................. 35

Figure 4.14: comparison of experimental and analytical plots of magnetic flux density against

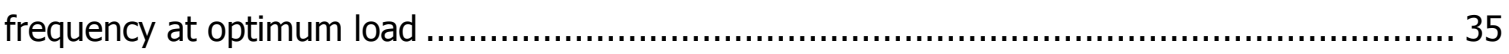

Figure 4.14: comparison of experimental and analytical plots of losses against frequency......... 37

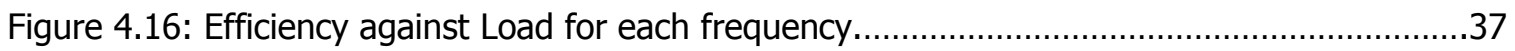

Figure A.1: Analytical value of Magnetic flux as a function of frequency at no-load................. 47

Figure A.2: Analytical value of Induced voltage as a function of frequency at No-Load ............ 47

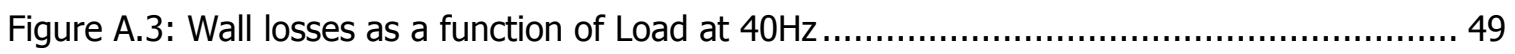

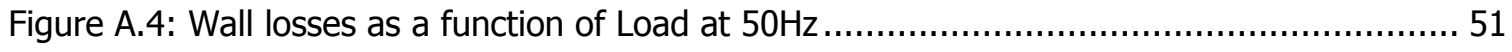

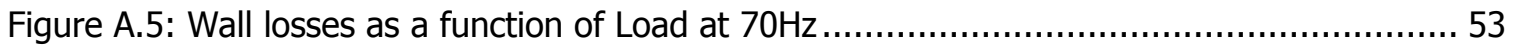

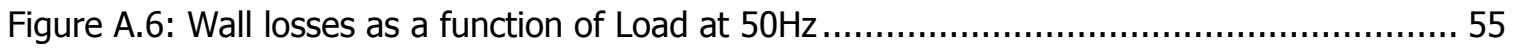




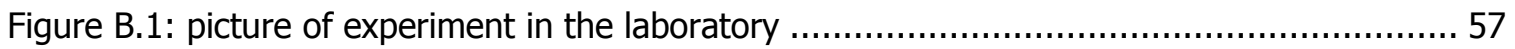

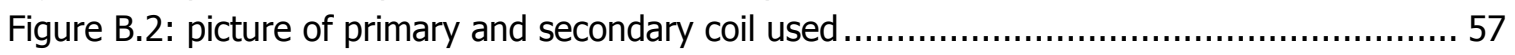

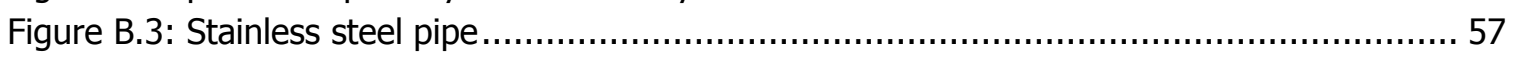

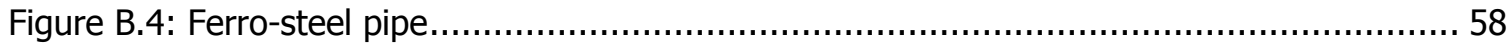




\section{List of tables}

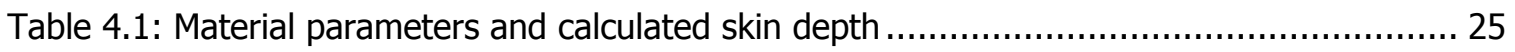

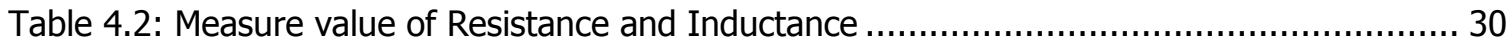

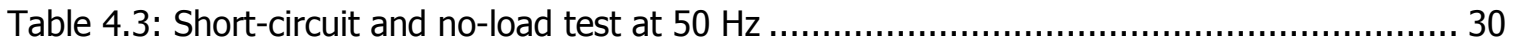

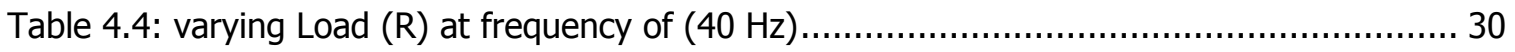

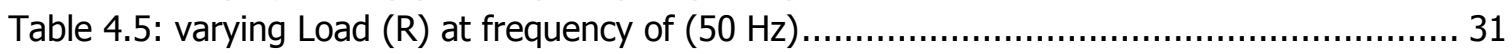

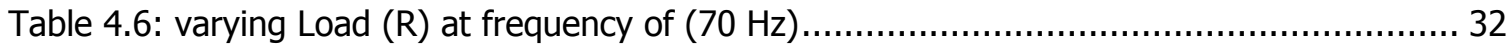

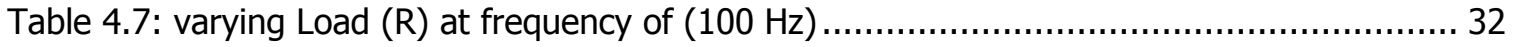

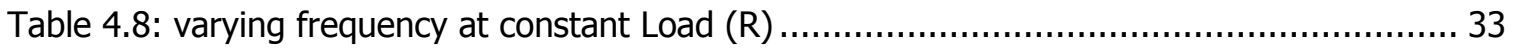

Table 4.9: No-load measurements at different frequency .............................................. 34

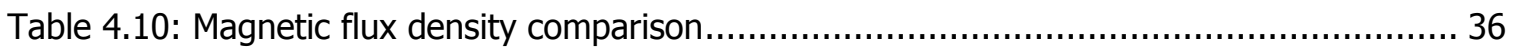

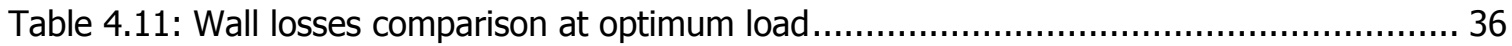

Table C.1: skin depth of Stainless steel at frequency considered ....................................... 59

Table C.1: skin depth of Ferro-steel at frequency considered ........................................... 60 


\section{List of abbreviations}

IPT-Inductive Power Transfer

AC-Alternating Current

DC-Direct Current

LEDs-Light Emitting Diodes

FEM- Finite Element Methods 


\section{List of symbols}

\section{Symbol}

$\vec{B}$

$B_{x}$

$B_{s}$

$\vec{J}$

$J_{s}$

$\mu$

$\mu_{r}$

$\mu_{0}$

$\vec{E}$

$\vec{H}$

$\vec{i}$

$r$

$\sigma$

$t$

$\delta$

$N$

I

$h$
Quantity

Magnetic flux density

x component of $\vec{B}$

Magnetic flux density at the surface

Current density

Current density at the surface

Magnetic permeability

Relative magnetic permeability

Magnetic permeability in vacuum

Electric field strength

Magnetic field strength

Unit vector

Relative (in the magnetic permeability $\mu_{r}$ )

Conductivity

Time

Skin depth

Number of turns of coil

Current

Thickness of the pipe

\section{Unit}

$\mathrm{T}$

$\mathrm{T}$

$\mathrm{T}$

$\mathrm{A} / \mathrm{m}^{2}$

$\mathrm{A} / \mathrm{m}^{2}$

$4 \pi \times 10^{-7} \mathrm{H} / \mathrm{m}$

$\mathrm{V} / \mathrm{m}$

$\mathrm{A} / \mathrm{m}$

$\mathrm{S} / \mathrm{m}$

S

m

A

m 
$R$

$\mathrm{R}$

$\lambda$

$L$

M

$\omega$

$f$
Resistance

Radius

Flux linkage

Inductance

mutual inductance

Angular frequency

Frequency
$\Omega$

m

Wb

$\mathrm{H}$

$\mathrm{H}$

$\mathrm{rad} / \mathrm{s}$

$\mathrm{Hz}$ 


\section{Chapter 1}

\section{Introduction}

This chapter presents the brief introduction about the inductive power transfer, the scope and significance of the thesis (inductive power transfer via a metal). The problem statements and the necessity (justification) are also discussed. The aim and methodology of the thesis are briefly elucidated before the general outlines of the thesis are presented in the last part.

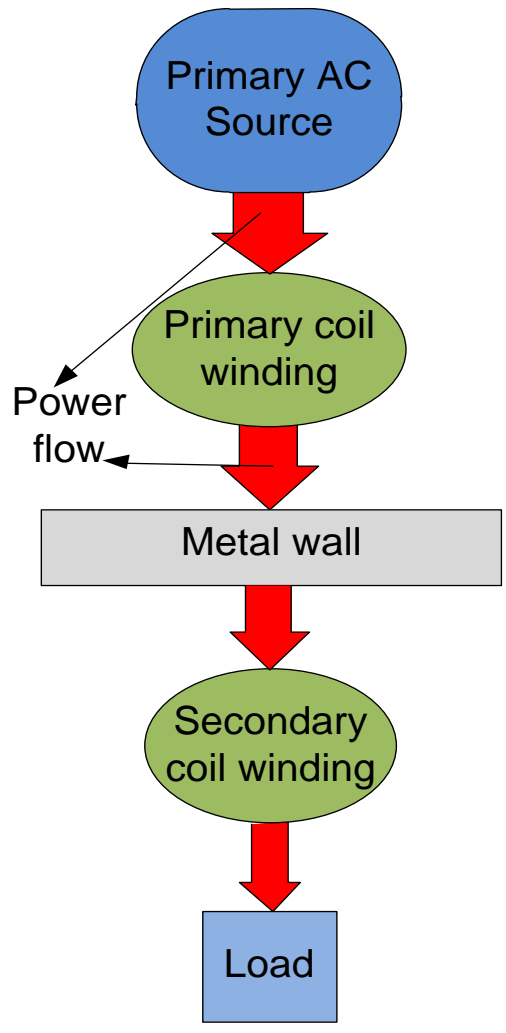

Figure 1.1: Block diagram showing Components of IPT system via a metallic wall

\subsection{Introduction about Inductive Power Transfer (IPT)}

There are various situations in which electrical energy is desired but cannot be conveniently supplied. In [1], Vandevoorde celebrated an inductive link that transferred $20 \mathrm{~W}$ of power over a distance of 1 centimetre $(\mathrm{cm})$ with an overall efficiency of $80 \%$. It is justified from [2] that the technology of the Inductive Power or Energy Transfer has also changed dynamically after the increase in the use of (wireless) sensors and actuators. In a number of cases, controlling units and power supplies are separated from the sensors and actuators by metal object or metal compartment (for example, oil pipe). Drilling holes through the metal object for power and signal feed through it is never always an acceptable solution due to potential leakage risk or weakening 
of the metal object as in case of high pressure. However, with inductive power or signal transfer through a metal object, these problems could be circumvented.

The structure of an inductive power transfer system via a metal compartment (wall) is very simple (see the block diagram in Figure 1.1). The basic structure consists of a primary AC source, a primary winding, a secondary winding, a metal wall and the load output. The power flow is also included in the figure (1.1) which means Inductive power is transferred from the primary to the secondary winding via a thin metallic wall to the load.

\subsection{The Scope and Significance of the Thesis}

Inductive power transfer allows the transfer of electrical power without physical contact i.e. without interconnecting wires. With no-contact means contactless and this is synonymous to wireless. This Wireless energy transfer is different from wireless transmission of information, such as radio, where the signal-to-noise ratio or the percentage of power received becomes critical only if it is too low to recover the signal successfully. With wireless energy transfer, efficiency is more important parameter. Therefore the direct linkage of magnetic field between primary and secondary coils is required. In the present-day technologies, wireless power transfer is carried out using induction, but some other wireless power transfer methods are highlighted in Chapter two.

\subsection{Problem statement and the thesis necessity (justification)}

In figure 1.1, effective power transfer via the metal pipe (wall) material given is very important because the wall thickness and type of material determine the amount of power transferred to the secondary coil. One of the application fields of these devices is inside steel (process) pipes. In that case the power supply is of special concern. This is because in a number of cases where batteries are used, the energy content is too low and batteries have a limited lifetime. Since the energy yield will be too low for various applications even if energy scavenging techniques exits, Inductive Power Transfer (IPT) could be used to solve these problems.

\subsection{The Objective and Methodology of the Thesis}

The objective of this thesis is to explore the possibilities of transferring power inductively from an energised primary coil winding via a metallic cylindrical pipe (wall) to a secondary coil winding. Besides that, we will also look into how wall (pipe) thickness and materials influence the inductive energy transfer via any medium (pipe) and to determine the optimum load for maximum power transfer. This is achieved by considering analytical model that involves equations governing the mechanism of inductive power transfer. The analytical method is quick way only to validate what actually happens through the wall, however we will confirm it with experimental model to see if it is reliable. 


\subsection{The Outline of the thesis}

The contents of the thesis are arranged as follows:

In Chapter 1, the introduction, we talk about the scope and the importance of the thesis including the problem statements and the necessity (justification). Also, the aim and methodology of the thesis are briefly discussed

Chapter 2 tells us about the historical background and literature review of inductive power transfer(s). It also reveals the development of IPT, advantages, applications and the principle behind the thesis design methodological concept.

In Chapter 3, we look into the analytical model of the design adopted. It was validated that contactless (inductive) power could be transferred from a primary coil outside a cylindrical metal wall to secondary coil inside the cylinder.

Chapter 4 discusses the experimental set-up, results obtained and comparisons of these results with the analytical.

In Chapter5, the conclusions from results and applications of the thesis work are discussed with recommendations for future research. 


\section{Chapter 2}

\section{Literature Review on Inductive Power Transfer (IPT)}

This chapter describes some of the historical background and literature review of inductive power transfer(s). It also reveals the development of IPT, advantages, applications and the principle behind the thesis design methodological concept

\subsection{Brief Historical Background and Literature Review}

On early history of contactless (wireless) power transfer, two pioneers Heinrich Rudolf Hertz (February 22, 1857 - January 1, 1894) and Nikola Tesla (10 July 1856 - 7 January 1943) came out with substantial results after wireless power transfer has been attempted persistently all through the last two centuries[3],[4].

This idea started with Heinrich Hertz when he confirmed the existence of electromagnetic radiation in his experiment in 1888. In [5] Cichon discussed how he (Hertz) proved that electricity can be transmitted in the form of electromagnetic waves. Figure 2.1 shows this experiment as he made observations of the photoelectric effect and of the production and reception of electromagnetic (EM) waves. His receiver consisted of a coil with a spark gap, where upon a spark would be seen upon detection of EM waves. Brown in [6] described how Hertz also created an experiment to generate high frequency power and detected it at the receiving end in [2.4].

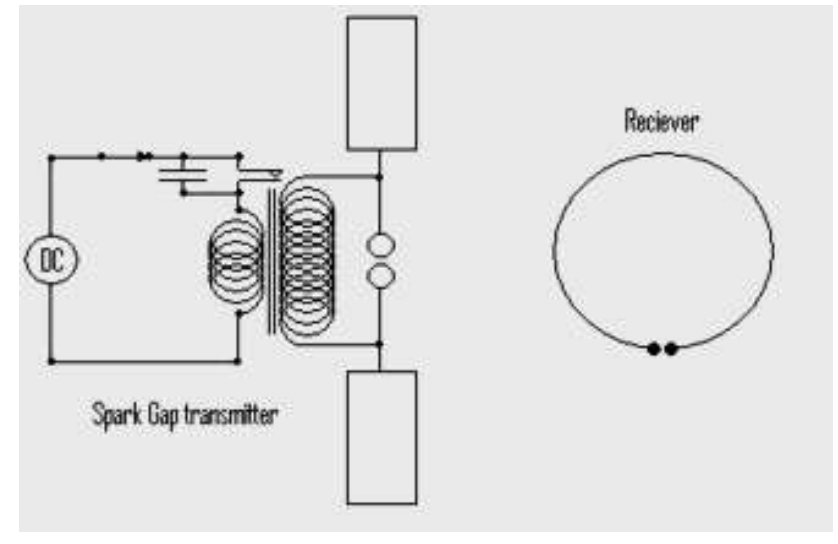

Figure 2.1: 1887 experimental set-up of Hertz's apparatus [3]

Nikola Tesla was another pioneer who had an exceptional talent of being able to imagine things so that they looked original. This allowed him to build mental rather than physical prototypes that led to successful finished designs. He had more foresight beyond his time in his Experiments. The limitation was that Tesla took very poor notes and only wrote down those things that he considered extremely necessary. It was not until 1970 that Robert Golkabe [7] became the first to replicate the Tesla coil. Other experiments of Tesla are yet to be reproduced 
[8]. A typical example of a simplified schematic diagram of Tesla coil is shown on figure 2.2. We can read more about Maxwell's contribution in [9] where Simpson wrote an article on Maxwell and the direct experimental test on his electromagnetic theory

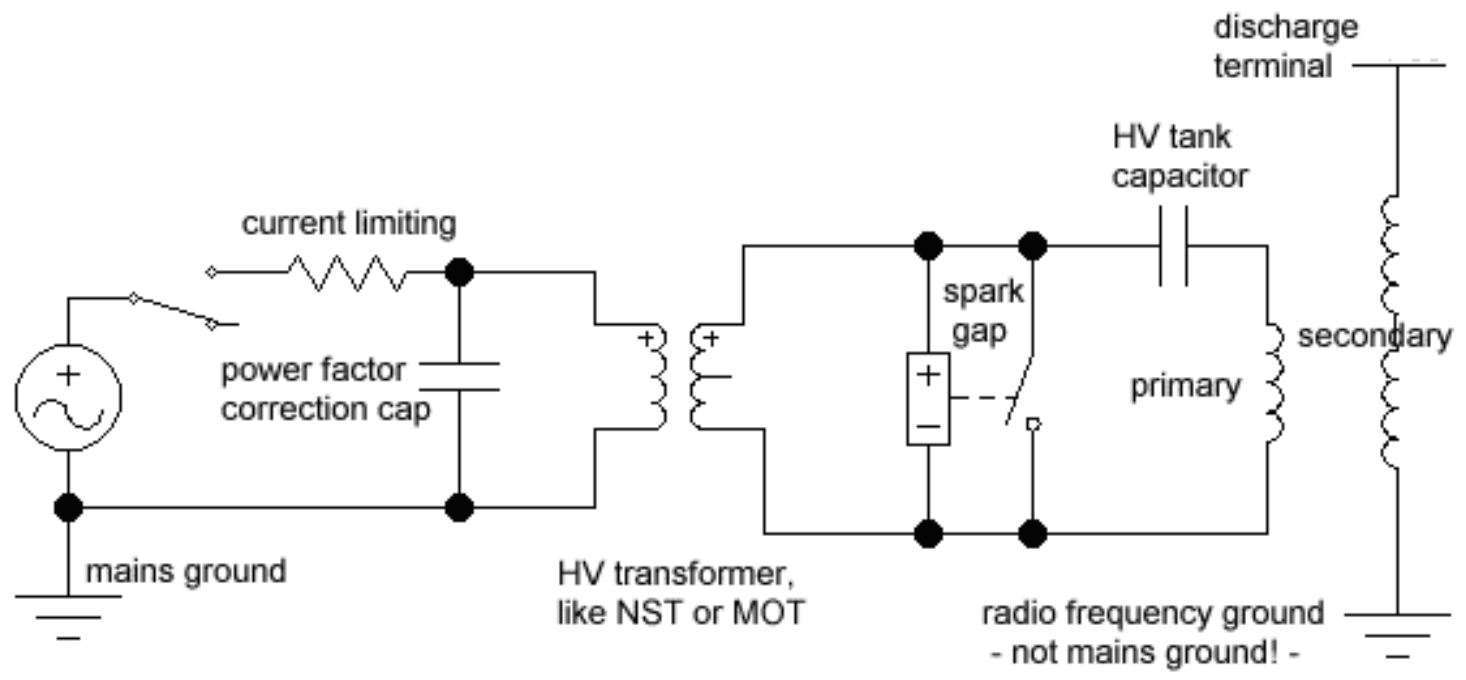

Figure 2.2: Schematic diagram of Tesla Coils [10]

Tesla was confident in his hypothesis to transfer power and it has been confirmed according to [9], but power transferred was too low. These days, wireless power transfer is largely exhibited through induction.

An inductive (contactless) power transfer system operates much like an ordinary transformer, but with only a medium between the coils. The medium could be magnetic or non-magnetic. There has been several publications like $[1],[2],[11],[12],[13],[14]$ and [15] on power transfer via non-magnetic medium between two coils, whereas power transfer via a ferromagnetic medium is a very new idea and this needs thorough investigations for breakthrough. Examples of contactless power transfers with breakthroughs include contactless energy transfer in hazardous or wet surroundings[11], contactless electric vehicle battery charger [15-17], roadway power electric vehicles [18-20], robotic applications[21,22],sliding transformers[23] and underwater power transfer[24]. Although functional, wireless power transfer through induction is constrained to small distances; the transfer efficiencies get increasingly worse as the distance between transmitter and receiver increases. We shall also look into the development of inductive power transfer, the benefits, and applications in the next section.

\subsection{Development of Inductive Power Transfer (IPT)}

Electromagnetic induction is an old phenomenon, so inductive power technology resulted from combining these two inventions and discoveries. 
i. Electromagnet - in 1825 by William Sturgeon invented the electromagnet a conducting wire wrapped around an iron core [25]

ii. Induction - a changing magnetic field can induce an electrical current in an adjacent wire was discovered by Michael Faraday in 1831 [26]

By combining these two discoveries, Nicholas Joseph Callan (1799-1864) first demonstrated the transmission and reception of electrical energy without wires in 1836 [27]. Callan's induction coil (figure 2.3) consisted of two insulated coils (primary and secondary windings) both placed around a common iron core. A battery intermittently connected to the primary would 'induce' a voltage in the longer secondary causing a spark to jump across its free terminals.

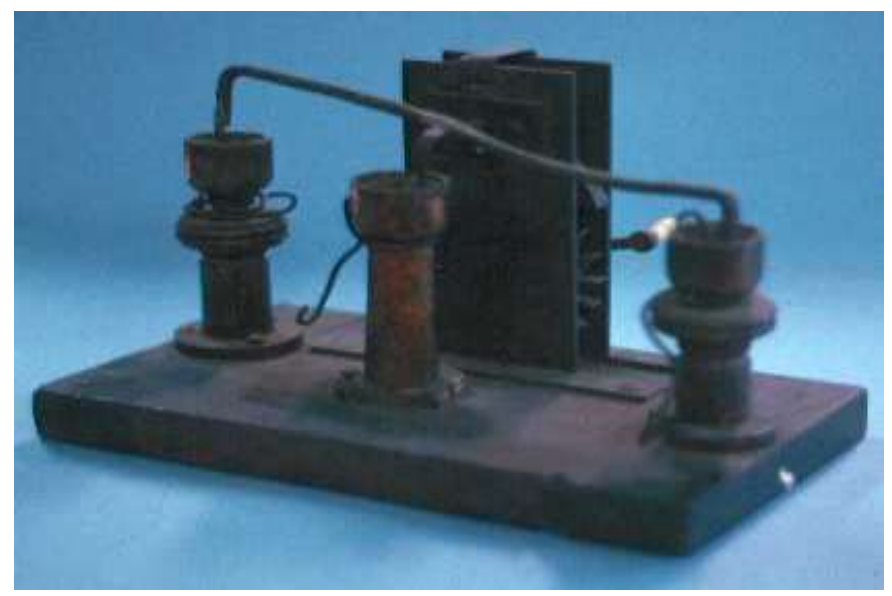

Figure 2.3: Callan's induction coil in 1926 [28]

In an induction coil or electrical transformer, which can have either an iron core or an air core, the transmission of energy takes place by simple electromagnetic coupling through a process known as mutual induction. Using this method it is possible to transmit and receive considerable energy, but two inductors must be placed fairly close together to draw significant power by induction.

As wireless technologies were further developed during the early $1900 \mathrm{~s}$, different wireless transmission methods were further investigated with the goal to generate an effect locally and detect it at a distance. Efforts were also made to power more significant loads than the highresistance sensitive devices that were being used to simply detect the received energy

Therefore, there is a potential interest to know that the principles and developments need further research that can be linked to this thesis. Inductive power transfer has some advantages over other means of power transfer, so we shall look into the advantages as a reason to investigate further. 


\subsection{Advantage of Inductive Power Transfer (IPT)}

The benefits of IPT include:

- Long life - with virtually no components prone to wear and tear, the service life of the system is greatly increased. There is no friction in the system, hence no limit to the acceleration possible. With no galvanic contact, there is no corrosion.

- Safety - unlike wire power-sources that have the risk of sparks from static electricity or friction, IPT provides power to environments previously considered too difficult or previously accepted as a hazard.

- Low Maintenance - there are no batteries to be replaced.

- No wear and Tear

- No pollution

\subsection{Disadvantages of Inductive Power Transfer (IPT)}

The limitations of IPT include:

- Medium of transfer can get heated up at high frequency

- Conductive medium with high magnetic permeability will limit the power transfer

\subsection{Applications of Inductive Power Transfer (IPT)}

Besides the specific application examples mentioned in section 2.1, there are a number of current applications for IPT, categorized by the distance of the power induction. Therefore, applications of short distance power transfer which is basically IPT and other applications of contactless power transfer like Medium distance, Long distance , SmartStuds e.t.c which are wireless will be discussed

\subsubsection{Short Distance Applications}

The following are short distance applications of Inductive power transfer..

- The electric toothbrush battery charger [12]

- The induction cooker stovetop [29]

- Surgical Implants - Transcutaneous energy transfer (TET) systems in artificial hearts like AbioCor and other surgically implanted devices.[30]

- Consumer Electronics - devices using induction to charge portable consumer electronics such as cell phones.[31]

- Recharging electric cars/buses

- Sealless Valve- inductive(wireless) energy transfer via a pipe wall (this thesis) 


\subsubsection{Medium Distance Applications}

The medium distance applications of wireless power transfer include:

- a wireless power transfer using Radio Frequency (RF) energy. This was introduced by a new company called Powercast Corporation [32] at the 2007 Consumer Electronics Show, winning best Emerging Technology. The Powercast system is applicable for a number of devices with low power requirements. This could include (i) LEDs, (ii) computer peripherals, (iii) wireless sensors, and (iv) medical implants. Currently, it achieves a maximum output of 6 volts for a little over one meter

- A different low-power wireless power transmission using Laser was proposed by Landis [33]. Readers [34, 35] can be refereed to for further study.

\subsubsection{Long Distance Applications}

Long distance applications of wireless power transfer incorporate:

- Power transmission via radio waves which can be made more directional by allowing longer distance power beaming with shorter wavelengths of electromagnetic radiation typically in the microwave range. A practical wireless energy transmission system using this principle may use a high-power ultraviolet beam to form a vertical ionized channel in the air directly above the transmitter-receiver stations. This concept is used in (i) virtual lightning rods, (ii) the electro-laser electroshock weapons and has been proposed for (iii) disabling vehicles. [36, 37]

\subsubsection{SmartStuds Applications (very long distance)}

This application can be seen in what a New Zealand company called Harding Traffic developed. This is a road lighting system, called Smartstuds [38, 39]. Lights are glued to the road above a cable placed just below the surface. The power is then transferred using induction between the lights and the cable. This installation configuration is cheaper and offers more flexibility than other power light systems. Programming configurations allow for custom display options such as stud colour and the ability to remotely make changes to signal lane changes or hazards. (For example, lights turn blue to show an ice hazard). Smartstuds are now rapidly being installed in Europe, Asia and the United States for tunnels, bridges, roads, walkways and pedestrian crossings.

\subsubsection{Wireless Display Lighting Applications}

Inductive Power Transfer technology [37] is also well suited for use in wireless display lighting and these include:

- Neon and Argon Signs: Neon and Argon signs require very high strike voltages of several $\mathrm{kV}$. These are easy to generate with IPT systems so the tubes are easy to light 
and will operate in wet and dirty environments without difficulty. They may be switched on/off at speed to give a controlled brightness using low cost transistor switches. This option is not possible at $50 / 60 \mathrm{~Hz}$.

- Very large displays: Very large displays for instant replays at sports functions and the like may be made completely wireless using IPT power supplies. The information to be displayed is coupled to the displays by radio signals. The power to drive display modules is coupled by IPT. The modules can be made completely waterproof with an IP65 rating they can be operated continuously in a tropical downpour. The whole display has no connectors, no sockets, and no exposed wires. Modules can also be made self-addressing so that they may be placed anywhere in the display and automatically display the appropriate part of the picture with colour and brightness matching across the whole display.

\subsection{Our Application}

The idea presented here is to transfer the required energy inductively through the wall in fig 2.4. The principle of inductive power transfer (IPT) is very old. The reason for this is not farfetched because it is hardly used for this type of application due to either a high magnetic permeability of the metal wall that forms a shield for the inductive field and high eddy currents inside the pipe's wall. In this thesis, a Stainless steel pipe material would be considered as wall between the two coils (primary and secondary) because it is very conductive with low permeability. With a decreasing power demand of modern sensors, even a low energy transfer rate could lead to an acceptable amount of power transfer to the opposite side of the wall, however it is expected that most of the energy will be dissipated in the wall due to hysteresis and eddy-current losses.

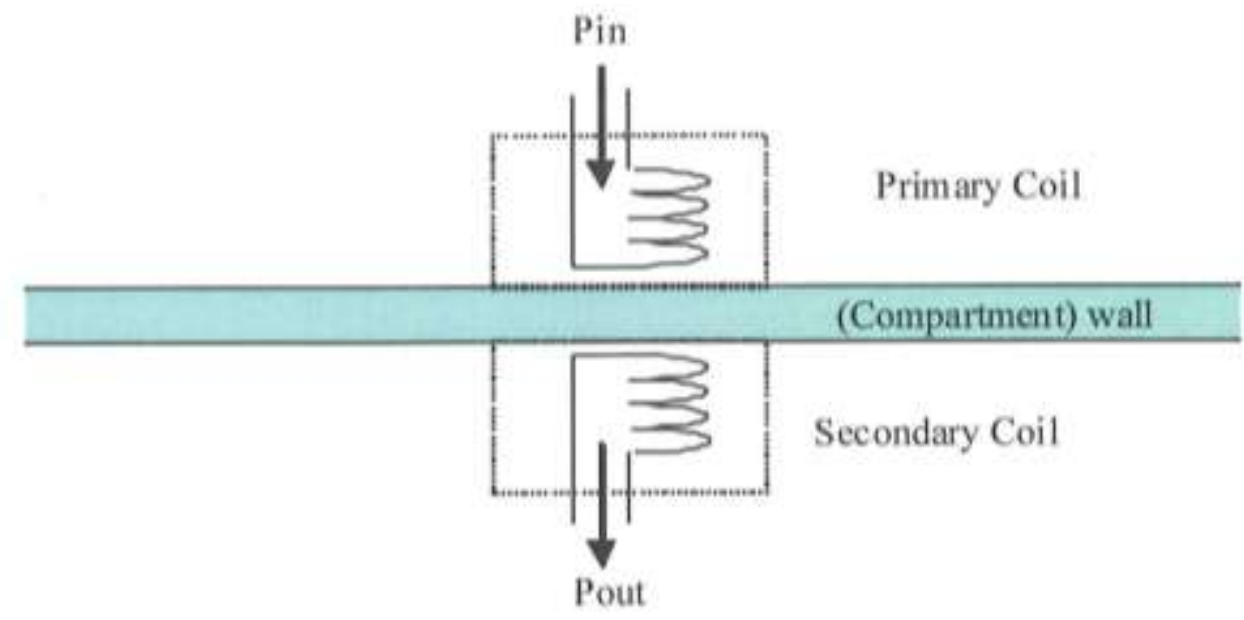

Figure 2.4: Primary and secondary coils are aligned with B-field perpendicular to the wall 


\section{Chapter 3}

\section{Analytical Model}

In this chapter, we look into the analytical model where we solve the magnetic field equations resulting in finding solutions to magnetic flux density and current density equations. We shall also discuss the ratio of magnetic field at the surface of the wall to the magnetic field inside the wall and lastly make a circuit model which can be used for further investigation.

\subsection{Introduction}

The objective of transferring power from primary coil to secondary coil via a metallic pipe wall needs some analytical investigation before we set up an experiment to validate it. We need to prove if it is really possible to transfer power via the metallic wall analytically. Analytical modelling is quick but it is too difficult to capture all the geometrical effects and magnetic non-linearity of the material. Therefore some assumptions are made to arrive at logical conclusion. It is shown analytically that contactless inductively power transfer from primary coil to secondary coil via a cylindrical metal (steel) tube (like an oil-pipe) as in fig 3.1 can be achieved. The primary and the secondary coils are separated by the (compartment) wall and the magnetic field in this case is parallel to the (enclosure) wall. A ferromagnetic material housing may also be included to the coils to improve the efficiency and to make the analysis much easier.

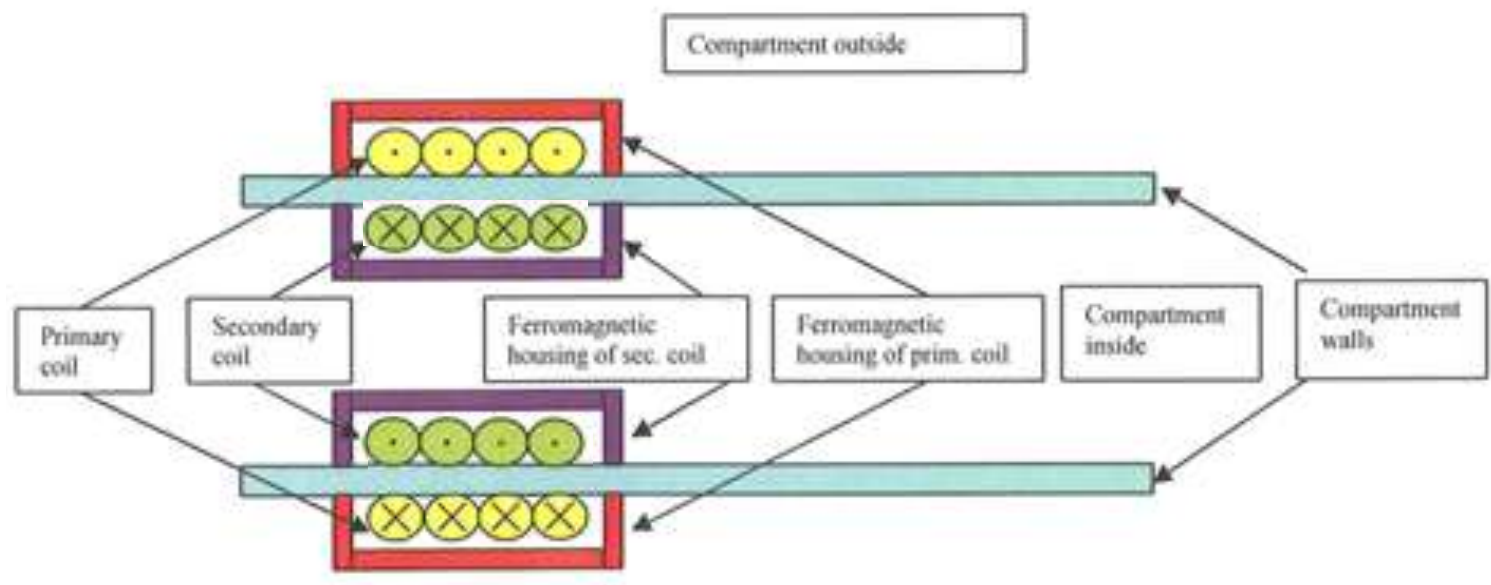

Cotiparment outule

Figure 3.1: Cylindrical compartment (oil-pipe) with primary and secondary coils aligned and concentric

In the first part of the analysis figure 3.2 which is based on the understanding of previous figure 3.1 is used. The figure contains a cylindrical tube (say stainless steel oil-pipe) with 
energized primary coils where currents going into the paper and cause an induced eddy current in the wall of the pipe and at the inside of the pipe we have the secondary coil.

\section{Assumptions}

The assumptions made in fig 3.2 are as follow:

- One-dimensional situation is considered and a rectangular coordinate system is used in this analysis. The magnetic field (B) is parallel to the wall in $x$-direction and the Electric (E) field is also parallel to the wall, but in z-direction (i.e at mutually perpendicular to $\mathrm{B}$-field in $\mathrm{x}$-direction). Both fields are function of $(y)$

- Nonlinear effects, such as hysteresis and saturation phenomena, are neglected.

- The tube is assumed to be infinitely long so that end effects are also neglected.

- An ideal iron (ferromagnetic) core forming housing for primary coil is included. The core is assumed to have an infinite permeability $\left(\mu_{r}=\infty\right)$

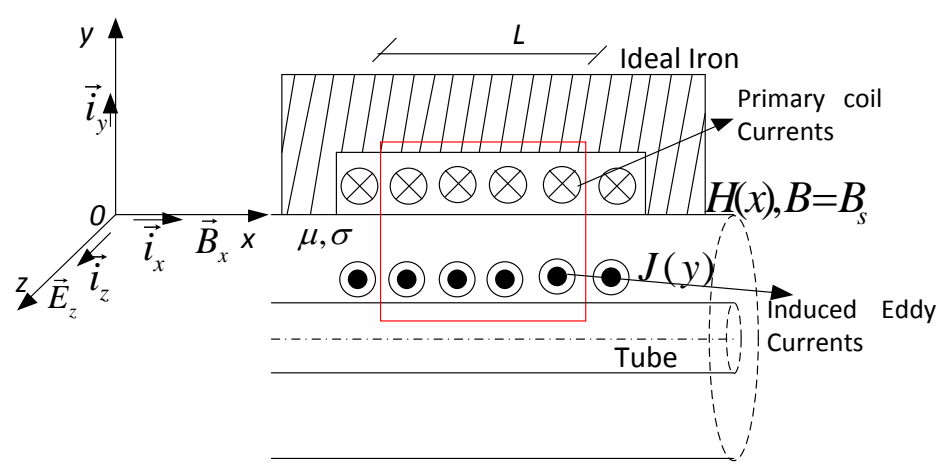

Figure 3.2: The penetration of $\mathrm{H}$, having the same tangential component just outside and inside the tube

\subsection{The Field Equations for Analytical Model}

Problems involving magnetic fields and current flow are formulated in terms of differential or integral equations. A differential equation describes the behaviour of magnetic field and some related quantity, at a point in terms of local source current density (if any). The equation is then solved within the region and the influence of sources outside the region is conveyed to the solution by the boundary conditions. Integral equations, on the other hand, express the field in terms of all the sources, the effect of the sources being summed to derive the resultant field. This would seem a better way to proceed than differential equation approach, but the fact integral equations are much more difficult to solve. This reason for this is not far-fetched, [40] the time rate of change of magnetic field produces additional sources (eddy currents), so that the field also appears in the integrand. The Eddy current analysis is essential in the determination of electric machine parameter [41], however it is also useful in part of the analysis of this thesis.

The basic field (Maxwell) equations if the displacement current (which is negligible in the conductor) is omitted are; 
$\nabla \times \vec{E}=-\frac{\partial \vec{B}}{\partial t} \quad($ Faraday's Law $)$

$\nabla \times \vec{H}=\vec{J} \quad$ (Ampere's Law)

$\nabla \cdot \vec{B}=0$

$\vec{J}=\sigma \vec{E} \quad($ Ohm's law)

Where $\vec{E}$ is the electric field strength, $\vec{H}$ the Magnetic field strength, $\vec{B}$ the magnetic flux density, and $\vec{J}$ the current density, $t$ the time and $\sigma$ is the conductivity. It is seen from equation 3.2 that in a conductor, the electric field strength is linked to the current density by Ohm's law

To complete the sets of equations 3.1-3.4 we add the constitutive equation relating $\vec{B}$ and $\vec{H}$, which is

$$
\vec{B}=\mu \vec{H}
$$

Where $\mu$ denotes the magnetic permeability which is the product of $\mu_{0} \mu_{r}$ (free space and relative permeability) for a linear material and for non-linear material, we can go through the references $[43,44]$

Inductive power transfer (IPT) via this metallic wall has limitations because of eddy currents and skin- depth of the material used, therefore in section 3.3 we will discuss about it as we proceed with our analysis

\subsection{The Eddy Currents and Skin Effect}

Eddy currents only flow close to the surface of a conductor (fig 3.2) and their depth of penetration depends upon the conductivity, the magnetic properties of the material and the frequency of excitation. The choice of rectangular coordinates would make this analysis less complicated compared to cylindrical coordinates, therefore, B-field, J-field and E- field components are written in rectangular coordinate system from fig 3.2 as

$$
\begin{aligned}
\vec{B} & =\left[\begin{array}{l}
B_{x} \\
B_{y} \\
B_{z}
\end{array}\right] \\
\vec{E} & =\left[\begin{array}{l}
E_{x} \\
E_{y} \\
E_{z}
\end{array}\right] \\
\vec{J} & =\left[\begin{array}{l}
J_{x} \\
J_{y} \\
J_{z}
\end{array}\right]
\end{aligned}
$$


In the fig. 3.2, the x-component of magnetic flux density which is constrained to be a spatial function of $y$, so it is assumed to be in $x$-direction and independent of z-direction. It also varies sinusoidally with time. As a result, the electric field and eddy currents are induced entirely in zdirection, therefore for further analysis equation 3.6 will now be written as

$$
\begin{aligned}
\vec{B} & =\left[\begin{array}{c}
B_{x}(y) \\
0 \\
0
\end{array}\right] \\
\vec{E} & =\left[\begin{array}{c}
0 \\
0 \\
E_{z}(y)
\end{array}\right] \\
\vec{J} & =\left[\begin{array}{c}
0 \\
0 \\
J_{z}(y)
\end{array}\right]
\end{aligned}
$$

From vector calculus for Cartesian coordinates, if we use equation 3.5 in equation 3.2 , then equation s 3.1 and 3.2 produces

$$
\begin{gathered}
\nabla \times \vec{B}=\left|\begin{array}{ccc}
\vec{i}_{x} & \vec{i}_{y} & \vec{i}_{z} \\
\frac{\partial}{\partial x} & \frac{\partial}{\partial y} & \frac{\partial}{\partial z} \\
B_{x} & B_{y} & B_{z}
\end{array}\right|=\left[\begin{array}{c}
0 \\
0 \\
-\frac{\partial B_{x}}{\partial y}
\end{array}\right]=\left[\begin{array}{c}
0 \\
0 \\
\mu J_{z}
\end{array}\right] \\
\nabla \times \vec{E}=\left|\begin{array}{ccc}
\vec{i}_{x} & \vec{i}_{y} & \vec{i}_{z} \\
\frac{\partial}{\partial x} & \frac{\partial}{\partial y} & \frac{\partial}{\partial z} \\
E_{x} & E_{y} & E_{z}
\end{array}\right|=\left[\begin{array}{c}
\frac{\partial E_{z}}{\partial y} \\
0 \\
0
\end{array}\right]=\left[\begin{array}{c}
-\frac{\partial B_{x}}{\partial t} \\
0 \\
0
\end{array}\right]
\end{gathered}
$$

where $\vec{i}_{x}, \vec{i}_{y}$, and $\vec{i}_{z}$ are unit vectors for $\mathrm{x}-, \mathrm{y}-$, and $\mathrm{z}$-axes respectively.

Thus, using equation 3.4 , equation 3.9 becomes

$\frac{\partial E_{z}}{\partial y}=\frac{1}{\sigma} \frac{\partial J_{z}}{\partial y}=-\frac{\partial B_{x}}{\partial t}$

therefore,

$$
\frac{\partial J_{z}}{\partial y}=-\sigma \frac{\partial B_{x}}{\partial t}
$$

Differentiating equation 3.8 with respect to $y$ and including it in equation 3.11 , we obtain 


$$
\begin{aligned}
& \frac{\partial^{2} B_{x}}{\partial y^{2}}=-\mu \frac{\partial J_{z}}{\partial y}=\sigma \mu \frac{\partial B_{x}}{\partial t} \\
& \frac{\partial^{2} B_{x}}{\partial y^{2}}=\sigma \mu \frac{\partial B_{x}}{\partial t}
\end{aligned}
$$

Therefore, we need to find the solutions to equations 3.11 and equations 3.12 so as to estimate the eddy current losses. In order to find the efficiency of power transfer we need to evaluate the field.

\subsection{The Magnetic field and Current Density Solutions}

Since the excitation is sinusoidal and hence periodic in time, we can use phasor notation. The time variation is eliminated and the quantities become complex and dependent on space only. Phasors are used in engineering to simply computations involving sinusoids, where they can often reduce a differential equation to an algebraic one. However in this case, we can eliminate the time variations of $B$ to just space variations by using $e^{j \omega t}$ format.

Let say,

$B_{x}=\hat{B} \cos (\omega t+\varphi)$

where,

$B_{x}$ is the $x$ component of $B$ (magnetic flux density) that is varying with time

$\varphi$ is a constant (in radians) known as the phase or phase angle of the sinusoid

$\hat{B}$ is a constant known as the amplitude of the sinusoid. It is the peak value of the function.

$\omega$ is the angular frequency given by $\omega=2 \pi f$ where $f$ is frequency.

$t$ is time.

Equation 3.13 can be expressed as

$$
\begin{aligned}
& B_{x}=\operatorname{Re}\{\hat{B}(\cos (\omega t+\varphi)+j \sin (\omega t+\varphi))\} \\
& =\operatorname{Re}\left\{\hat{B} e^{j \varphi} e^{j \omega t}\right\}=\operatorname{Re}\left\{\underline{B} e^{j \omega t}\right\}
\end{aligned}
$$

Where $\underline{B}$ is the combination of $\hat{B}$ and $e^{j \varphi}$

Thus, if we differentiate equation 3.14 we have

$$
\frac{\partial B_{x}}{\partial t}=\operatorname{Re}\left\{\frac{d}{d t} \underline{B} e^{j \omega t}\right\}=\operatorname{Re}\left\{j \omega \underline{B} e^{j \omega t}\right\}
$$

Therefore, a sinusoidal time variation of the field quantities enables us to replace $j \omega$ for $\partial / \partial t$ and the components of $\underline{B}$ and $\underline{J}$ are now complex that could be solved in one-dimension. Thus using equ 3.15 , eqn 3.12 becomes 


$$
\frac{\partial^{2} \underline{B}}{\partial y^{2}}=-\mu \frac{\partial \underline{J}}{\partial y}=j \omega \sigma \mu \underline{B}
$$

therefore, we can write a second order homogeneous differential equation in equation 3.12 as

$$
\frac{\partial^{2} \underline{B}}{\partial y^{2}}-j \omega \sigma \mu \underline{B}=0
$$

This is a second order homogenous differential equation with constant coefficients and the general solution is

$$
\underline{B}=\underline{C}_{1} e^{(\sqrt{j \omega \sigma \mu} \cdot y)}+\underline{C}_{2} e^{(-\sqrt{j \omega \sigma \mu} \cdot y)}
$$

This may be checked by substituting into equation 3.17 and it is more convenient to define the skin depth

$$
\delta=\sqrt{2 /(\omega \mu \sigma)}
$$

So that equation 3.18 becomes,

$$
\underline{B}=\underline{C}_{1} e^{(\sqrt{2 j} \cdot y / \delta)}+\underline{C}_{2} e^{(-\sqrt{2 j} \cdot y / \delta)}
$$

The $\sqrt{j}$ in equation 3.20 can be eliminated by noting that

$$
\sqrt{j}=e^{j(\pi / 4)}=\frac{1+j}{\sqrt{2}}
$$

Then,

$$
\underline{B}=\underline{C}_{1} e^{\left((1+j)^{y} / \delta\right)}+\underline{C}_{2} e^{\left(-(1+j)^{y} / \delta\right)}
$$

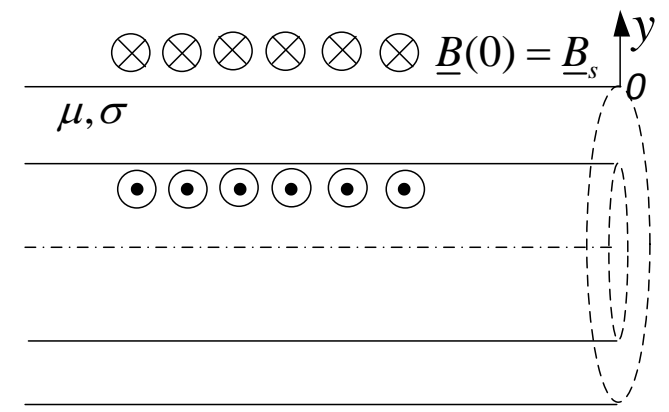

Figure 3.3: Magnetic flux density variations magnitude with skin depth

From a physical standing point $C_{1}$ should be zero since the value of magnetic field seems to be reducing as we go from the surface of the wall to inside. Otherwise the first term would go to infinity as y approached infinity. This can be envisaged by looking at figure 3.3. Therefore, if we call the flux density at the surface $\underline{B}(0)=\underline{B}_{s}$ then equation 3.22 becomes

$$
\underline{B}=\underline{B}_{s} e^{\left(-(1+j)^{y} / \delta\right)}=\underline{B}_{s} e^{(-y / \delta)} e^{\left(-j^{y} / \delta\right)}
$$


If we consider equation 3.15, the instantaneous value of the magnetic flux density in equation 3.23 is given by

$$
\begin{aligned}
& B_{x}=\operatorname{Re}\left\{\underline{B}_{s} e^{(-y / \delta} e^{\left(-j^{y} / \delta\right)} e^{j \omega t}\right\} \\
& B_{x}=B(y, t)=B_{s} e^{(-y / \delta)} \cos (\omega t-y / \delta)
\end{aligned}
$$

$B_{s}$ is the magnetic flux at the surface and its analysis is discussed in section 3.5 , but $\underline{B}$ is a complex quantity that is made of the product of two exponential terms (equation 3.23). The first term indicates that the magnitude of the flux density is decreasing exponentially with $y$. At depth $y=\delta$ the flux is $1 / e$ of its value at the surface (i.e the value of $\underline{B}$ is $37 \%$ of $\underline{B}_{s}$ ), see figure 3.4. The second exponential term has a magnitude of one and describes the phase shift of the flux density. At a depth $y=\delta$, the flux density lags the surface flux density $B_{s}$ by one radian. The parameter $\delta$ as earlier defined in equation 3.17 is the depth of penetration and it depends on the material properties ( $\sigma$ and $\mu$ ) and on frequency, not on flux density or the current.

Appendix A.1 gives the matlab scripts that generate the figures 3.4 and 3.5 respectively using equations 3.21 and 3.23. This is done for a particular material (stainless steel) with conductivity of $1.35 \mathrm{MS} / \mathrm{m}$ and relative permeability of 1 at frequency of $100 \mathrm{~Hz}$ when current of $10 \mathrm{~A}$ flows in the primary winding

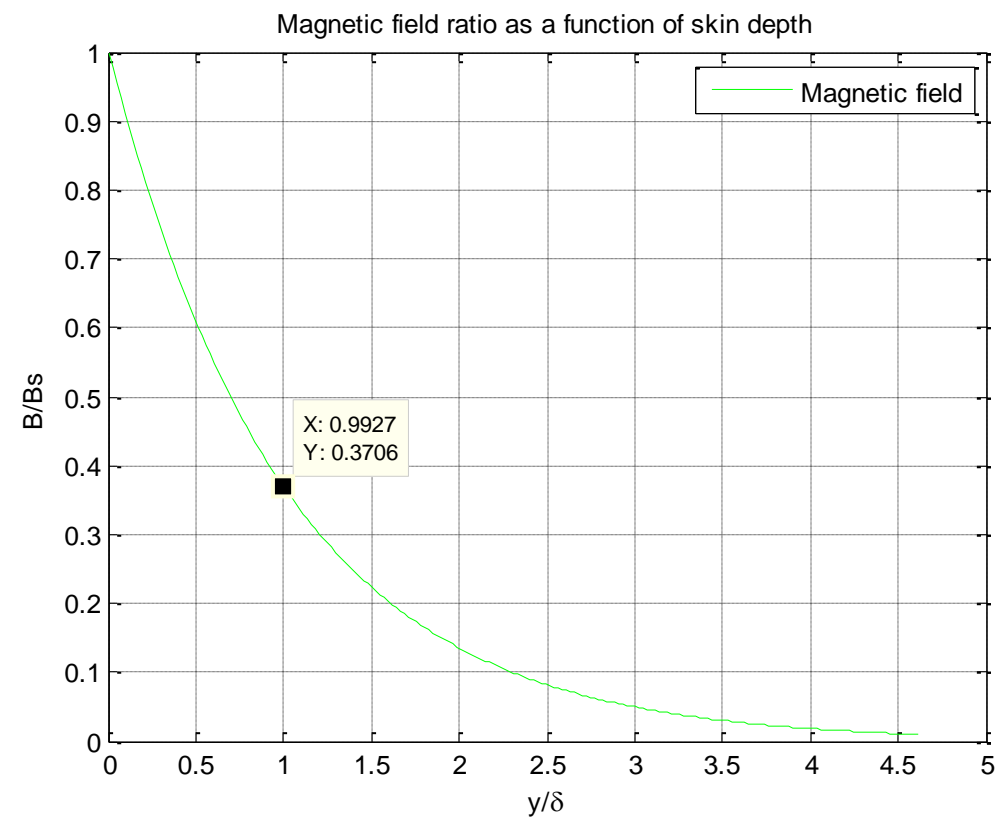

Figure 3.4: Magnetic field as a function of skin depth

The expression for the complex current density can be obtained if we consider equation 3.8 and this is found by differentiating the complex flux density expression in equation 3.23 , thus, 


$$
\underline{J}=\frac{1}{\mu} \frac{\partial \underline{B}}{\partial y}=-\frac{\underline{B}_{s}}{\mu} \frac{(1+j)}{\delta} e^{\left(-(1+j)^{y} / \delta\right)}
$$

If we apply equation 3.21 to the above equation we have,

$$
\begin{aligned}
& \underline{J}=-\frac{\underline{B}_{s}}{\mu} \frac{\sqrt{2}}{\delta} e^{j(\pi / 4)} e^{\left(-(1+j)^{y} / \delta\right)} \\
& =-\frac{\underline{B}_{s}}{\mu} \frac{\sqrt{2}}{\delta} e^{\left(-\frac{y}{\delta}\right)} e^{\left(-j^{y} / \delta\right)} e^{j(\pi / 4)}
\end{aligned}
$$

We can write $\underline{J}_{s}=-\frac{\underline{B}_{s}}{\mu} \frac{\sqrt{2}}{\delta}$ to make equation 3.26 looks like equation 3.23 , therefore, equation 3.26 becomes

$$
\underline{J}=\underline{J}_{s} e^{(-y / \delta} e^{-j\left(y / \delta^{-\pi / 4}\right)}
$$

Where $J_{s}$ is current density at the surface and using this equation, it produces Fig 3.5 which similar interpretation as in fig 3.4

Analogous to equation 3.24 the instantaneous value of the Current density in equation 3.23 is given by

$$
\begin{aligned}
& J_{z}=\operatorname{Re}\left\{\underline{J}_{s} e^{(-y / \delta)} e^{-j\left(y / \delta^{-\pi / 4}\right)} e^{j \omega t}\right\} \\
& J_{z}=\underline{J}_{s} e^{(-y / \delta)} \cos (\omega t-(y / \delta-\pi / 4))
\end{aligned}
$$

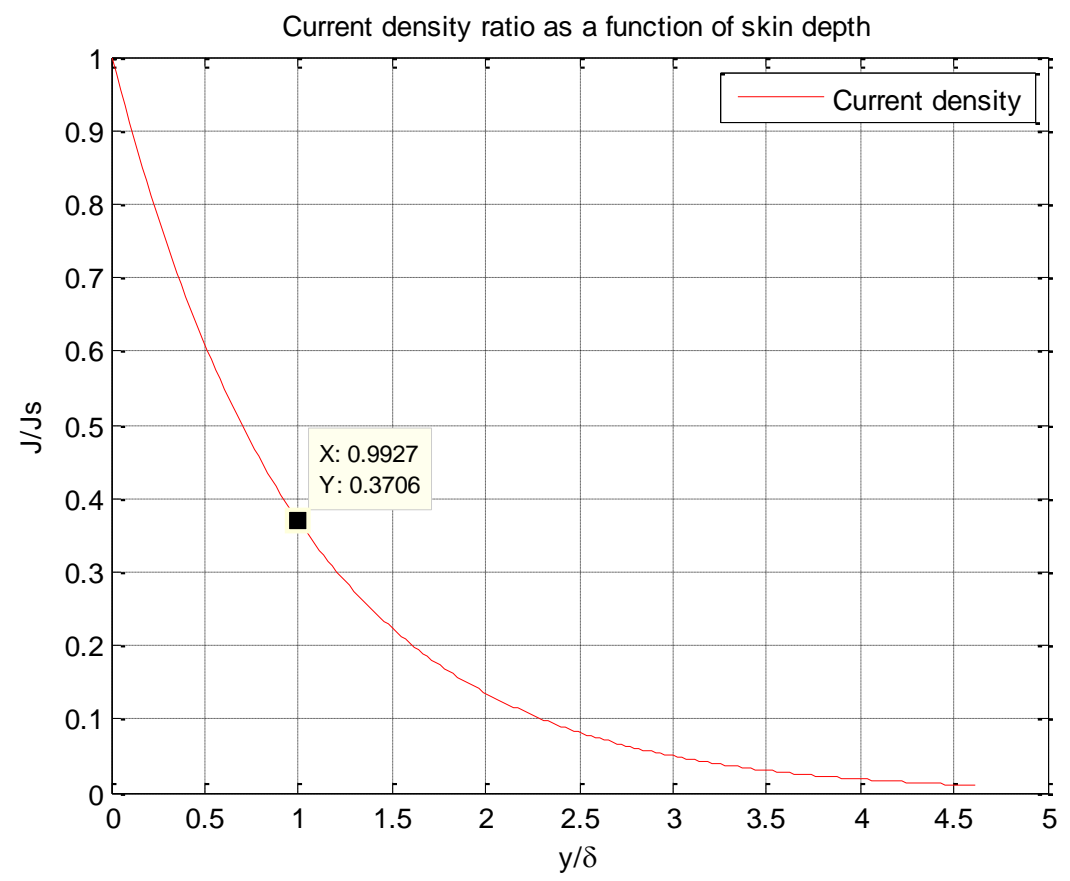

Figure 3.5: Current density as a function of skin depth 
It is also important to know the magnetic flux density ratio at the surface to the other side of the pipe's wall. Therefore we will look into that in section 3.5 for us to see how things change in the configuration adopted.

\subsection{The Magnetic flux density Ratio}

It is also essential to have a quick view of ratio of magnetic flux density to have the idea about percentage of magnetic field and magnetic power ratio. Therefore, let us discuss at the analysis of Magnetic flux inside a metal pipe that is just below the primary winding outside (i.e at the surface of) the pipe as shown in fig 3.6. Later we shall talk about the ratio.

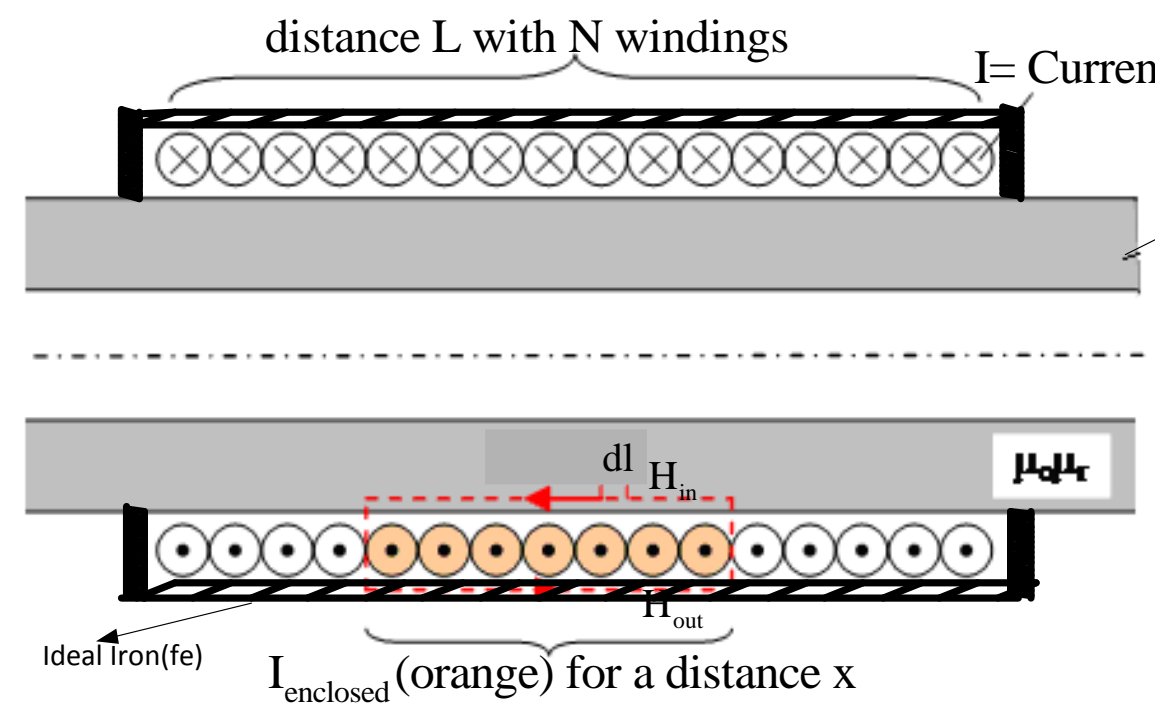

Figure 3.6: Metal pipe with primary coil aligned and concentric

The analysis of figure 3.6 becomes much simple if we assume a perfect iron outside the coil. This can lead to discrepancies in actual measurements due to such simplification. Therefore, if Ampere's law is applied to the red dotted line in fig 3.5, we have

$$
\begin{aligned}
& \oint H \cdot d l=I_{\text {enclosed }} \\
& \int_{x} H_{\text {in }} \cdot d l-\int_{x} H_{\text {out }} \cdot d l=\left(\frac{N}{L} \cdot x\right) \cdot I \\
& H_{\text {in }} \cdot x-H_{\text {out }} \cdot x=\left(\frac{N}{L} \cdot x\right) \cdot I \\
& \frac{B_{\text {in }}}{\mu_{o} \mu_{r}}-\frac{B_{\text {out }}}{\mu_{o} \mu_{r(f e)}}=\left(\frac{N}{L}\right) \cdot I
\end{aligned}
$$


We assumed an idea iron on outer part of the coil, therefore $\mu_{r(f e)} \rightarrow \infty$ and thus

$$
\frac{B_{i n}}{\mu_{o} \mu_{r}}=\left(\frac{N}{L}\right) . I
$$

Then

$$
B_{i n}=\mu_{o} \mu_{r}\left(\frac{N}{L}\right) . I
$$

Therefore equation 3.34 can be used to determine the magnetic field inside the ferro metal core coil with $\mu_{\mathrm{r}}>1$, but for stainless steel, $\mu_{\mathrm{r}}=1$, thus

$$
B_{\text {in }}=\mu_{o}\left(\frac{N}{L}\right) . I
$$

$B_{\text {in }}$ is similar to the absolute value of the magnetic flux $\underline{B}_{s}$ at the surface of the metal wall as used in section 3.4 as well as figure 3.2.

We shall now discuss the ratio of magnetic flux density at the surface to the one inside the wall of the pipe. This ratio is very vital because power is a function of square of amplitude of magnetic (B) flux density in a material ( $P \alpha B^{2}$ ) according to [40], [45], [46], and [47] therefore, finding the ratio of the magnetic (B) magnetic flux density (equation 3.24) to the magnetic (B) flux density at the surface of the wall of the tube to will give an indication of power ratio. at

$$
y=0, \quad B(0, t)=B_{s}
$$

and at

$$
y=h, \quad B(h, t)=B_{s} e^{(-h / \delta)}
$$

where (where $h$, is the thickness of the tube)

Therefore, the magnetic density ratio is

$$
\frac{|B|}{B_{s}}=e^{(-h / \delta)}
$$

An important parameter for efficiency could be determined by an assumption that the power is a function of square of the magnetic flux density while other quantities are rather not changing. Therefore, we can say the input power is a function of absolute value of surface magnetic flux density,

So, at

$y=0, \quad P_{i n}=P(0) \alpha\left\{B_{s}^{2}\right\}$

and 


$$
y=h, \quad P(h) \alpha\left\{B_{s} e^{(-h / \delta)}\right\}^{2}
$$

Where, $P_{i n}$ which assumed to be input power is a function of square of surface magnetic flux and $P(h)$ is assumed to be the power at the inner surface since $h$ is the thickness of the wall (figure 3.7). The efficiency can be expressed in term of magnetic flux density following our assumption that other quantities are not changing, but before we define efficiency a load (resistor R) has to be connected to the secondary coil to so the an the output power can be determined as in fig 3.6.

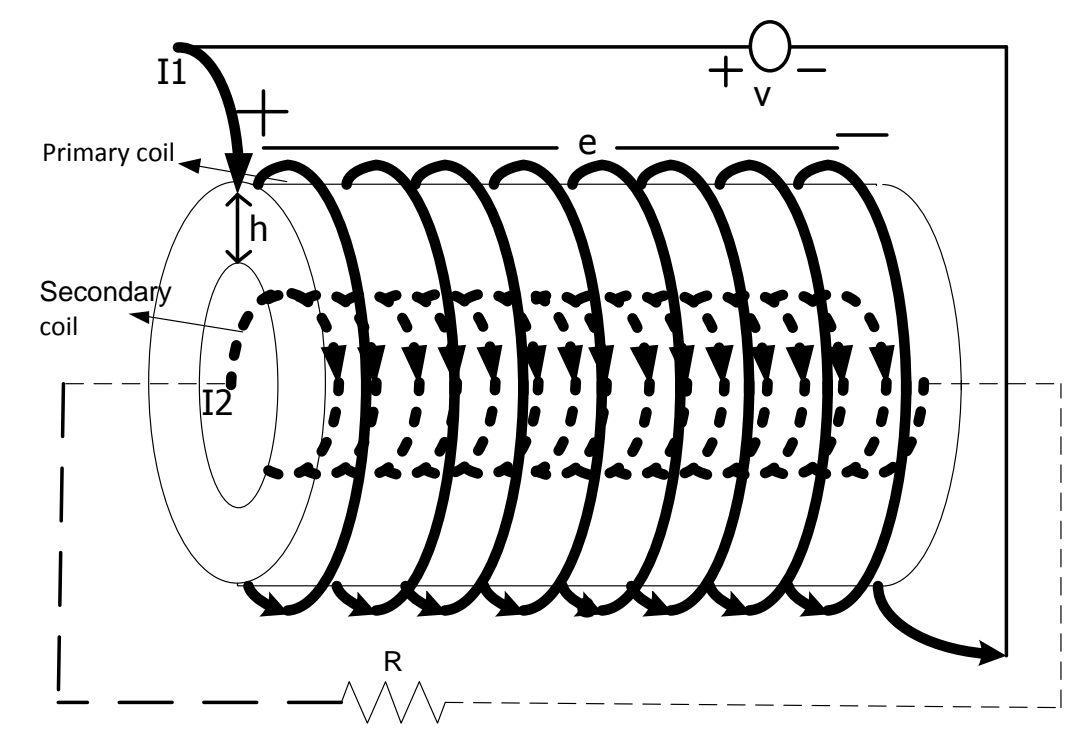

Figure 3.7: Metal pipe wall with primary coil connected to power source and Secondary to coil Load

From equation 3.34, the approximate equivalent absolute value of $\underline{B}_{s}$ in term of number of turns per length $N_{1} / l_{1}$ and current $I_{1}$ flowing through the primary coil for a material like stainless steel is

$$
B_{s}=\mu_{o} \mu_{r}\left(\frac{N}{L}\right) . I
$$

Thus, at $t=0$, we can express a factor, $\gamma$ that would be useful for future analysis in magnetic power ratio as,

$$
\gamma=\frac{P(h)}{P_{i n}}=\frac{B_{s}^{2} e^{(-2 h / \delta)}}{B_{s}^{2}}=e^{(-2 h / \delta)}
$$

If we substitute the expression of the skin depth $\delta$ obtain in equation 3.19 in equation 3.43 , it becomes 


$$
\gamma=e^{(-2 h / \delta)}=e^{(-2 h \sqrt{\pi f \mu \sigma})}
$$

The equation 3.44 will be used to in the experimental model to check the power ratio in with the given thickness of the tube.

It is good to have a possibility of making an equivalent circuit model out of figure 3.7. This will provide another approach to analytical model, but the determination of the parameters in the model will be left for future investigation.

\subsection{Equivalent Circuit Model}

It is important to make an equivalent circuit model for figure 3.7 to simplify our analysis. This is shown in figure 3.8

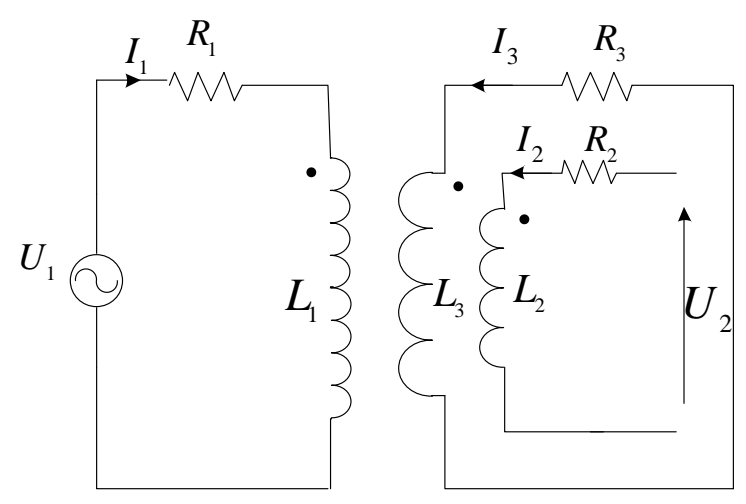

Figure3.8: Equivalent model of figure 3.7 without load

If we write voltage equations for the above circuit we have,

$$
\begin{aligned}
& U_{1}=R_{1} i_{1}+\frac{d \lambda_{1}}{d t} \\
& U_{2}=R_{2} i_{2}+\frac{d \lambda_{2}}{d t} \\
& 0=R_{3} i_{3}+\frac{d \lambda_{3}}{d t}
\end{aligned}
$$

Where $\lambda_{1}, \lambda_{2}$ and $\lambda_{3}$ is the flux linkage in inductor $L_{1}, L_{2}$ and $L_{3}$ respectively and they are espressed as

$$
\begin{aligned}
& \lambda_{1}=L_{1} i_{1}+M_{12} i_{2}+M_{13} i_{3} \\
& \lambda_{2}=M_{21} i_{1}+L_{2} i_{2}+M_{23} i_{3} \\
& \lambda_{3}=M_{31} i_{1}+M_{32} i_{2}+L_{3} i_{3}
\end{aligned}
$$


With $M_{x y}=M_{y x}$ representing the mutual inductance, therefore using equation 3.46 in 4.45, we have

$U_{1}=R_{1} i_{1}+L_{1} \frac{d i_{1}}{d t}+M_{12} \frac{d i_{2}}{d t}+M_{13} \frac{d i_{3}}{d t}$

$U_{2}=M_{12} \frac{d i_{1}}{d t}+R_{2} i_{2}+L_{2} \frac{d i_{2}}{d t}+M_{23} \frac{d i_{3}}{d t}$

$0=M_{13} \frac{d i_{1}}{d t}+M_{23} \frac{d i_{2}}{d t}+R_{3} i_{3}+L_{3} \frac{d i_{3}}{d t}$

Using a phasor notation, equation 3.47 becomes

$U_{1}=R_{1} I_{1}+j \omega L_{1} I_{1}+j \omega M_{12} I_{2}+j \omega M_{13} I_{3}$

$U_{2}=j \omega M_{12} I_{1}+R_{2} I_{2}+j \omega L_{2} I_{2}+j \omega M_{23} I_{3}$

$0=j \omega M_{13} I_{1}+j \omega M_{23} I_{2}+R_{3} I_{3}+j \omega L_{3} I_{3}$

We can write a matrix model for equation 3.48 , thus

$\left[\begin{array}{c}U_{1} \\ U_{2} \\ 0\end{array}\right]=\left[\begin{array}{ccc}\left(R_{1}+j \omega L_{1}\right) & j \omega M_{12} & j \omega M_{13} \\ j \omega M_{12} & \left(R_{2}+j \omega L_{2}\right) & j \omega M_{23} \\ j \omega M_{13} & j \omega M_{23} & \left(R_{3}+j \omega L_{3}\right)\end{array}\right] \cdot\left[\begin{array}{c}I_{1} \\ I_{2} \\ I_{3}\end{array}\right]$

The determination of the constants in the model not included in this thesis, however this can be investigated for future analysis. 


\section{Chapter 4}

\section{Experimental Analysis and Discussion of Results}

\subsection{Introduction}

It is important to validate the analytical model made in chapter 3 , therefore experiments will be an interesting way that will be helpful in achieving this goal. The analytical model results predicted will be checked experimentally if it is really possible to transfer power and the efficiency of the power transfer. In this experimental analysis, stainless steel pipe will be used as wall between the primary and secondary coils due to its low permeability. We shall now discuss materials for the experimental set up in section 4.1

\subsection{The experimental set-up}

The choice of materials to be used are according to the conventional rated value (standard manufacturer specification from the data sheet), therefore little analytical finds are done where necessary. The following are the essential equipment used in the experiment.

1. Amplifier of (+/-75V max) and (+/-5A max) outputs

2. Copper wire of $1.25 \mathrm{~mm}$ diameter

3. Stainless steel (cylindrical) pipe of about $220 \mathrm{~mm}$ diameter and $8 \mathrm{~mm}$ thickness

4. Measuring instruments (Digital Power Meters, Digital Multimeters, Digital automatic RLC Meter)

5. Load

6. Oscilloscope

Figure 4.1a shows the preliminary experimental set-up for the coils and the pipe while figure 4.1b describes the cross-sectional view

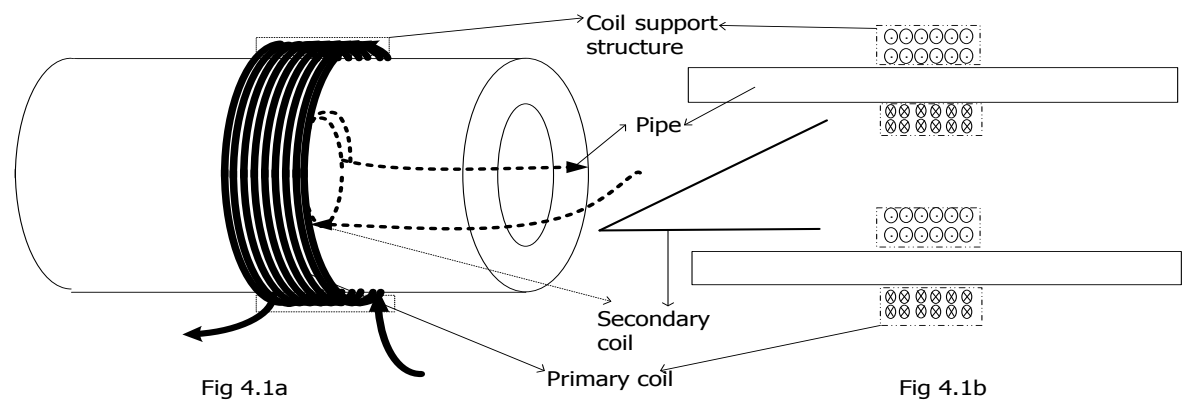

Figure 4.1: (a) Preliminary experiment set up showing the coils (Primary and Secondary) on the pipe, (b)Cross-sectional view of figure 4.1 a 


\subsubsection{Amplifier}

The amplifier used is a Current/ Voltage source bipolar operational power amplifier with current output (+/- 5A max) and Voltage output (+/-75 max) with frequency range of $(0-20 \mathrm{kHz})$. This stands as the source of power supply to the primary coil. Figure 4.2 shows the setup arrangements of the amplifier

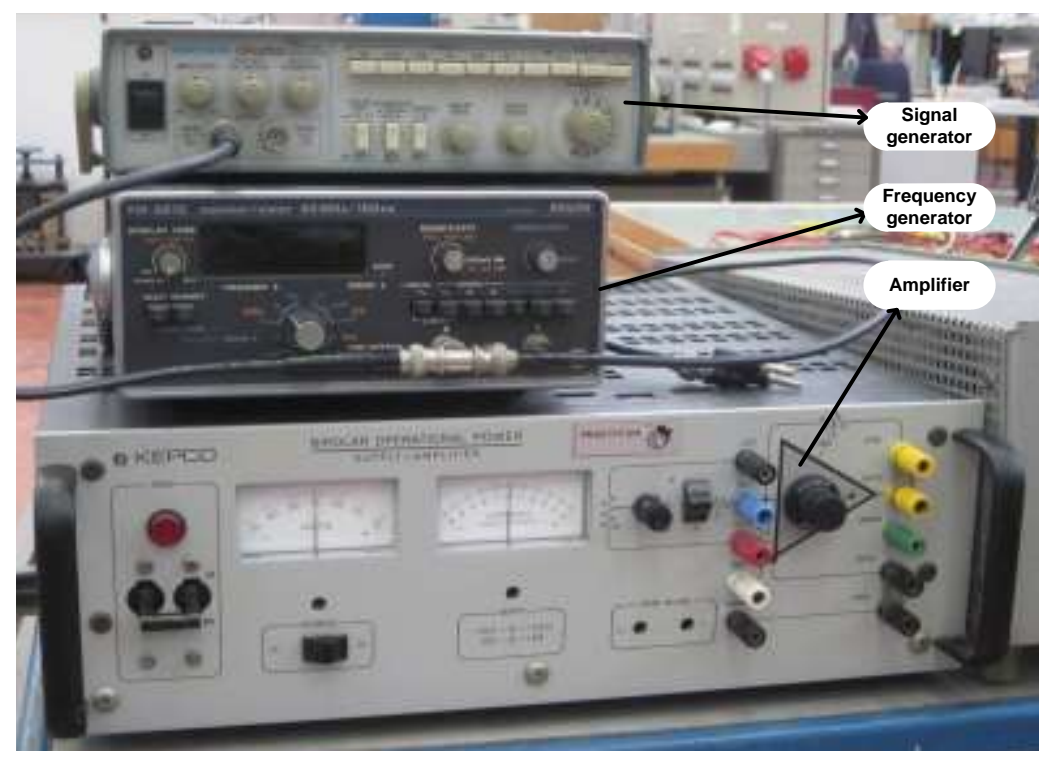

Figure 4.2: Amplifier (+/-75V, +/-5A, 0-20kHz) set-up

\subsubsection{Copper Wire}

An enamelled copper wire of $1.25 \mathrm{~mm}$ diameter is used to make both primary and secondary coils. Enamelled wire is a wire coated with a thin layer of insulation to prevent the wire surfaces from being in a short circuit when wound into a coil. The Figure 4.3 shows a sample of the copper wire used to make the coils.

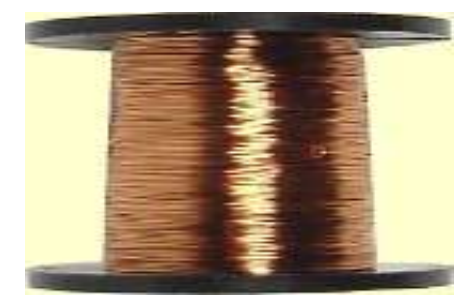

Figure 4.3: Sample of copper wire used to make the coils

\subsubsection{Stainless Steel Pipe}

The material properties according to the manufacturer are shown in table 4.1. However, a cylindrical pipe made of grade $316 \mathrm{~L}$ stainless steel [48] is used at start to make sure there is enough power transfer to the secondary coil. A ferro-steel of grade ST37 [49] is left out for future works. 
Table 4.1: Material parameters and calculated skin depth

\begin{tabular}{|c|c|c|c|c|c|c|c|c|c|c|c|}
\hline \multirow[t]{2}{*}{ Material } & \multirow[t]{2}{*}{ Grade } & \multirow[t]{2}{*}{$\begin{array}{c}\text { pipe } \\
\text { length } \\
{[\mathrm{m}]}\end{array}$} & \multirow[t]{2}{*}{$\begin{array}{c}\text { outside } \\
\text { diameter } \\
\text { [mm] }\end{array}$} & \multirow[t]{2}{*}{$\begin{array}{c}\text { wall } \\
\text { thickness } \\
{[\mathrm{mm}]}\end{array}$} & \multirow[t]{2}{*}{$\begin{array}{c}\text { pipe } \\
\text { weight } \\
\text { [kg] }\end{array}$} & \multirow[t]{2}{*}{$\begin{array}{c}\text { Electrical } \\
\text { cond. } \sigma \\
{[\mathrm{MS} / \mathrm{m}]}\end{array}$} & \multirow{2}{*}{$\begin{array}{c}\text { Rel. mag. } \\
\text { Permeability } \\
\text { pr [-] }\end{array}$} & \multicolumn{4}{|c|}{ calculated skindepth [mm] } \\
\hline & & & & & & & & at $40 \mathrm{H}_{2}$ & t $50 \mathrm{H}_{2}$ & at $70 \mathrm{~Hz}$ & at $100 \mathrm{~Hz}$ \\
\hline stainless steel & $316 \mathrm{~L}$ & 1 & 219.1 & 8.2 & 44 & 1.35 & $\sim 1$ & 68.49 & 61.26 & 51.77 & 43.32 \\
\hline ferro-steel & ST37 & 1 & 219.1 & 10 & 54 & 6.25 & $\sim 500$ & 1.42 & 1.27 & 1.08 & 0.9 \\
\hline
\end{tabular}

\subsubsection{Measuring Instruments}

The current, voltage, input and output power etc. are determined by the flowing measuring instruments.

- Digital Multimeter- to measure Currents, Voltage \{figure 4.4a\}

- Digital Power Wattmeter-to measure, power factor and both input and output Power \{figure $4.4 b\}$

- $\quad$ Digital automatic RLC Meter-to measure and check coil inductance \{figure 4.4c\}
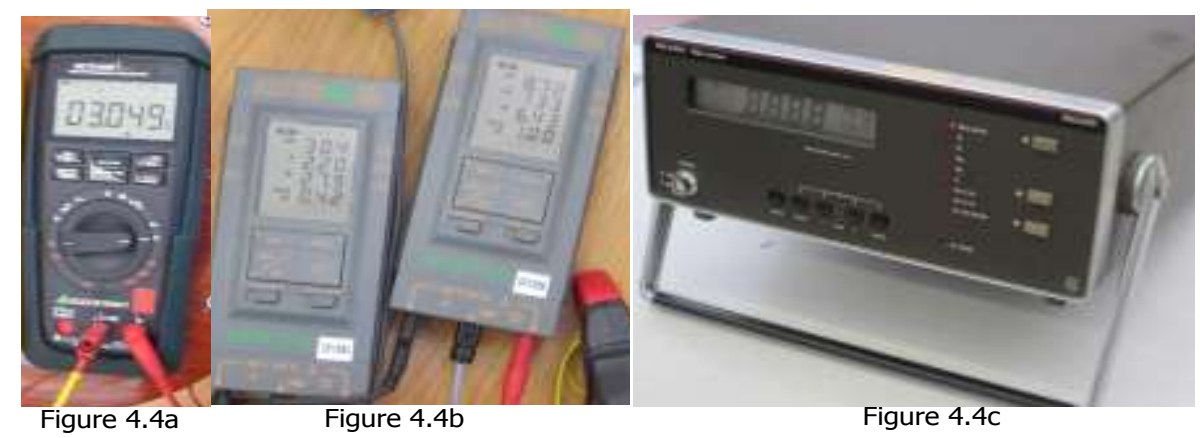

Figure 4.4: Measuring Meters (a) Multi-meter, (b) Power meter, (c) RLC meter

\subsubsection{Load}

Without a load connected to the secondary coil, there is no output power to be delivered; therefore the choice of the load connected to the secondary coil determines the quantity of output power to be measured. A variable resistor $(0-200 \mathrm{k} \Omega)$ is used as load to determine the quantity of output power delivered from the primary coil to the secondary coil.

\subsection{Experiment for IPT via a stainless steel pipe}

The experimental set-up for inductive power transfer (IPT) via a stainless steel pipe is shown in figure 4.5. The primary coil is wound outside the pipe and it is connected to the power supply amplifier of $(+/-$ $75 \mathrm{~V} \max )$ and (+/-5A max) outputs. A load resistance $R$ is connected to the secondary coil which is wound inside. 


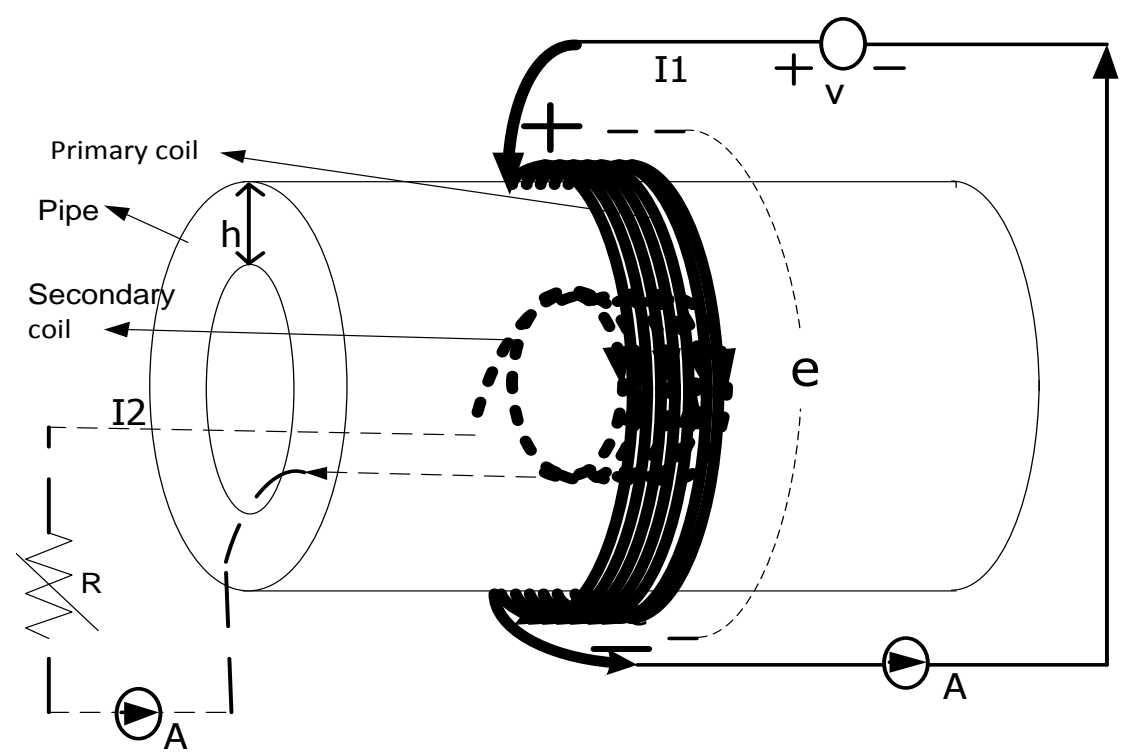

Figure 4.5: Experimental diagram

\subsubsection{Number of turns required and Inductance Calculations}

The maximum number of turns for primary coil to transfer a sufficient power to the secondary coil is required to be estimated based on the maximum output power of the amplifier used. Therefore, at a lower frequency of $100 \mathrm{~Hz}$, the inductance will be used to estimate the number of turns required in the primary coils. Figure 4.5 will be used to find the rough estimate of the number of turns by first determining the induced emf $e$ when current $I$ flows in the coil.

From fig 4.5 the applied voltage ( $v$ ) can be expressed as...

$v=R i+L \frac{d i}{d t} \approx L \frac{d i}{d t}$

Where $R$ the resistance of the coil is assumed to be negligible at high frequency, $L$ is the inductance of the coil and $i$ is the instantaneous current

It has been assumed that the waveforms of applied voltage and the resulting current are sinusoidal. Hence, the analysis can be greatly simplified by taking the instantaneous current to be

$i=I_{\max } \sin (\omega t)$

Therefore equation 4.1 becomes

$e=L \frac{d i}{d t}=\omega L I_{\max } \cos (\omega t)$ 
Where $I_{\max }$ is the maximum value of the current and $\omega=2 \pi f$, and $\mathrm{f}$ is the frequency in $\mathrm{Hz}$.

The maximum values of the available amplifier for current output (+/-5A max) /voltage output $(+/-75 \mathrm{~V}$ max $)$ source for the experiment should not be exceeded, so a maximum current of $\left(I_{\max }=5 \mathrm{~A}\right.$ ) and voltage $75 \mathrm{~V}$ will be used in the choice of inductance $(L)$ of at a frequency of $100 \mathrm{kHz}$.

Both primary and secondary coils are circular in shape and some certain numbers of turns are required for a meaningful voltage to be induced. According to [50] and [51], the inductance calculations in loop of wire with circular shapes in air as seen in figure 4.6 could be expressed as

$$
L \approx N^{2} \mathrm{R} \mu_{0} \mu_{r}\left(\ln \left(\frac{8 \mathrm{R}}{a}\right)-2\right)
$$

Where, $N$ is number of turns, $\mathrm{R}$ is the radius of the circle in $(\mathrm{m}), a$ is the radius of the wire in (m), $\mu_{0}$ is the permeability in a free medium $\left(\mu_{0}=4 \pi \cdot 10^{-7} \mathrm{H} / \mathrm{m}\right)$ and $\mu_{r}$ is relative permeability (for copper $\mu_{r}=1$ ).

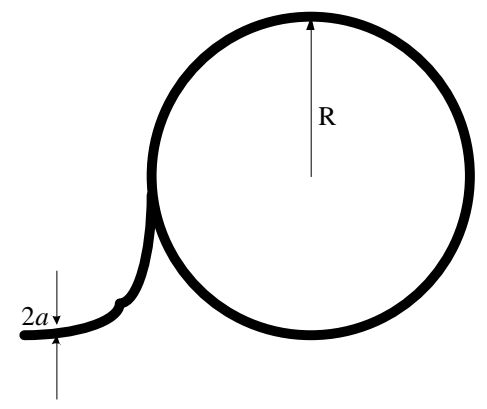

Figure 1 Figure 4.6: Loop of wire with circular shape

The inductance of the primary coil can be calculated from equation 4.3 so that the number of coil is determined from equation 4.4. Therefore, if we applied a voltage $75 \mathrm{~V}$ at a peak current of $5 \mathrm{~A}$ for a frequency of $100 \mathrm{kHz}$ in equation 4.3 , the inductance at time $(t=0)$ will be $23.87 \mathrm{mH}$ Using the value of this inductance $(23.87 \mathrm{mH})$ to determine the number of turns based on of primary coil of copper material $\left(\mu_{r}=1\right)$ from equation 4.4 , we have.

$$
N \approx \sqrt{\frac{L}{\mathrm{R} \mu\left(\ln \left(\frac{8 \mathrm{R}}{a}\right)-2\right)}}=\sqrt{\frac{L}{\mathrm{R} \mu_{0} \mu_{r}\left(\ln \left(\frac{8 \mathrm{R}}{a}\right)-2\right)}}
$$


A copper wire of diameter $1.25 \mathrm{~mm}$ is available to be used and can be used with the Current/Voltage source available for the experiment, therefore if wire radius ( $a=0.625 \mathrm{~mm})$ and coil radius ( $R=120 \mathrm{~mm}$ ) are substituted in the equation 4.5 .

Therefore, to the nearest whole number, $N \approx 172$ turns

It is expected that the number of turns in practice should be increased because the value of the inductance estimated in air will reduce when placed on the tube. This will lead to reduction in the primary voltage when place on tube. Therefore we need to increase the number of turns to compensate for the loss associated with the coil. Thus 320 turns is made for the primary coil. Secondary coil is also estimated like primary coil and the number is predicted to be 200 turns. However, the air gap between the coil and the pipe is very small due to the diameter $(185 \mathrm{~mm})$ of container holding the secondary coil. Therefore, 220 turns is made for the secondary coil.

\subsubsection{Resistance Calculations}

The resistance of a material (wire) is proportional to the length and inversely proportional to the area of the material. Therefore, the resistance of the coils and tube area where the coils are wound in figure 3.5 are determined. The general expression for resistance is

$$
R=\rho \frac{l}{A}
$$

where $\rho$ is the resistivity of the material whose resistance is to be determined. For primary coil and secondary coil $\rho$ is $1.68 \times 10^{-8} \Omega m$ and $7.41 \times 10^{-7} \Omega m$ for the stainless steel tube.

If we use the denotation used in figure 3.8, subscripts 1,2 and 3 refers to primary coil, secondary coil and the tube respectively.

For the primary coil, the number of turns $N_{1}=320$, coil diameter $D_{1}=240 \mathrm{~mm}$, wire radius $a_{1}=0.625 \mathrm{~mm}$, we have

$$
R_{1}=\rho_{1} \frac{l_{1}}{A_{1}}=\rho_{1} \frac{N_{1} \pi D_{1}}{\pi a_{1}^{2}}=3.30 \Omega
$$

Similarly, for the secondary coil, the number of turns $N_{2}=220$, coil diameter $D_{2}=185 \mathrm{~mm}$, wire radius $a_{2}=a_{1}=0.625 \mathrm{~mm}$, therefore we have 


$$
R_{2}=\rho_{2} \frac{l_{2}}{A_{2}}=\rho_{2} \frac{N_{2} \pi D_{2}}{\pi a_{2}^{2}}=1.75 \Omega
$$

In order to use the analytical model to estimate the eddy current losses, we find the resistance of the area of the pipe that is covered by the wire. Therefore figure 4.7 shows the section of the area covered by the coils. The length of area covered is $l_{t}=12.5 \mathrm{~cm} \approx 13 \mathrm{~cm}$, the thickness of the tube $h=7.6 \mathrm{~mm}$, the number of turn of tube is $N_{3}=1$ and the mean diameter of the tube $D_{3}=216 \mathrm{~mm}$, we have

$$
R_{3}=\rho_{3} \frac{l_{3}}{A_{3}}=\frac{N_{3} \pi D_{3}}{l_{t} h}=0.509 \mathrm{~m} \Omega
$$

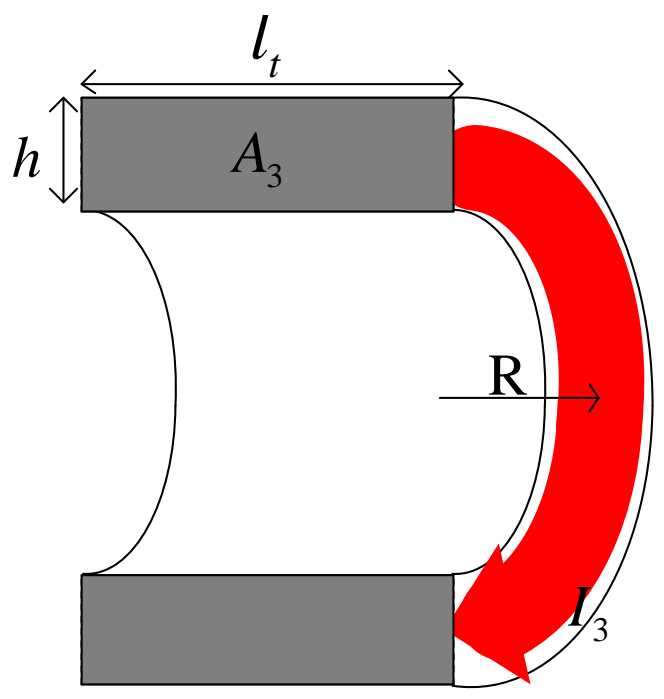

Figure 4.7: Tube section covered by the coils

This wall resistance is used to compare the analytical model with the experimental model using equation 3.28 in appendix A.2. We shall now look into the experimental results

\subsection{Measurements Results}

Using the information from the analytical results, we can see that frequency, conductivity, permeability etc. are important parameters, and therefore we shall do experiments by varying the load in the secondary coil to determine the optimum load (maximum efficiency) at different frequency, but focus more on $50 \mathrm{~Hz}$ because it will be easier and cheaper to operate at national grid frequency. It is also very vital to perform a "No-Load" and "Short-circuit" test to have the idea of the Nominal Voltage and Current respectively. We have not looked at the measurements made when we used different pipe materials as wall between the primary and the secondary coils. 


\subsubsection{Stainless steel measurement Results}

When stainless steel pipe is used as wall between primary and secondary coils we have some measurements which are recorded in different tables.

Resistance and inductances of the coils are first measured and there values in table 4.2 are very close to the calculated values in section 4.3.

Table 4.2: Measure value of Resistance and Inductance

\begin{tabular}{|c|c|c|c|c|c|c|c|}
\hline $\mathbf{R 1}$ in air & $\mathbf{L 1}$ in air & $\mathbf{R 1}$ in tube & $\mathbf{L 1}$ in tube & $\mathbf{R 2}$ in air & L2in air & $\mathbf{R 2}$ in tube & L2 in tube \\
\hline $3.52 \Omega$ & $29.69 \mathrm{mH}$ & $3.52 \Omega$ & $13.83 \mathrm{mH}$ & $1.93 \Omega$ & $9.43 \mathrm{mH}$ & $1.93 \Omega$ & $3.62 \mathrm{mH}$ \\
\hline
\end{tabular}

The no-load and short circuit tests are done only at $50 \mathrm{~Hz}$ and we can see the measurement in table 4.3. The first set of other measurements made at four different frequencies while varying the load resistance. Tables 4.4 to 4.7 show the measurement results and figures 4.8 to 4.11 shows the plots made from each table. The losses in each coil are is the product of square of currents flowing in the coil and the resistance of the coil.

Table 4.3: Short-circuit and no-load test at $50 \mathrm{~Hz}$

\begin{tabular}{|c|c|c|c|c|c|c|c|c|c|}
\hline Test & freq[Hz] & U1[V] & I1[A] & pf1 & P1[W] & U2[V] & I2[A] & pf2 & P2[W] \\
\hline Short circuit test & 50 & 30.7 & 3.54 & 0.63 & 68.47 & 0 & 3.08 & 0 & 0 \\
\hline No load Test & 50 & 30.8 & 2.97 & 0.42 & 38.42 & 9.68 & 0 & 0 & 0 \\
\hline
\end{tabular}

Table 4.4: varying Load $(R)$ at frequency of $(40 \mathrm{~Hz})$

\begin{tabular}{|c|c|c|c|c|c|c|c|c|c|c|c|c|c|}
\hline $\operatorname{RL}($ Meaured) $[\Omega]$ & $\mathrm{U} 1[\mathrm{~V}]$ & $\mathrm{II}[\mathrm{A}]$ & pf1 & $\mathrm{P} 1[\mathrm{~W}]$ & U2[V] & 12[A] & pf2 & $\mathrm{P} 2[\mathrm{~W}]$ & eff $(40 \mathrm{~Hz})$ & $\operatorname{Ploss}(\mathrm{T})[\mathrm{W}]$ & $\operatorname{Ploss}(\mathrm{P})[\mathrm{W}]$ & Ploss $(\mathrm{S})[\mathrm{W}]$ & ] Ploss(wall)[W] \\
\hline 2.08 & 30.4 & 3.59 & 0.62 & 67.66 & 4.00 & 1.921 & 1.00 & 7.68 & 11.36 & 59.98 & 45.37 & 7.12 & 7.49 \\
\hline 2.55 & 30.5 & 3.65 & 0.60 & 66.80 & 4.43 & 1.738 & 1.00 & 7.70 & 11.53 & 59.10 & 46.90 & 5.83 & 6.37 \\
\hline 3.80 & 30.3 & 3.59 & 0.57 & 62.00 & 5.33 & 1.404 & 1.00 & 7.48 & 12.07 & 54.52 & 45.37 & 3.80 & 5.35 \\
\hline 5.55 & 30.5 & 3.59 & 0.56 & 61.32 & 6.32 & 1.139 & 1.00 & 7.20 & 11.74 & 54.12 & 45.37 & 2.50 & 6.25 \\
\hline 7.52 & 30.5 & 3.56 & 0.54 & 58.63 & 6.90 & 0.918 & 1.00 & 6.33 & 10.80 & 52.30 & 44.61 & 1.63 & 6.06 \\
\hline 11.30 & 30.2 & 3.47 & 0.51 & 53.44 & 7.52 & 0.665 & 1.00 & 5.00 & 9.36 & 48.44 & 42.38 & 0.85 & 5.21 \\
\hline 18.00 & 30.3 & 3.49 & 0.51 & 53.93 & 8.12 & 0.451 & 1.00 & 3.66 & 6.79 & 50.27 & 42.87 & 0.39 & 7.00 \\
\hline 23.96 & 30.6 & 3.53 & 0.50 & 54.01 & 8.43 & 0.352 & 1.00 & 2.97 & 5.49 & 51.04 & 43.86 & 0.24 & 6.94 \\
\hline 29.57 & 30.3 & 3.49 & 0.49 & 51.82 & 8.51 & 0.288 & 1.00 & 2.45 & 4.73 & 49.37 & 42.87 & 0.16 & 6.33 \\
\hline
\end{tabular}




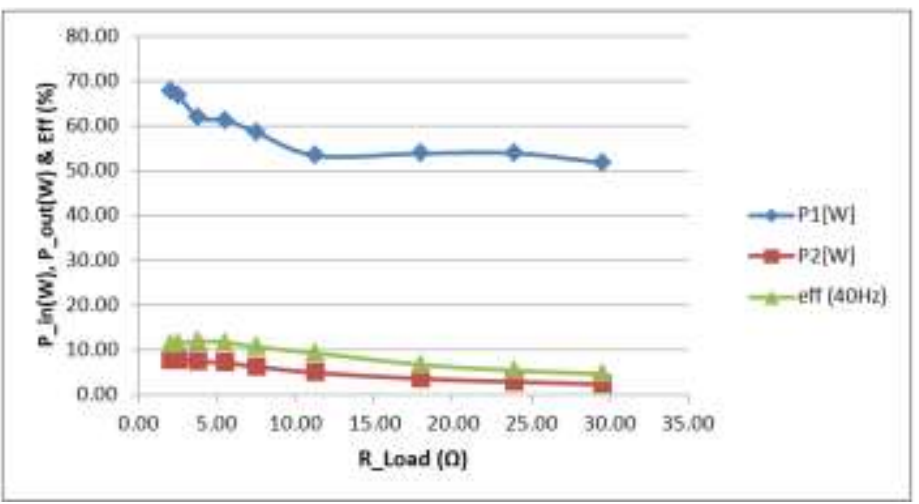

figure 4.8 (a)

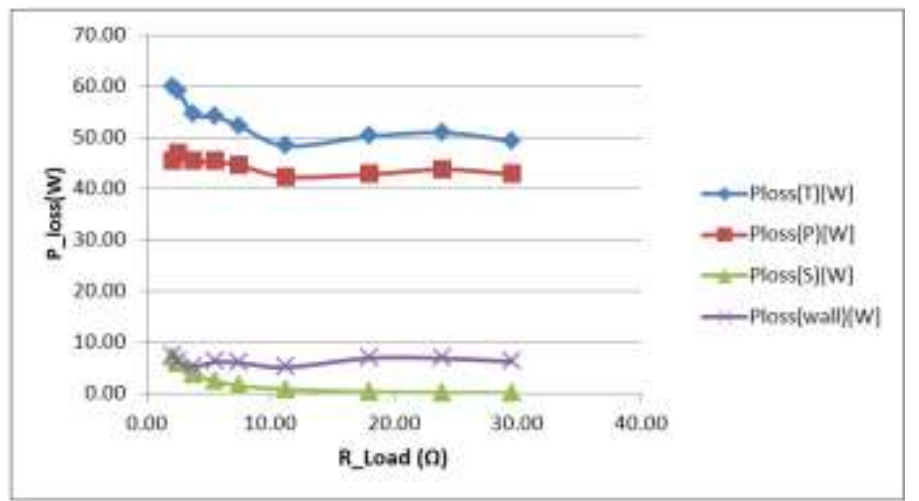

figure $4.8(b)$

Figure 4.8: (a) Plot of Input Power, output Power and efficiency against Load at 40Hz, (b) Plot of Losses against the Load at $40 \mathrm{~Hz}$

Table 4.5: varying Load $(R)$ at frequency of $(50 \mathrm{~Hz})$

\begin{tabular}{|c|c|c|c|c|c|c|c|c|c|c|c|c|c|}
\hline RL(Meaured)[(] & U1[V] & I1[A] & pf1 & P1[W] & U2[V] & I2[A] & pf2 & \multicolumn{2}{|c|}{ P2[W] } & eff (50Hz) Ploss(T)[W] Ploss(P)[W]Ploss(S)[W]Ploss(wall)[W] \\
\hline 2.08 & 30.50 & 3.12 & 0.58 & 55.19 & 4.02 & 1.930 & 1.00 & 7.76 & 14.06 & 47.43 & 34.27 & 7.19 & 5.98 \\
\hline 2.55 & 30.80 & 3.16 & 0.56 & 54.50 & 4.47 & 1.750 & 1.00 & 7.82 & 14.35 & 46.68 & 35.15 & 5.91 & 5.62 \\
\hline 3.80 & 30.50 & 3.09 & 0.54 & 50.89 & 5.43 & 1.430 & 1.00 & 7.76 & 15.26 & 43.13 & 33.61 & 3.95 & 5.57 \\
\hline 5.55 & 30.50 & 3.02 & 0.52 & 47.90 & 6.39 & 1.150 & 1.00 & 7.35 & 15.34 & 40.55 & 32.10 & 2.55 & 6.77 \\
\hline 7.52 & 30.40 & 2.98 & 0.49 & 44.39 & 7.01 & 0.932 & 1.00 & 6.53 & 14.72 & 37.86 & 31.26 & 1.68 & 5.69 \\
\hline 11.30 & 30.70 & 2.97 & 0.48 & 43.77 & 7.77 & 0.687 & 1.00 & 5.34 & 12.20 & 38.43 & 31.05 & 0.91 & 6.47 \\
\hline 18.00 & 30.50 & 2.94 & 0.46 & 41.25 & 8.39 & 0.466 & 1.00 & 3.91 & 9.48 & 37.34 & 30.43 & 0.42 & 6.49 \\
\hline 23.96 & 30.50 & 2.94 & 0.45 & 40.35 & 8.65 & 0.361 & 1.00 & 3.12 & 7.74 & 37.23 & 30.43 & 0.25 & 6.55 \\
\hline 29.57 & 30.70 & 2.95 & 0.44 & 39.85 & 8.87 & 0.300 & 1.00 & 2.66 & 6.68 & 37.19 & 30.63 & 0.17 & 6.38 \\
\hline
\end{tabular}

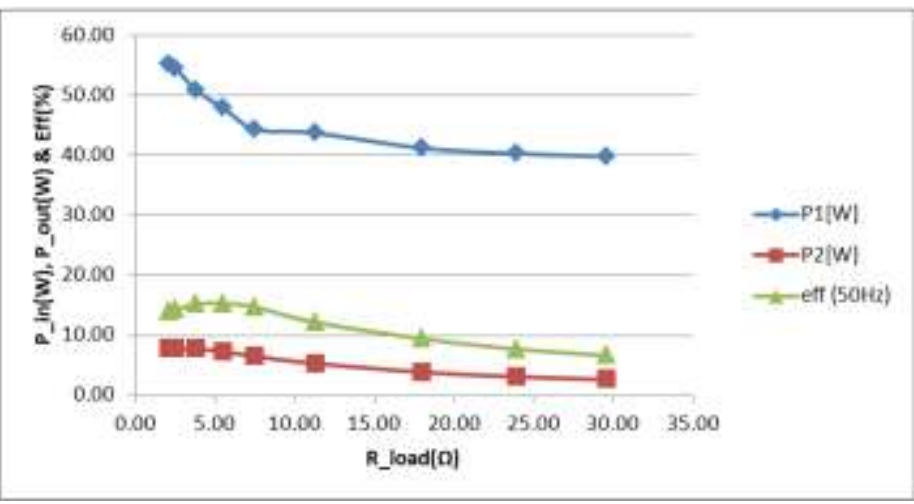

figure 4.9 (a)

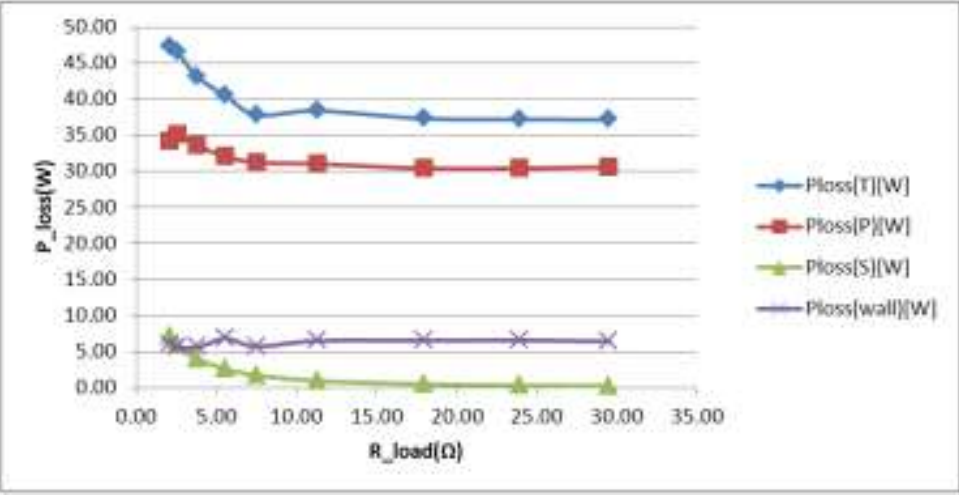

figure 4.9 (b)

Figure 4.9: (a) Plot of Input Power, output Power and efficiency against Load at 50Hz.,(b) Plot of Losses against the Load at $50 \mathrm{~Hz}$ 
Table 4.6: varying Load $(R)$ at frequency of $(70 \mathrm{~Hz})$

\begin{tabular}{|c|c|c|c|c|c|c|c|c|c|c|c|c|c|}
\hline R(Meaured) & U1(V) & I1(A) & pf1 & P1 & U2 & I2 & pf2 & P2 & eff(70Hz) Ploss(T) & Ploss(P) & Ploss(S) & Ploss(wall) \\
\hline 2.08 & 30.50 & 2.52 & 0.55 & 42.27 & 3.87 & 1.860 & 1.00 & 7.20 & 17.03 & 35.07 & 22.35 & 6.68 & 6.04 \\
\hline 2.55 & 30.60 & 2.54 & 0.54 & 41.97 & 4.33 & 1.700 & 1.00 & 7.36 & 17.54 & 34.61 & 22.71 & 5.58 & 6.32 \\
\hline 3.80 & 30.60 & 2.45 & 0.52 & 38.98 & 5.35 & 1.410 & 1.00 & 7.54 & 19.35 & 31.44 & 21.13 & 3.84 & 6.48 \\
\hline 5.55 & 30.30 & 2.34 & 0.50 & 35.45 & 6.32 & 1.140 & 1.00 & 7.20 & 20.32 & 28.25 & 19.27 & 2.51 & 6.46 \\
\hline 7.52 & 30.50 & 2.31 & 0.48 & 33.82 & 7.10 & 0.944 & 1.00 & 6.70 & 19.82 & 27.12 & 18.78 & 1.72 & 6.61 \\
\hline 11.30 & 30.50 & 2.24 & 0.46 & 31.43 & 7.82 & 0.692 & 1.00 & 5.41 & 17.22 & 26.02 & 17.66 & 0.92 & 7.43 \\
\hline 18.00 & 30.30 & 2.21 & 0.43 & 28.79 & 8.51 & 0.473 & 1.00 & 4.03 & 13.98 & 24.77 & 17.19 & 0.43 & 7.15 \\
\hline 23.96 & 30.40 & 2.20 & 0.42 & 28.09 & 8.78 & 0.366 & 1.00 & 3.21 & 11.44 & 24.88 & 17.04 & 0.26 & 7.58 \\
\hline 29.57 & 30.50 & 2.19 & 0.41 & 27.39 & 9.05 & 0.306 & 1.00 & 2.77 & 10.11 & 24.62 & 16.88 & 0.18 & 7.55 \\
\hline
\end{tabular}

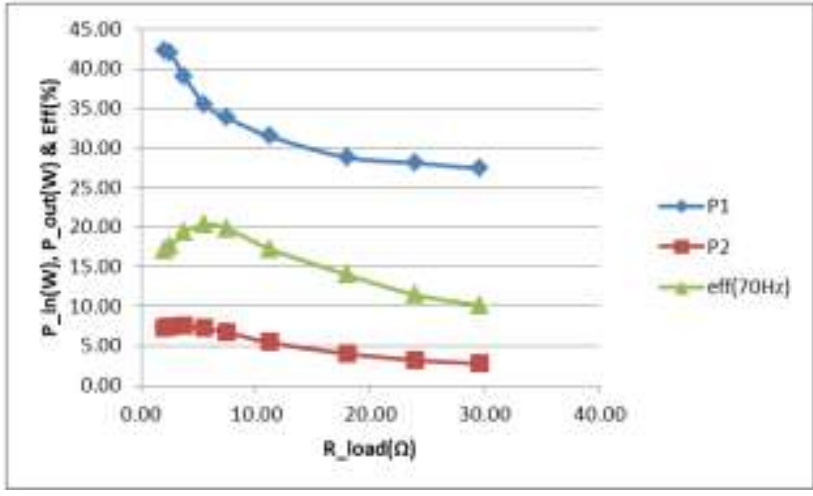

figure $4.10(a)$

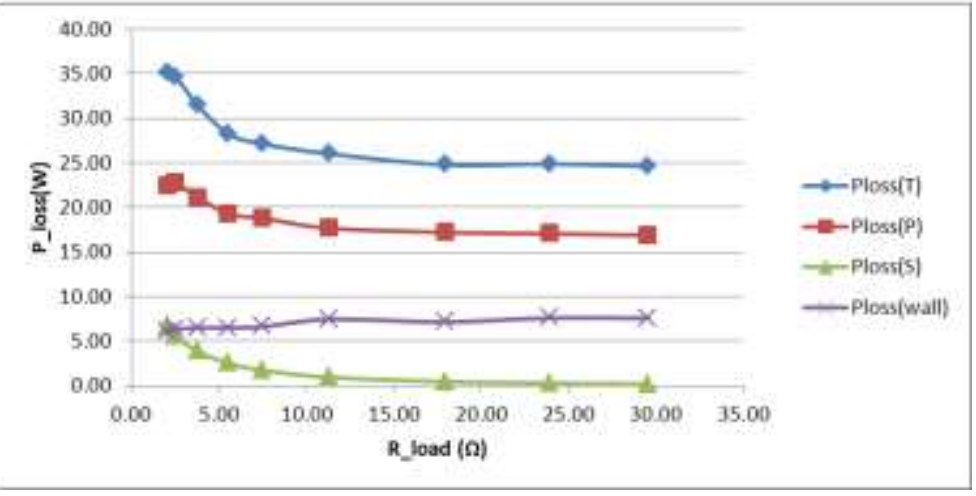

figure $4.10(b)$

Figure 4.10: (a) Plot of Input Power, output Power and efficiency against Load at 70Hz.,(b) Plot of Losses against the Load at $70 \mathrm{~Hz}$

Table 4.7: varying Load $(R)$ at frequency of $(100 \mathrm{~Hz})$

\begin{tabular}{|c|c|c|c|c|c|c|c|c|c|c|c|c|c|}
\hline$R$ (Meaured) & U1(V) & I1(A) & pf1 & P1 & U2 & I2 & pf2 & P2 & eff $(100 \mathrm{~Hz})$ & Ploss(T) & Ploss(P) & Ploss(S) & Ploss(wall) \\
\hline 2.08 & 30.40 & 2.00 & 0.54 & 32.83 & 3.50 & 1.680 & 1.00 & 5.88 & 17.91 & 26.95 & 14.08 & 5.45 & 7.42 \\
\hline 2.55 & 30.50 & 2.01 & 0.53 & 32.49 & 3.90 & 1.530 & 1.00 & 5.97 & 18.36 & 26.52 & 14.22 & 4.52 & 7.79 \\
\hline 3.80 & 30.50 & 1.92 & 0.53 & 31.04 & 4.98 & 1.310 & 1.00 & 6.52 & 21.02 & 24.51 & 12.98 & 3.31 & 8.22 \\
\hline 5.55 & 30.40 & 1.83 & 0.52 & 28.93 & 6.10 & 1.100 & 1.00 & 6.71 & 23.20 & 22.22 & 11.79 & 2.34 & 8.10 \\
\hline 7.52 & 30.40 & 1.77 & 0.51 & 27.44 & 6.83 & 0.910 & 1.00 & 6.22 & 22.65 & 21.23 & 11.03 & 1.60 & 8.60 \\
\hline 11.30 & 30.30 & 1.69 & 0.48 & 24.58 & 7.75 & 0.686 & 1.00 & 5.32 & 21.63 & 19.26 & 10.05 & 0.91 & 8.30 \\
\hline 18.00 & 30.30 & 1.64 & 0.46 & 22.86 & 8.47 & 0.471 & 1.00 & 3.99 & 17.45 & 18.87 & 9.47 & 0.43 & 8.97 \\
\hline 23.96 & 30.30 & 1.62 & 0.44 & 21.60 & 8.73 & 0.365 & 1.00 & 3.18 & 14.73 & 18.42 & 9.24 & 0.26 & 8.92 \\
\hline 29.57 & 30.30 & 1.61 & 0.43 & 20.98 & 8.89 & 0.301 & 1.00 & 2.67 & 12.74 & 18.31 & 9.12 & 0.17 & 9.01 \\
\hline
\end{tabular}




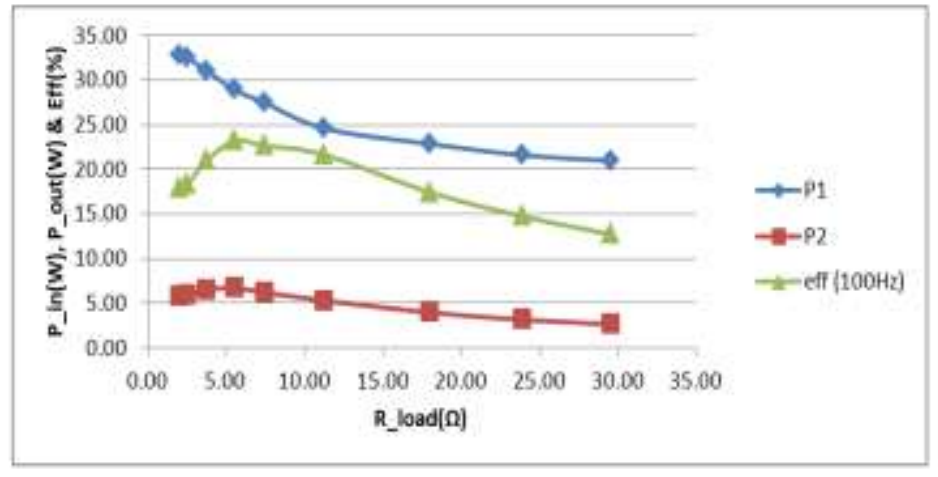

figure 4.11 (a)

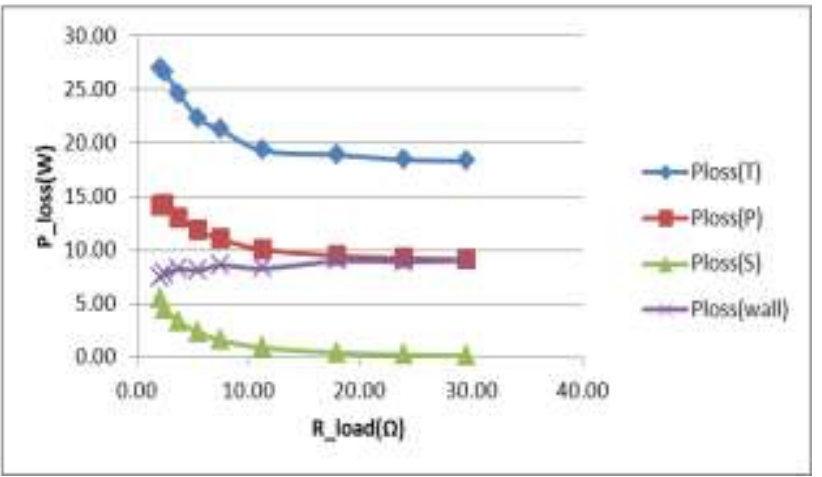

figure 4.11 (b)

Figure 4.11: (a) Plot of Input Power, output Power and efficiency against Load at 100Hz.,(b) Plot of Losses against the Load at $100 \mathrm{~Hz}$

It is also important to see what happens if the frequency is varied to see the amount of power transfer at an optimum load. However, since from the four frequencies measurements above $R=5.55 \Omega$ seems to be an average optimum load. This optimum load is determined experimentally by varying the load between $0 \Omega$ and $30 \Omega$ for those chosen four frequency. Table 4.8 shows the measurements made by varying the frequency and the plot made from the table are shown in figure 4.12

Table 4.8: varying frequency at constant Load $(R)$

\begin{tabular}{|c|c|c|c|c|c|c|c|c|c|c|c|c|c|}
\hline freq [Hz] & U1 [V] & I1 [A] & pf1 & P1 [W] & U2 [V] & I2 [A] & pf2 & $\mathbf{P} 2[\mathrm{~W}]$ & eff $(5.55 \Omega)$ & Ploss(T) [W] & Ploss(P) [W] & Ploss(S)[W] & Ploss(wall)[W] \\
\hline 50 & 30.5 & 3.02 & 0.52 & 47.90 & 6.39 & 1.15 & 1.00 & 7.35 & 15.34 & 40.55 & 32.10 & 2.55 & 5.89 \\
\hline 100 & 30.4 & 1.83 & 0.52 & 28.93 & 6.1 & 1.1 & 1.00 & 6.71 & 23.20 & 22.22 & 11.79 & 2.34 & 8.10 \\
\hline 200 & 30.3 & 1.16 & 0.51 & 17.93 & 4.8 & 0.863 & 1.00 & 4.14 & 23.11 & 13.78 & 4.74 & 1.44 & 7.61 \\
\hline 300 & 30.2 & 0.88 & 0.49 & 12.95 & 3.77 & 0.673 & 1.00 & 2.54 & 19.60 & 10.41 & 2.70 & 0.87 & 6.84 \\
\hline 500 & 30.0 & 0.58 & 0.46 & 8.05 & 2.5 & 0.452 & 1.00 & 1.13 & 14.05 & 6.92 & 1.20 & 0.39 & 5.32 \\
\hline
\end{tabular}

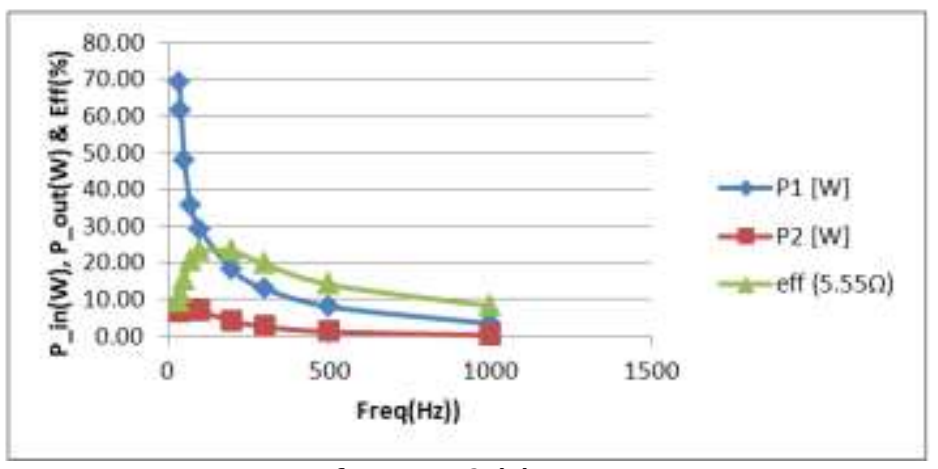

figure 4.12 (a)

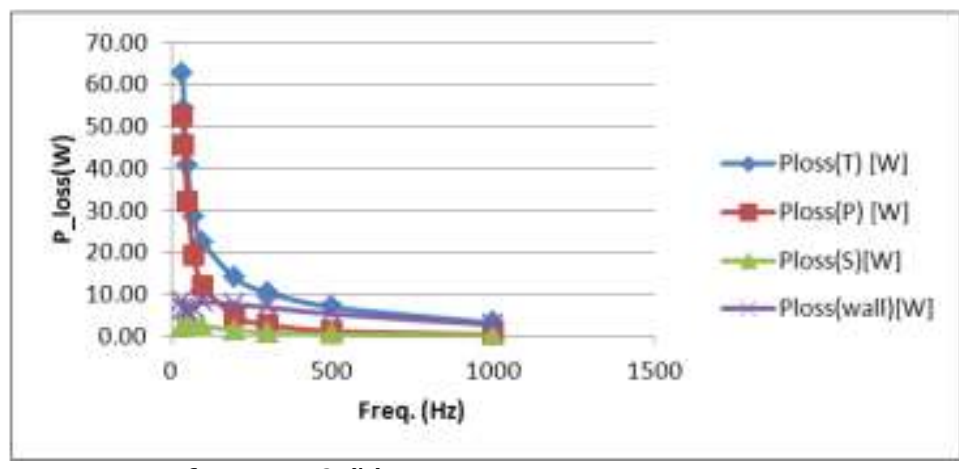

figure 4.12 (b)

Figure 4.12: (a) Plot of Input Power, output Power and efficiency against frequency at $5.55 \Omega$ (b) Plot of Losses against frequency at $5.55 \Omega$ 
In order to determine the magnetic flux at the surface of the tube as a function of frequency as we need to measure no-load voltages for each frequency used. If we apply faraday's law to primary coil in figure 4.5

$$
\oint_{C} \vec{E} \cdot \vec{\tau} d l=-\frac{d}{d t} \iint_{S} \vec{B} \cdot \vec{n} d A
$$

Where $\vec{E}$ is electric field, $d l$ small element of the coil, $\vec{B}$ is magnetic flux density, $d A$ small area elemental whose magnitude is the area of the infinitesimal surface and $\vec{\tau}$ and $\vec{n}$ are unit vector respectively.

The right part of the equation is equal to no-load voltage and if we use phasor representation with the assumption that the flux is uniform, for one turn the equation becomes

$$
U_{N L}=\omega B_{i n} A_{2}
$$

Therefore, for $\mathrm{N}$ number of turns, we can write

$$
U_{N L}=N_{2} \omega B_{i n} A_{2}
$$

Thus, it is necessary to do no load measurements at each frequency used above. Table 4.9 shows this measurement comparison. Since we know the secondary coil number of turns and coil area and $B_{i n}$, we now determine the no-load induced voltage. The plot made from this table is compared with the one made from analytical model in Appendix A.2 (figure A.1)

Table 4.9: No-load measurements at different frequency

\begin{tabular}{|c|c|c|c|c|c|c|}
\hline freq [Hz] & Unl1[V] & Inl1 [A] & Bin (Expt)[T] & Bin (Anayl)[T] & Unl2(Expt)[V] & Unl2(Anayl)[V] \\
\hline 40 & 31.1 & 3.630 & 0.0090 & 0.0100 & 9.48 & 10.51 \\
\hline 50 & 30.8 & 2.970 & 0.0074 & 0.0081 & 9.68 & 10.64 \\
\hline 70 & 30.6 & 2.200 & 0.0054 & 0.0059 & 9.87 & 10.85 \\
\hline 100 & 30.5 & 1.600 & 0.0038 & 0.0042 & 9.95 & 11.04 \\
\hline 200 & 30.5 & 0.895 & 0.0019 & 0.0022 & 9.81 & 11.56 \\
\hline 300 & 30.3 & 0.669 & 0.0012 & 0.0015 & 9.49 & 11.83 \\
\hline 500 & 30.2 & 0.478 & 0.0007 & 0.0009 & 8.80 & 11.83 \\
\hline 1000 & 29.9 & 0.301 & 0.0003 & 0.0004 & 7.00 & 10.51 \\
\hline
\end{tabular}




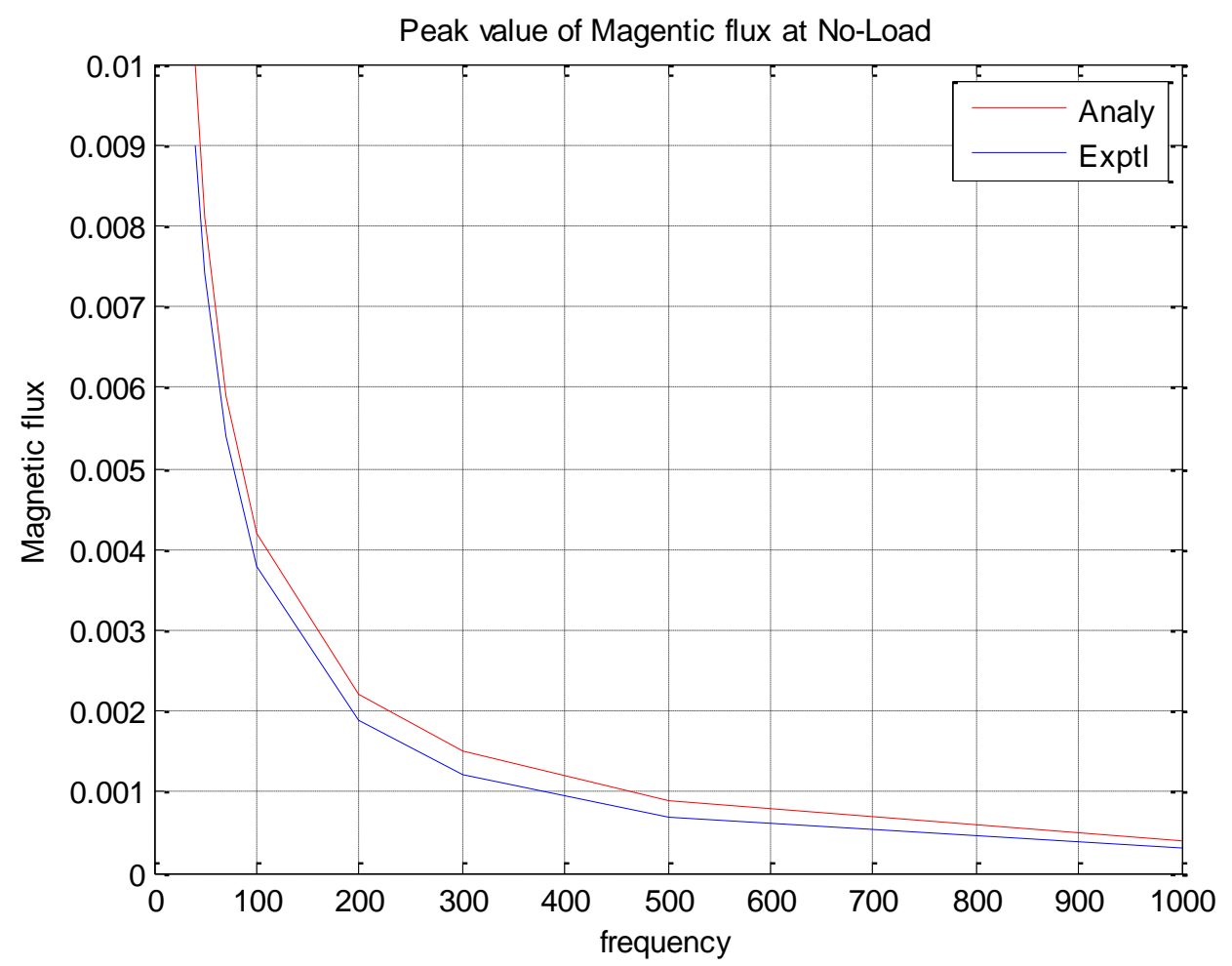

Figure 4.13: Magnetic flux comparison of experimental and analytical against frequency at no-load

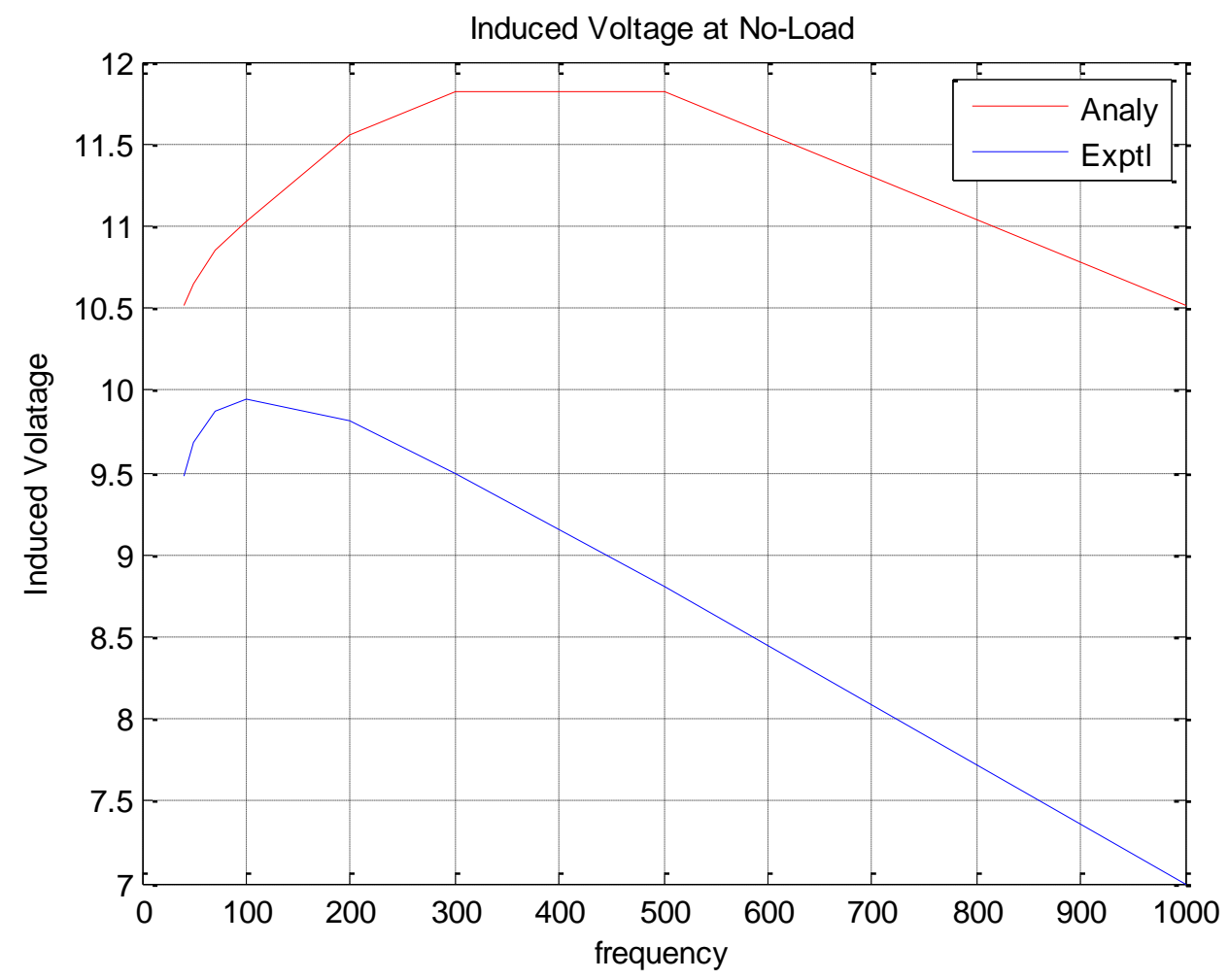

Figure 4.14: Induced Voltage comparison of experimental and analytical against frequency at no-load 


\subsubsection{Ferro-steel measurement Results}

All measurements tried on the ferro-steel do not give desirable results. It was difficult to see any power delivered to the load even when the maximum input power was applied. However, at very low frequency between $(5 \mathrm{~Hz}-10 \mathrm{~Hz})$, there is No load voltage of $73 \mathrm{mV}$ and open circuit current of $23 \mathrm{~mA}$.

\subsection{Discussion of Results}

We noticed that power is transferred from primary to secondary coil via the tube in figure 4.5 in our experiment, but this has to be validated by the analytical model made in chapter three and arrive at logical comparisons.

We shall use table 4.9 for our first comparison. $B_{\text {in }}$ (Expt) is the experimental calculated value of magnetic flux at the surface of the pipe in table 4.9 while $B_{\text {in }}$ (Anayl) is the analytical value of generated with from matlab script in appendix A.2. The experiment shows the real life behaviour of the magnetic field at the surface of the pipe and is different from the analytical because of assumptions in section 3.1 to arrive at a logical analysis besides generating the measurements at no-load condition. One assumption is the ferromagnetic case used in analytical in not used in the experiment, also the coils seem to be very close in analytical while there is a container holding the coil in practice leas to more gaps between the coil and the tube. However, the behaviour of the flux density as a function of frequency remains the same. Figure 4.14 shows the comparison in graphical form.

In our second comparison, we shall look into the No-load losses in the wall as the frequency increases. Table 4.10 shows the comparison of losses calculation from the analytical model in appendix A.2 and the measured values for the experiments. These results are presented in graphical form in figure 4.15 .

Table 4.10: Wall losses comparison at No- load

\begin{tabular}{|c|c|c|}
\hline freq [Hz] & Ploss(wall)-ExptI[W] & Ploss(wall)-Analyl[W] \\
\hline 40 & 7.8060 & 15.0236 \\
\hline 50 & 7.3704 & 14.3687 \\
\hline 70 & 7.8716 & 13.5373 \\
\hline 100 & 10.0208 & 12.5603 \\
\hline 200 & 9.7372 & 11.3946 \\
\hline 300 & 9.1681 & 9.5317 \\
\hline 500 & 7.5684 & 7.0664 \\
\hline 1000 & 4.3610 & 3.8354 \\
\hline
\end{tabular}




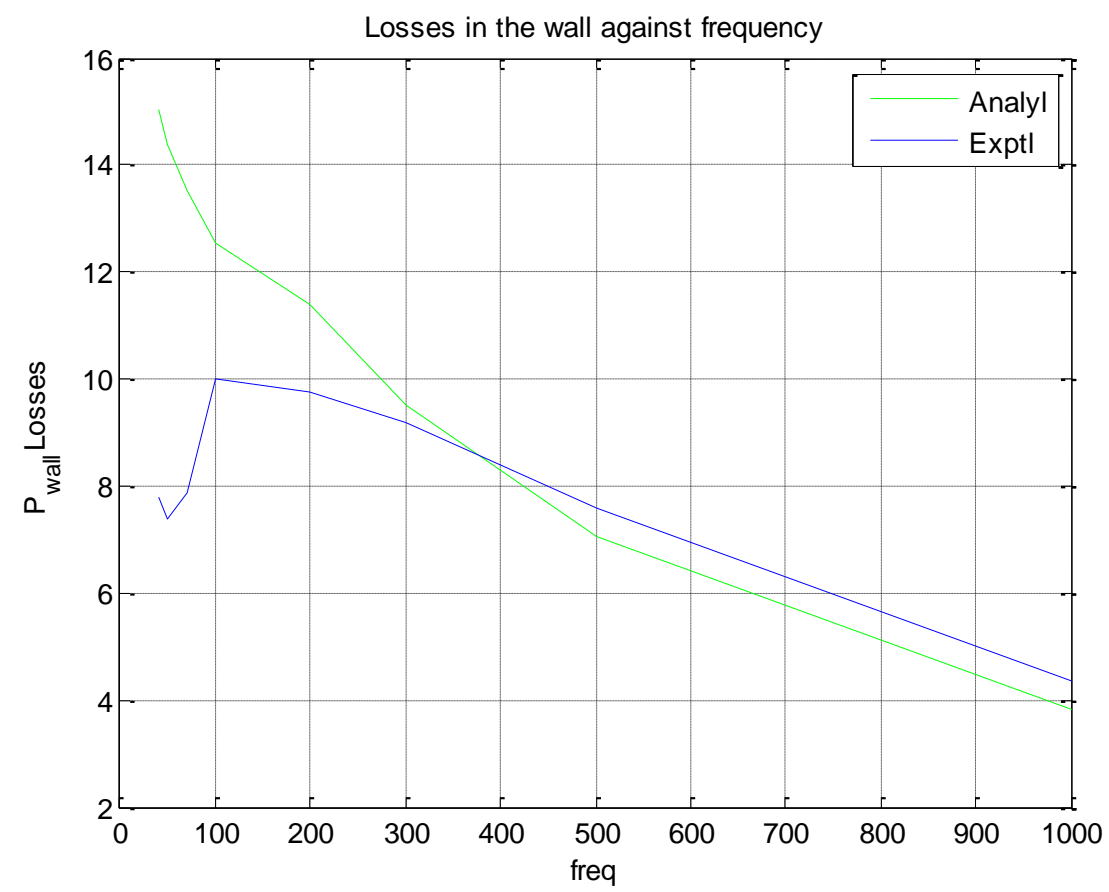

Figure 4.15: comparison of experimental and analytical plots of losses against frequency at no-load

At the lower frequency the losses in the wall are over estimated in analytical model in comparison with the experiment, while at the higher frequency the losses in the wall are under estimated. This is because of the area assumed at lower frequency and skin depth effect at high frequency.

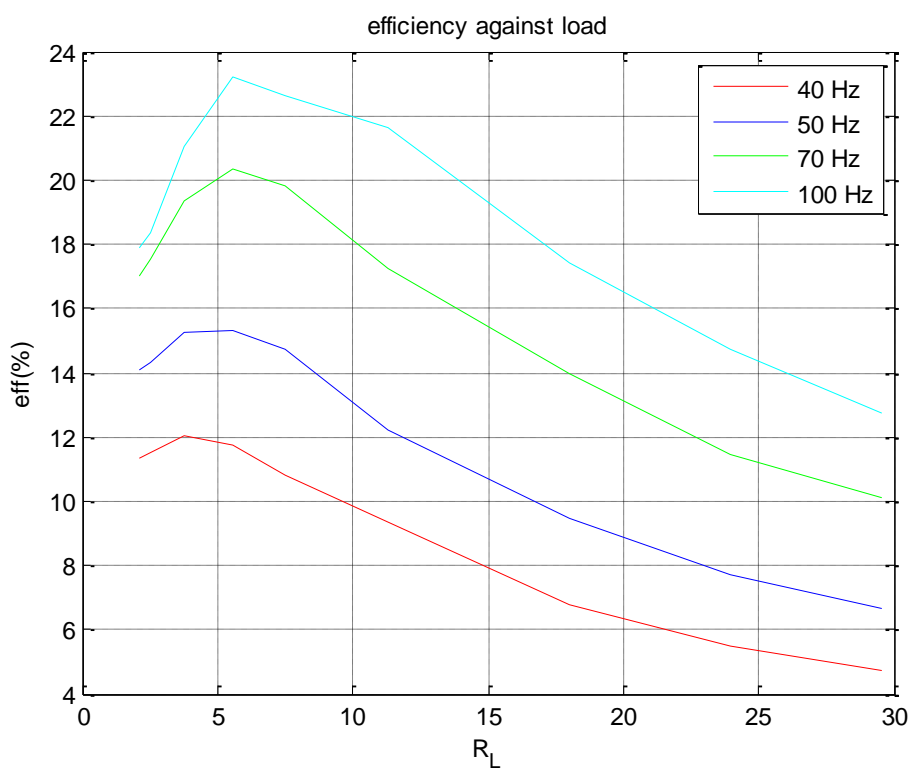

Figure 4.16: Efficiency against Load for each frequency

Figure 4.16 shows the comparison of the efficiencies of $(40 \mathrm{~Hz}, 50 \mathrm{~Hz}, 70 \mathrm{~Hz}$ and $100 \mathrm{~Hz})$ obtained from the tables $4.4-4.7$ by varying the load at each frequency. This mean there should be certain optimum 
frequency for power transfer via the pipe. The experiment was only done for four different frequencies, however efficiency is expected to decrease at high frequency due to skin effect of the pipe. 
Chapter 5

\section{Conclusion and Recommendations}

The thesis deals with inductive power transfer via a metal cylindrical pipe. As described in chapter one, the objective of the thesis is to explore the possibilities of transferring power from an energised primary coil via the pipe's wall to a secondary coil. In Chapter three we discussed the analytical model which was compared with the experimental model in chapter four. We shall discuss conclusion and recommendations for further research in this chapter

\subsection{Conclusions}

The eddy currents and losses due to eddy currents can be modeled analytically. However, a number of assumptions are required to simplify analytical modeling. This leads to some discrepancies in the measured and predicted values of losses. The losses in the analytical model are over estimated at lower frequency and under estimated at high frequency when compared with the experimental losses in the wall of the pipe.

Also the analytical values of magnetic flux density in the tube are found to be about 1.1 times more than the ones determined experimentally. This is because in analytical model, an ideal iron core is used and the coil is very close to the surface of the pipe whereas in experimental model there is no ideal iron and there is gap between the coil and the pipe. With these two reasons and other assumptions made in analytical model we have such discrepancies.

Richard M. Nixon said and I quote "In theory, there is no difference between theory and practice. But, in practice, there is". Therefore the analytical (theory) gives quick view of what we expected in the wall of the pipe by simplifying the models using some assumption and the results has some degree of discrepancies with the experimental (practice) which gives the clear picture power is transferred from primary to the secondary. However they have a similar trend.

\subsection{Recommendations}

In this thesis, finding a model for inductive power transfer analytically has not been easy, but the experimental model has been interesting. However, we have identified the following for further research;

1. The experimental measurements made from the ferro-steel pipe are not what we desire because of the permeability; therefore if the ferro-steel is heated it may be possible to transfer some power from source to the load at a very low frequency. It will also be interesting to look into any other possibility of power transfer via this material medium

2. Simulation of the model is very vital; this will give a clearer picture of the experimental model. Therefore, we suggest a Finite Element Method (FEM) model as a possible 
continuation of this thesis. This will even bring more ideas that need further experimental proof.

3. A circuit model has been developed in chapter 3, the determination of the model is very important. This will be an interesting area for further investigation about IPT via a metal object. 


\section{References}

[1] Vandevoorde, G. and Puers, R. "Wireless Energy Transfer for Stand-Alone Systems: A Comparison Between Low and High Power Applicability." Sensors and Actuators (2001): 305 311.

[2] J. de Boeij, E Lomonova, J. L. Duarte, A.J.A. Vandernput., "Contactless power supply for moving sensors and actuators in high-precision mechatronic systems with long-stroke power transfer capability in x-y plane". Sensors and Actuators (2008): 319 - 328.

[3] http://en.wikipedia.org/wiki/Heinrich Hertz Accessed 10 April, 2010

[4] http://en.wikipedia.org/wiki/Nikola Tesla Accessed 10 April, 2010

[5] D. J. Cichon and W. Wiesbeck, "The Heinrich Hertz Wireless Experiment at Karlsruhe in view of Modern Communication.

[6] Brown, W. "The History of Power Transmission by Radio Waves." IEEE Transactions on

Microwave Theory and Techniques"Vol. MTT-32, No. 9 (September 1984): 1230 -1242.

[7] http://www.netsense.net/tesla/article3.html Accessed 10 April, 2010

[8] Vuckovic, J. I. "Nikola Tesla: The Man Time Forgot." IEEE Potentials (Oct 1990): 53- 54.

[9] Thomas K. Simpson,"Maxwell and the Direct Experimental Test of His Electromagnetic Theory", "The University of Chicago Press : The History of Science Society" Vol. 57, No. 4 (Winter, 1966), pp. 411-432

[10] http://users.tkk.fi/jwagner/tesla/tc-plans.htm Accessed 24 May, 2010

[11] D.A.G. Pedder, A.D. Brown. "A contactless electrical energy transmission system." IEEE Transactions on Industrial Electronics, Vol. 46, No. 1, February 1999, pp 23-30.

[12] Yungtaek Jang, Milan M. Jovanovic, A Contactless Electrical Energy Transmission System for Portable-Telephone Battery Chargers," IEEE Transactions on Industrial Electronics, VOL. 50, NO. 3, JUNE 2003: pp520-527

[13] A.J. MORADEWICZ1 and M.P. KAZMIERKOWSKI, High efficiency contactless energy transfer system with power electronic resonant converter, Bulletin of the Polish Academy of Sciences Technical Sciences, Vol. 57, No. 4, 2009 
[14] F. F. A. Van der Pijl, J. A. Ferreira, P. Bauer, H. Polinder, "Design of an Inductive Contactless Power System for Multiple Users", Conference Record of the 2006 IEEE 41st IAS Annual Meeting, Vol. 4, pp. 1876-1883, October 2006

[15] Chwei-Sen Wang, Oskar H. Stielau, and Grant A. Covic, Design Considerations for a Contactless Electric Vehicle Battery Charger, IEEE Transactions on Industrial Electronics, Vol. 52, no. 5, October 2005: pp1308-1314

[16] R. Laouamer, M. Brunello, J.P. Ferrieux, 0. Normand, N. Buchheit."A multi-resonant converter for non-contact charging with electromagnetic coupling." 23rd Intemational Conference on Industrial Electronics, Control and Instrumentation, IECON97, Vol 2, 1997, pp792-797

[17] H. Sakamoto, K. Harada, S. Washimiya, K. Takehara, Y. Matsuo, F. Nakao. "Large air-gap coupler for inductive charger [for electric vehicles]" IEEE Transactions on Magnetics, Vol. 35, no. 5, Sept. 1999: pp 3526 -3528.

[18] J. Lukacs, M. Kiss, I. Nagy, G. Gonter, R. Hadik, K. Kaszap. A. Tarsoly. "Inductive energy collection for electric vehicles." Conference Proceedings, Fourth Power Electronics Conference, Budapest, 1981: pp 71-81

[19] K. Lashkari, S. E. Schladover, E.H. Lechner. "Inductive power transfer to an electric vehicle." Proceedings of the 8"' International Vehicle Symposium, 1986, pp 258-267.

[20] Juan Luis Villa , Jesús Sallán, Andrés Llombart, José Fco Sanz, "Design of a high frequency Inductively Coupled Power Transfer system for electric vehicle battery charge", Applied Energy, Volume 86, Issue 3, March 2009, Pages 355-363

[21] A. Esser. H.-C. Skudelny. "A new approach to power supplies for robots." IEEE Transactions on Industry Applications, Vol. 27, no. 5. Sept.-Oct. 1991, pp 872-875

[22] A. Kawamun, K. Ishioka, J. Hirai. "Wireless transmission of power and information through one high frequency resonant $\mathrm{AC}$ link inverter for robot manipulator applications" Conference Record of the $13^{\text {th }}$ IEEE Industry Applications Conference Meeting, IAS '95. Vol. 3, 1995, pp 23672372.

[23] J.M. Bamard, J.A. Ferreira, J.D. van Wyk. "Sliding transformers for linear contactless power delivery" IEEE Transactions on Industrial Electronics, Vol. 44. no. 6, Dec. 1997, pp 774-779.

[24] B.J. Heeres, D.W. Novotny, D.M. Divan, R.D. Lorenz. Contactless underwater power delivery PESC Record - IEEE Annual Power Electronics Specialists Conference, Vol. 1, Jun 20-24 1994, pp 418-423,0275-9306. 
[25] Potterill R D H " The Development of Electromagnetic Theory and its Application to Telegraphy" April 26, 2007

[26] http://en.wikipedia.org/wiki/Electromagnetic induction Accessed 10 April, 2010

[27] http://en.wikipedia.org/wiki/Nicholas Callan, Accessed 10 April, 2010

[28] Thomas B. Greenslade, Jr. "Instruments for Natural Philosophy" http://physics.kenyon.edu/EarlyApparatus/Electricity/Electrotome or Repeater/Electrotome.html

[29] Yue Tsan Lee, Electric Stove with electromagnetic induction cooker and hot plate, U. S. Patent. 5280152, Jan. 1994.

[30] R. D. Dowling et al., Initial experience with the AbioCor implantable replacement heart system, Journal of Thoracic and Cardiovascular Surgery, Volume 127, Issue 1, January 2004, Pages 131-141

[31] Hans-Jurgen Brockmann et al, Charger with inductive power transmission for batteries in a mobile, U. S. Patent. 6118249, Sept. 2000.

[32] http://www.powercastco.com Accessed 10 April, 2010

[33] Leopold Summerer, Oisin Purcell, "Concepts for wireless energy transmission via laser" (ESA - Advanced Concepts Team Keplerlaan 1, NL-2201AZ Noordwijk, The Netherlands)

[34]G. A. Landis. Moonbase night power by laser illumination. Journal of Propulsion and Power, 8, 1992

[35] G. A. Landis. Applications for space power by laser transmission. In Proceedings SPIE, volume 2121, pages 252-255, 1994.

[36] M. Bauer, P. Becker and Q. Zheng, Inductive power supply (IPSß) for the transrapid, ThyssenKrupp Transpid GmbH, Munich, Germany.

[37] http://www.electrosmart.net/electricity/inductive power transfer.php Accessed 10 April, 2010

[38] http://www.hardingtraffic.co.nz/ Accessed 10 April, 2010

[39] M. Justin, Smart studs keep an eye on road conditions, New Scientist. Vol. 146, no. 1972, Apr. 1995: pp. 20-20.

[40] Richard L. Stoll, The Analysis of Eddy Currents, Oxford University press 1974 
[41] Sheppard J. Salon, Finite Element Analysis of Electrical Machines, Kluwer Academic publisher, 1995

[42] Bay F, Labbe V, Favennec $Y$, Chenot JL. A numerical model for induction heating processes coupling electromagnetism and thermomechanics. International Journal for Numerical Methods in Engineering 2003; 58: 839-867

[43] B. Funieru and A. Binder, "Losses and forces due to eddy currents in a magnetically nonlinear conductive half space," Electr. Eng. (Archiv für Elektrotechnik), vol. 90, no. 1, pp. 43-54, Nov. 2007

[44] http://en.wikipedia.org/wiki/Heat equation

[45]Ferreira J. A. "Power Electromagnetics- ET4003", Faculteit EWI, 2007

[46] Ulrich R. Christenen, et al, 'Energy flux determines magnetic field strength of planet and stars", NATURE, Vol 47, Jan 2009

[47] Lehmann T. H., "The calculation of Magnetic Attraction" ,Transactions , AIEE, pp 383-394

[48] http://www.vanleeuwenstainless.nl/

[49] http://www.vanleeuwenbuizen.nl/

[50] F. W. Grover. , Inductance Calculations-Working formulas and tables, ISA, 1973

[51] (http://www.technick.net/public/code/cp dpage.php?aiocp dp=util_inductance circle) 


\section{Appendices}

\section{Appendix A}

\section{Matlab files}

In this appendix the matlab scripts created and used are given.

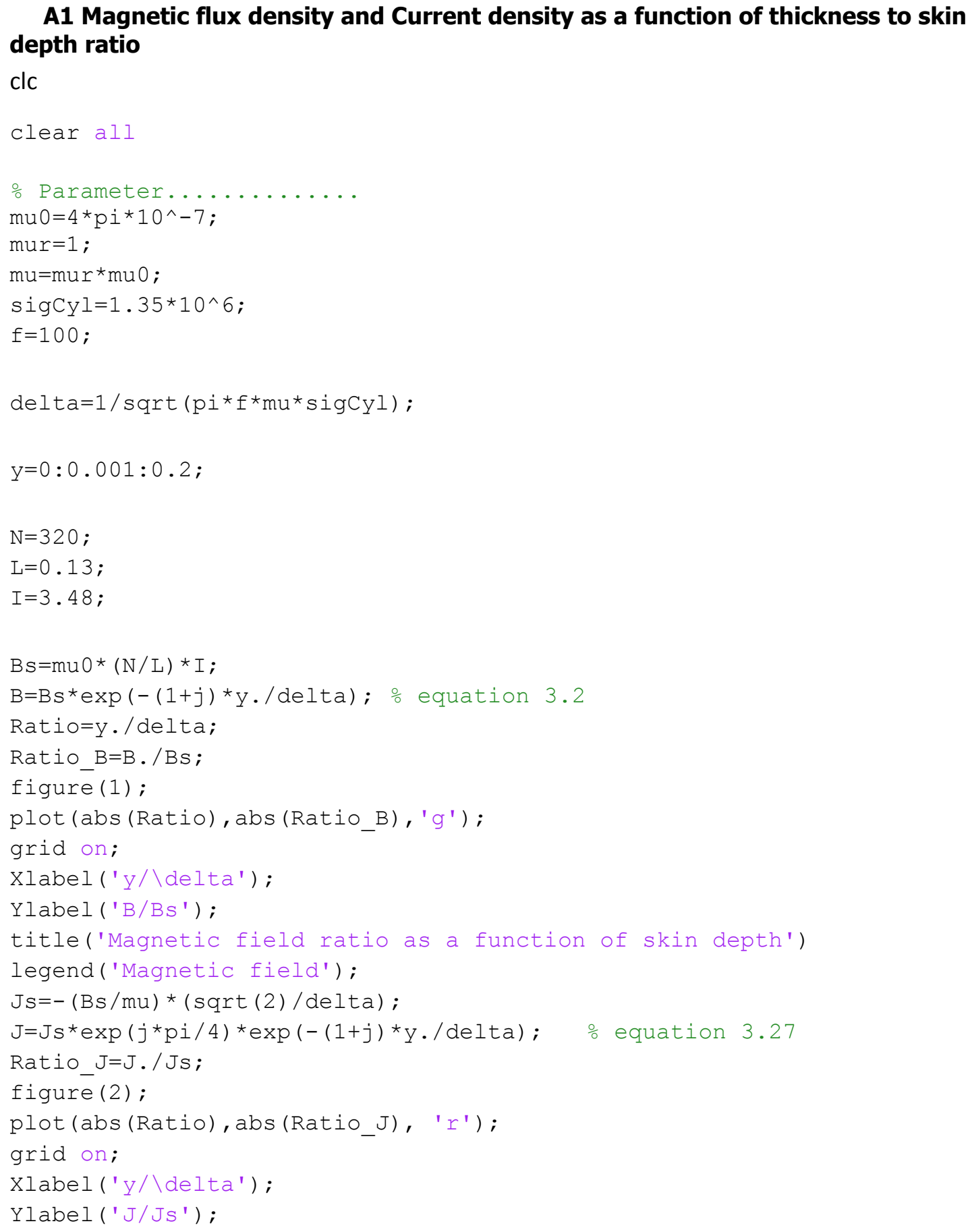


title('Current density ratio as a function of skin depth')

legend('Current density');

\section{A2: No-load (Induced and Magnetic flux) as a function of frequency}

$\mathrm{clc}$

clear all

ㅇ Parameter................

$f=\left[\begin{array}{llllllll}40 & 50 & 70 & 100 & 200 & 300 & 500 & 1000\end{array}\right]$; $\%$ frequency used in the experiment

omg $=2 * p i . * f ;$

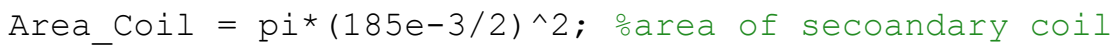

$\mathrm{N}=220$; onumber of turns of sec coil

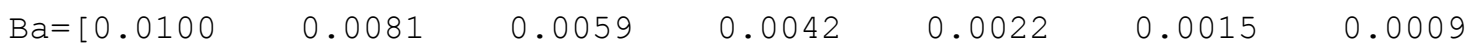

0.0004]; opeak value of Magentic flux (Analytical) at no_load for each each frequency

$\therefore \mathrm{Be}=\left[\begin{array}{lllllll}0.0064 & 0.0052 & 0.0038 & 0.0027 & 0.0013 & 0.0009 & 0.0005\end{array}\right.$

$0.0002]$;

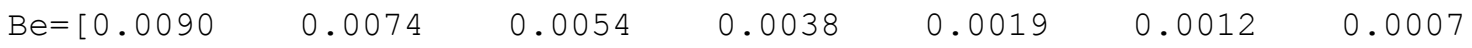

0.0003]; ․eak value of Magentic flux (Experimental) at no_load for each each frequency

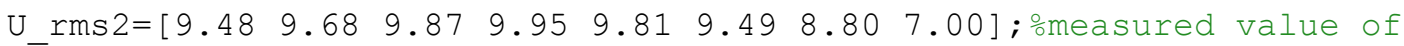

Iñduced Volatage @ No_load

const $=\mathrm{N} .{ }^{\star}$ omg *Area_Coil;

$\mathrm{U} 1=$ const. ${ }^{\star} \mathrm{Ba}$;

$\mathrm{U}_{-} \mathrm{rms} 1=\mathrm{U} 1 . / \operatorname{sqrt}(2)$;

figure (1);

$\operatorname{plot}\left(\mathrm{f}, \mathrm{Ba}, \mathrm{Cr}^{\prime}, \mathrm{f}, \mathrm{Be}, \mathrm{b}^{\prime}\right)$;

legend ('Analy', 'Exptl') ;

grid on;

Xlabel ('frequency') ;

Ylabel ('Magnetic flux');

title('Magentic flux at No-Load');

figure (2);

plot (f, U_rms1, 'r', f, U_rms2, 'b') ;

legend ('Ānaly', 'Exptl');

grid on;

Xlabel ('frequency' ) ;

Ylabel ('Induced Volatage') ;

title('Induced Voltage at No-Load'); 


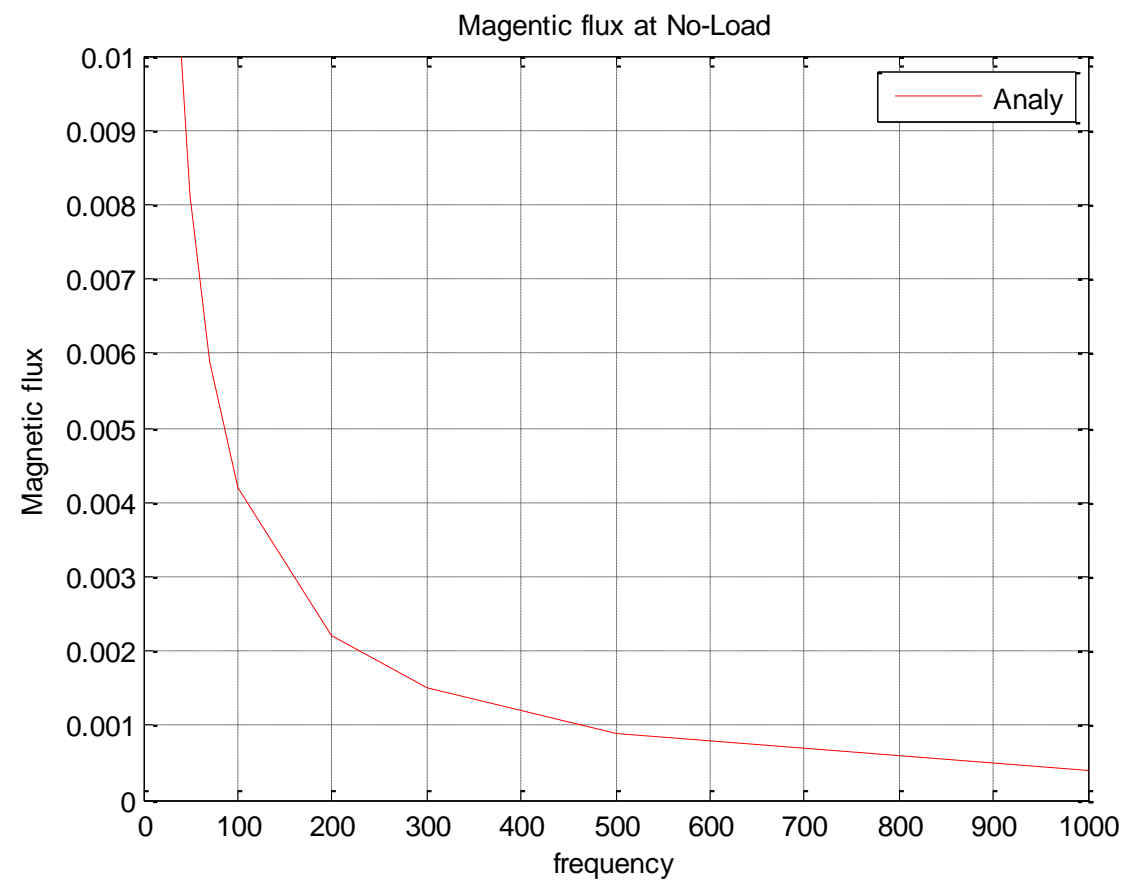

Figure A.1: Analytical value of Magnetic flux as a function of frequency at no-load

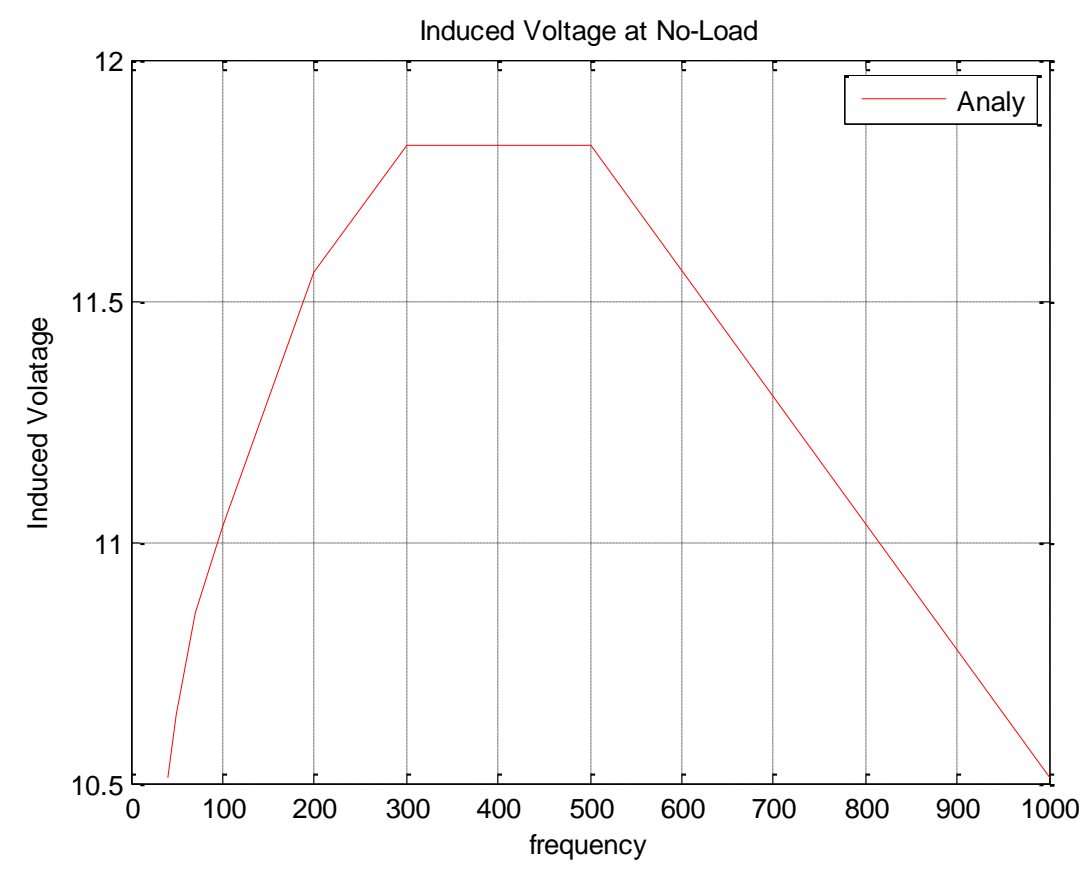

Figure A.2: Analytical value of Induced voltage as a function of frequency at No-Load

\section{A3: Eddy current Losses at $40 \mathrm{~Hz}$ when varying Load resistance}

$\mathrm{ClC}$

clear all 


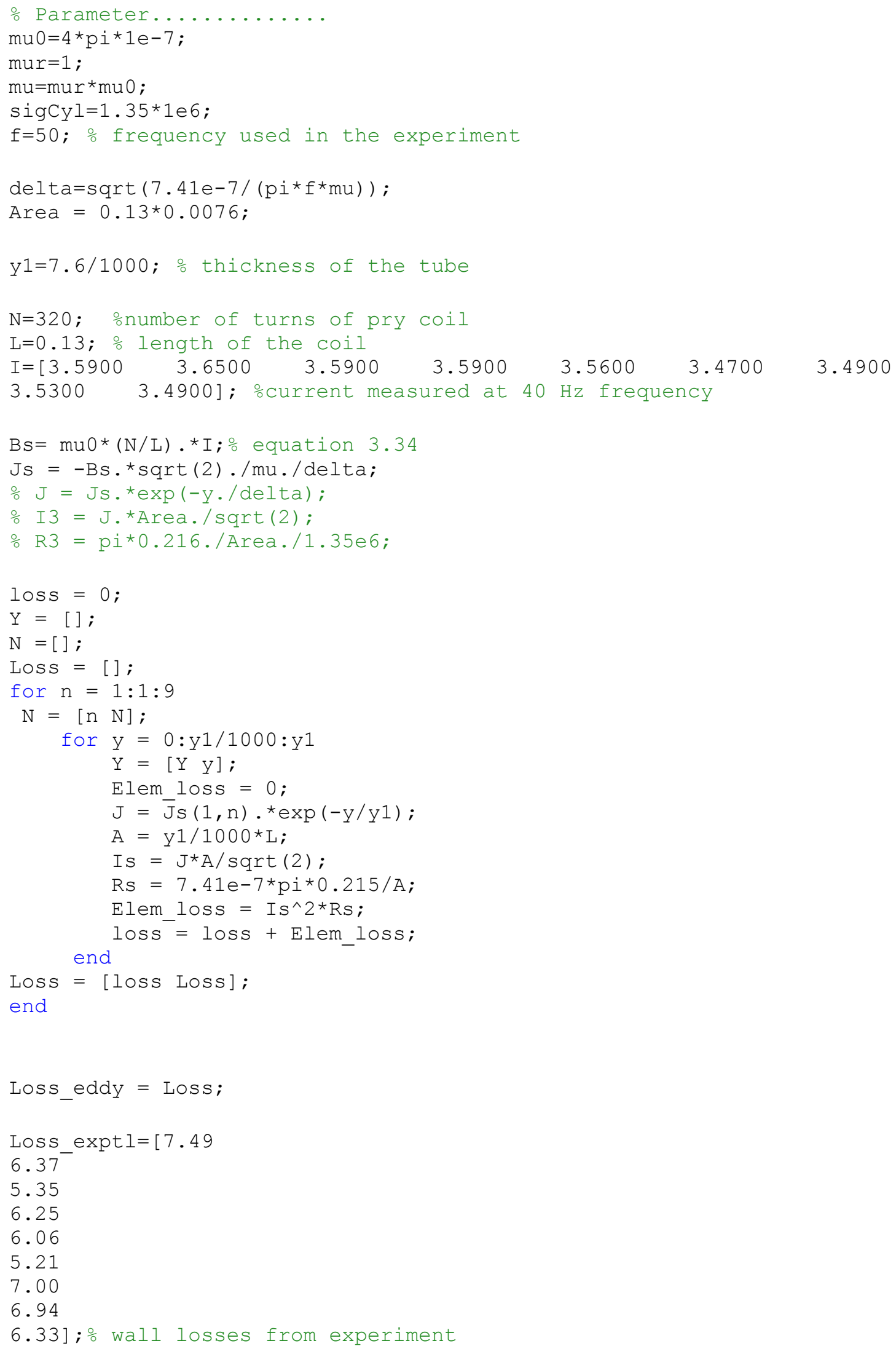


figure (3) ;

plot (R_L, Loss_eddy , 'r', R_L, Loss_exptl, 'b') ;

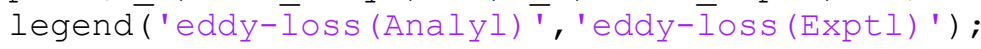

grid on;

Xlabel ('R_ $L\}$ ') ;

Ylabel ('P- wall $^{-}$');

title('Losses a (40Hz)in the wall against load');

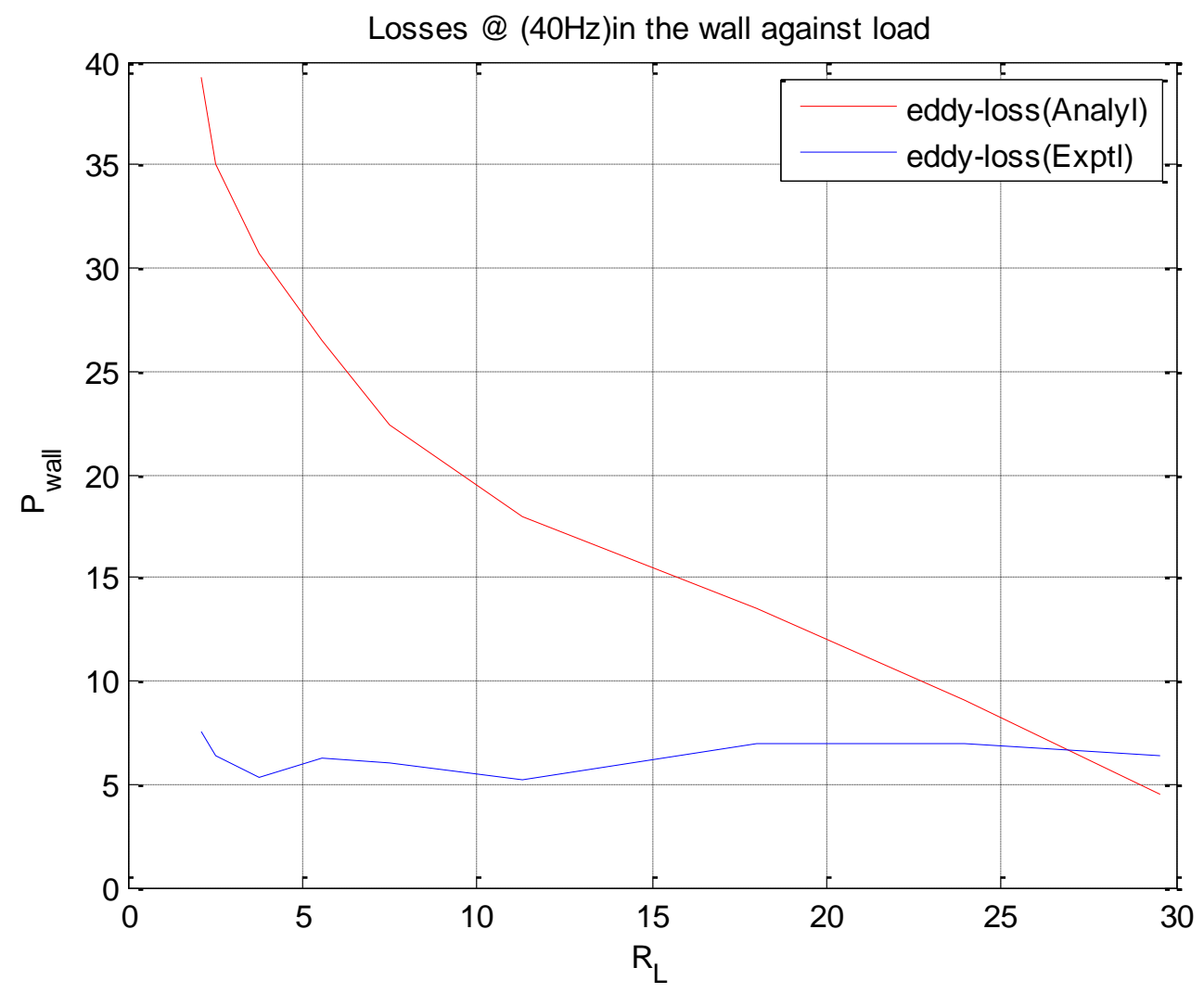

Figure A.3: Wall losses as a function of Load at $40 \mathrm{~Hz}$

\section{A4: Eddy current Losses at $\mathbf{5 0 H z}$ when varying Load resistance}

Clc

clear all

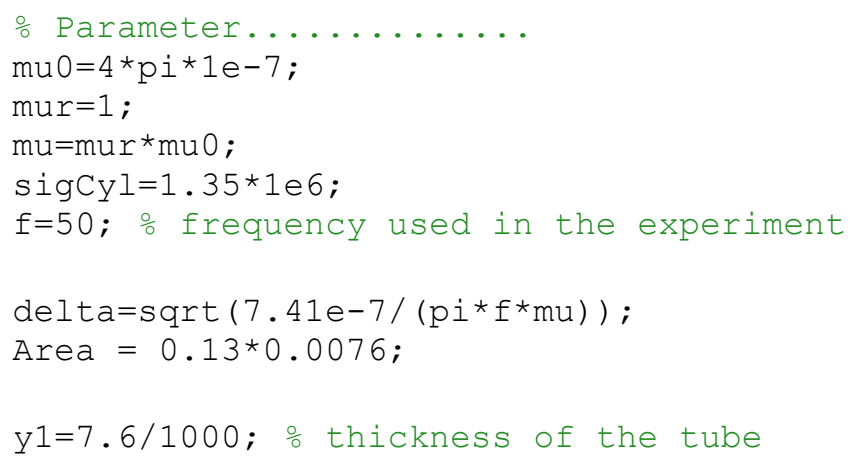




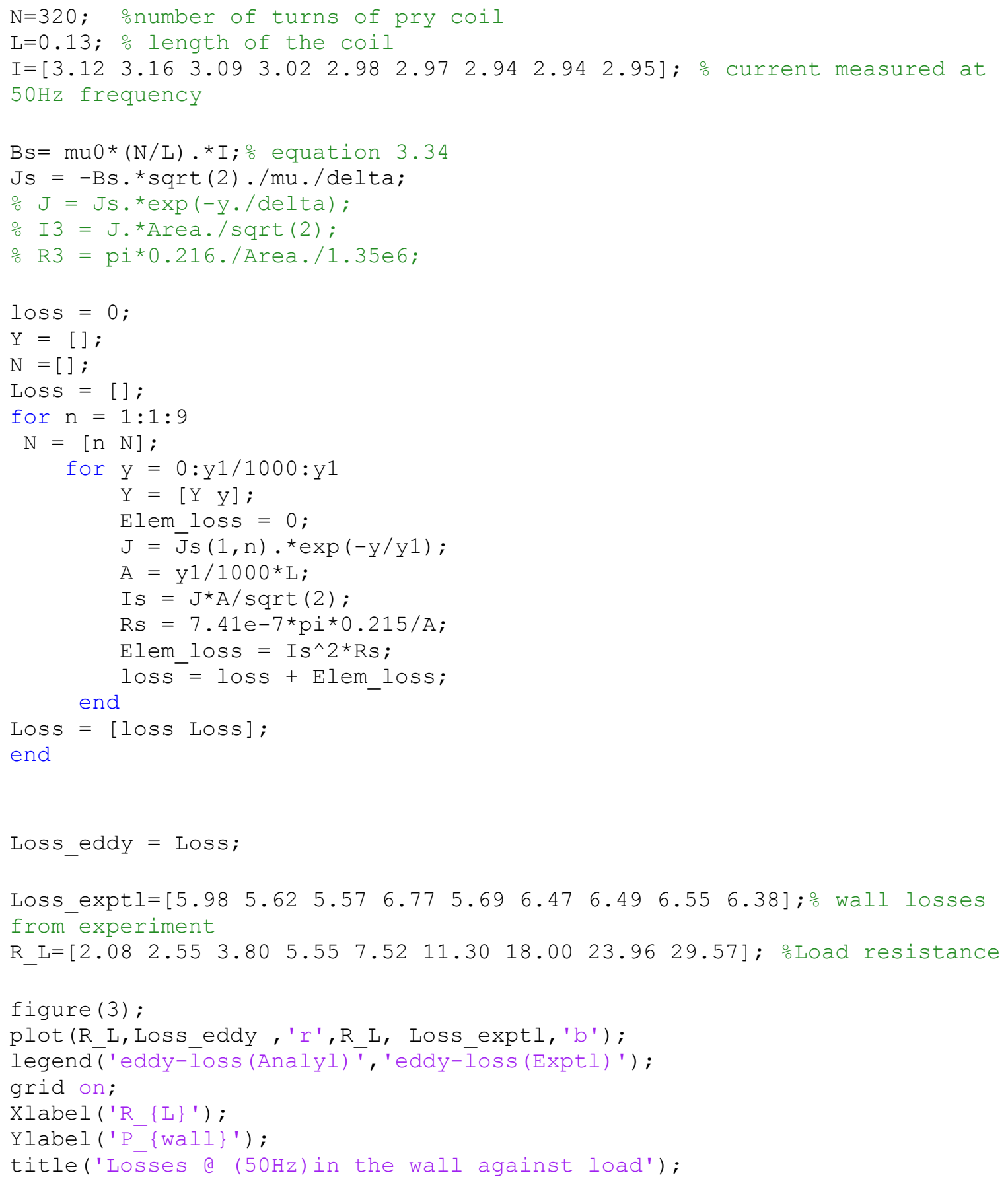




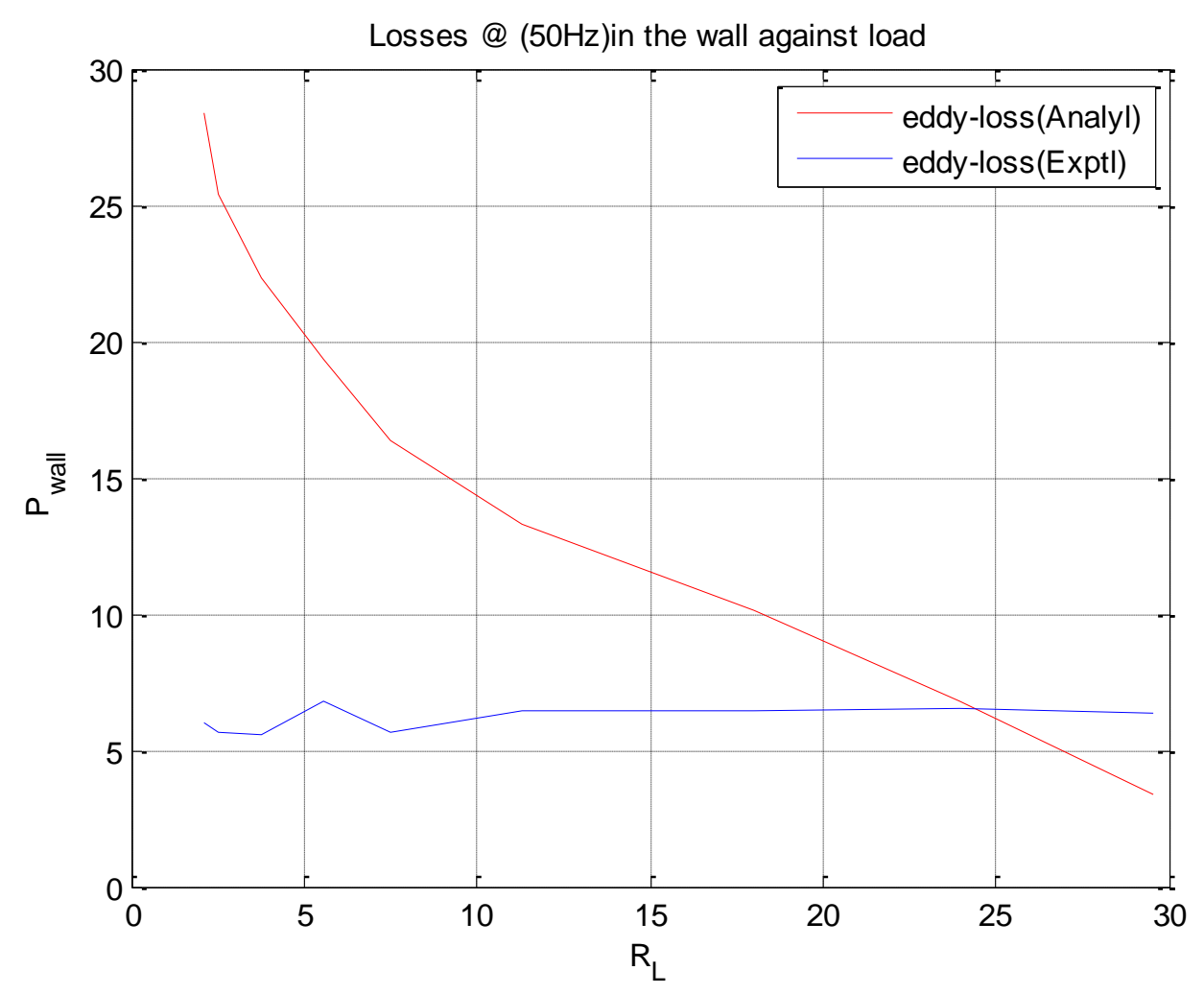

Figure A.4: Wall losses as a function of Load at $50 \mathrm{~Hz}$

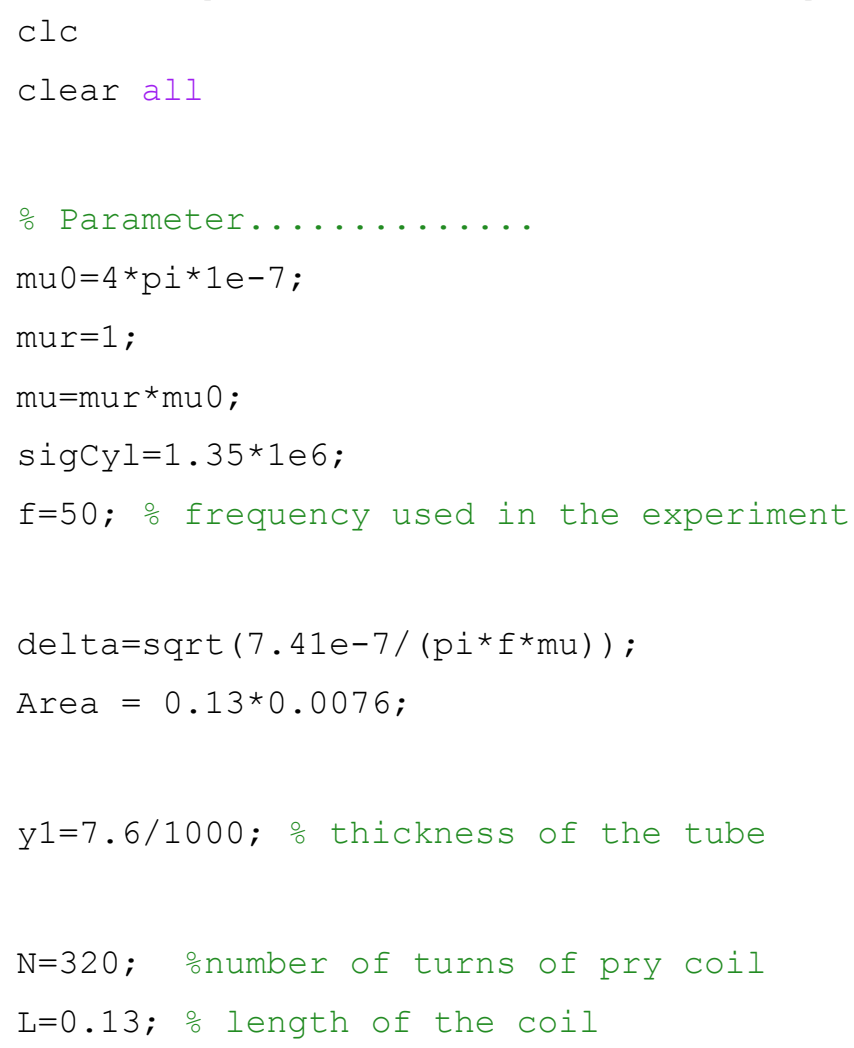




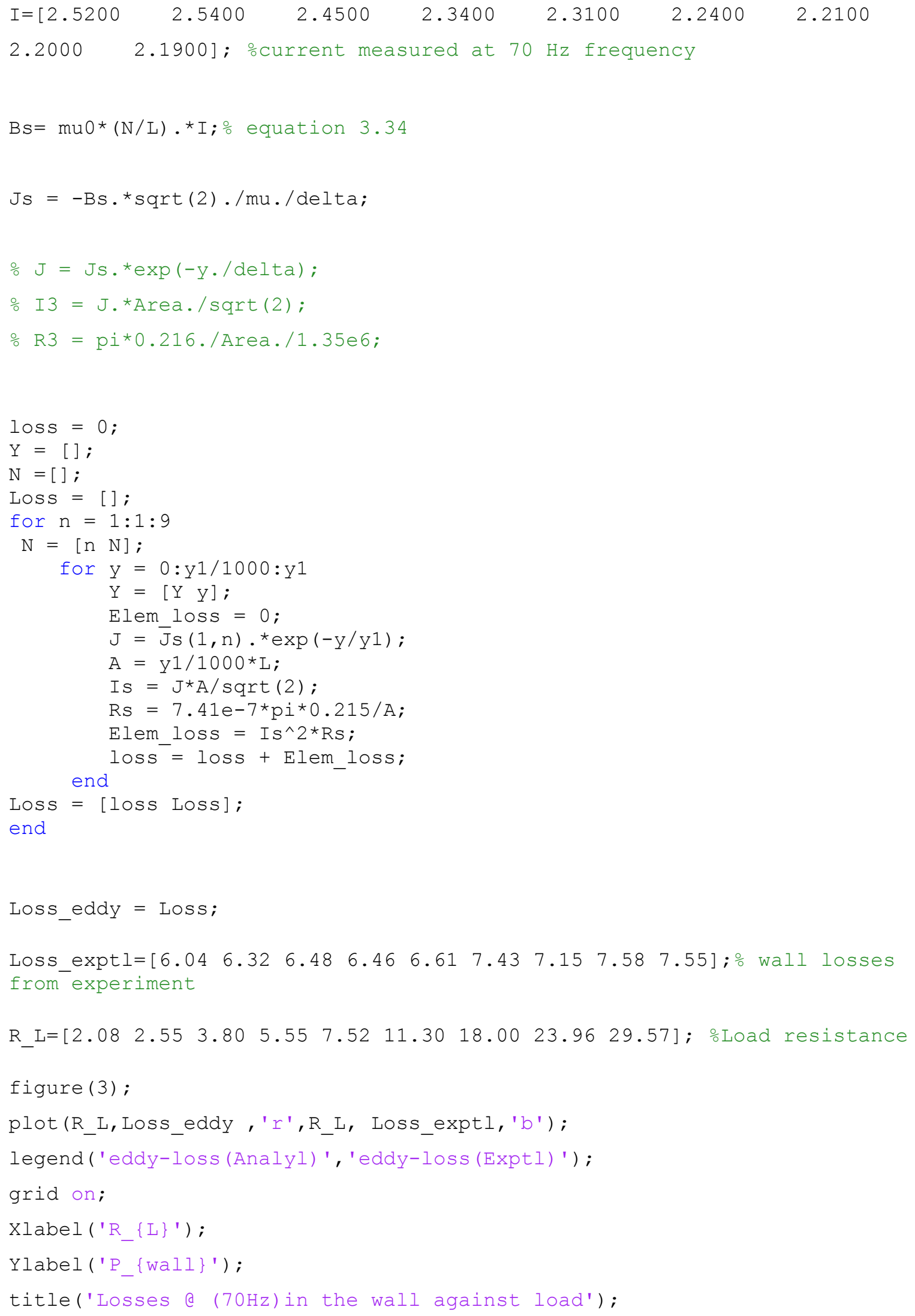




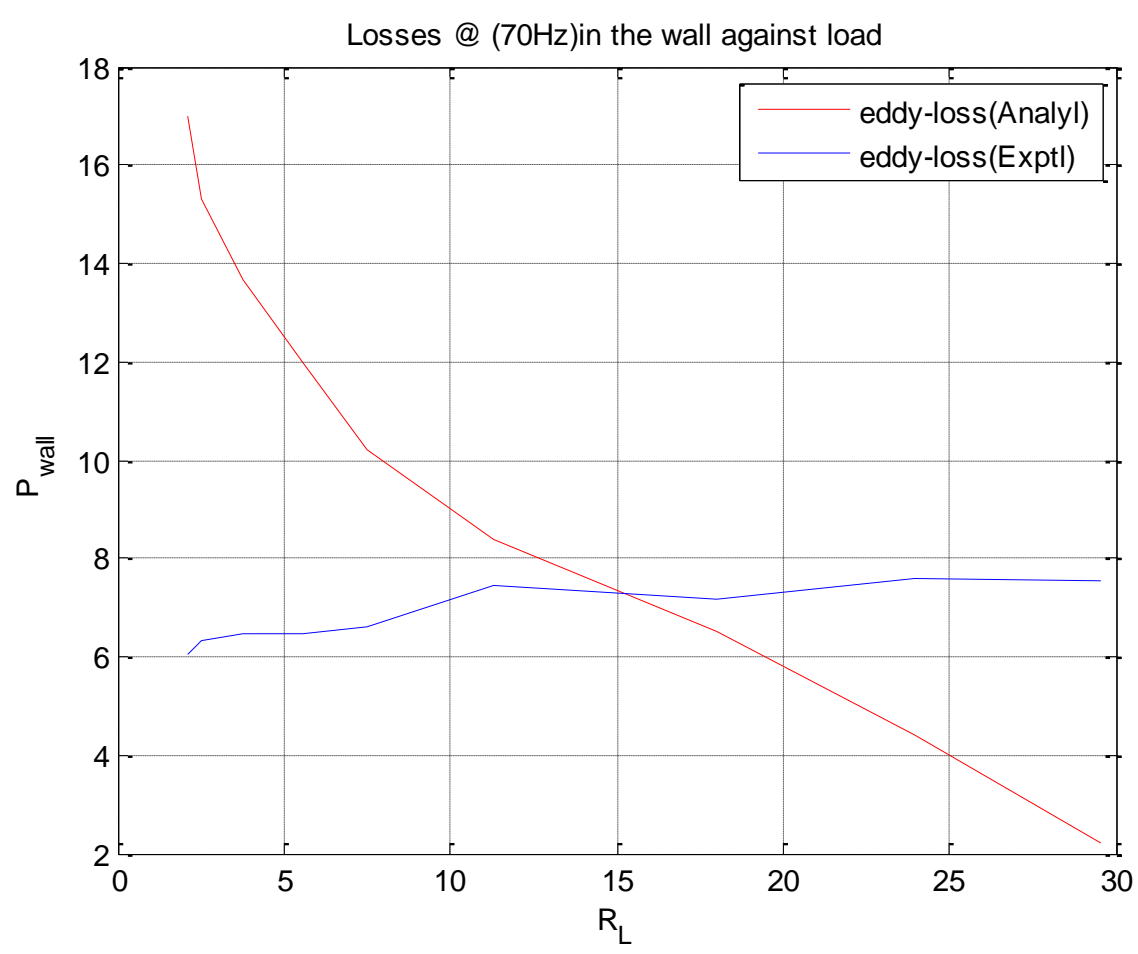

Figure A.5: Wall losses as a function of $\mathrm{Load}$ at $70 \mathrm{~Hz}$

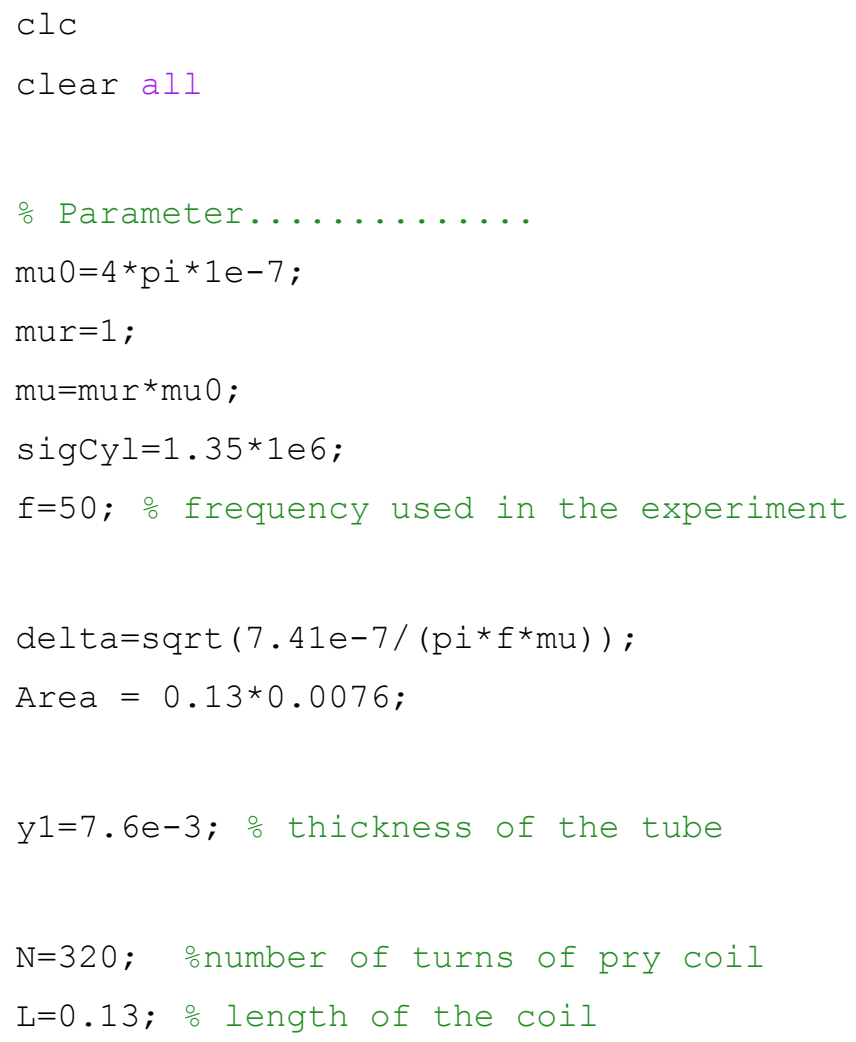




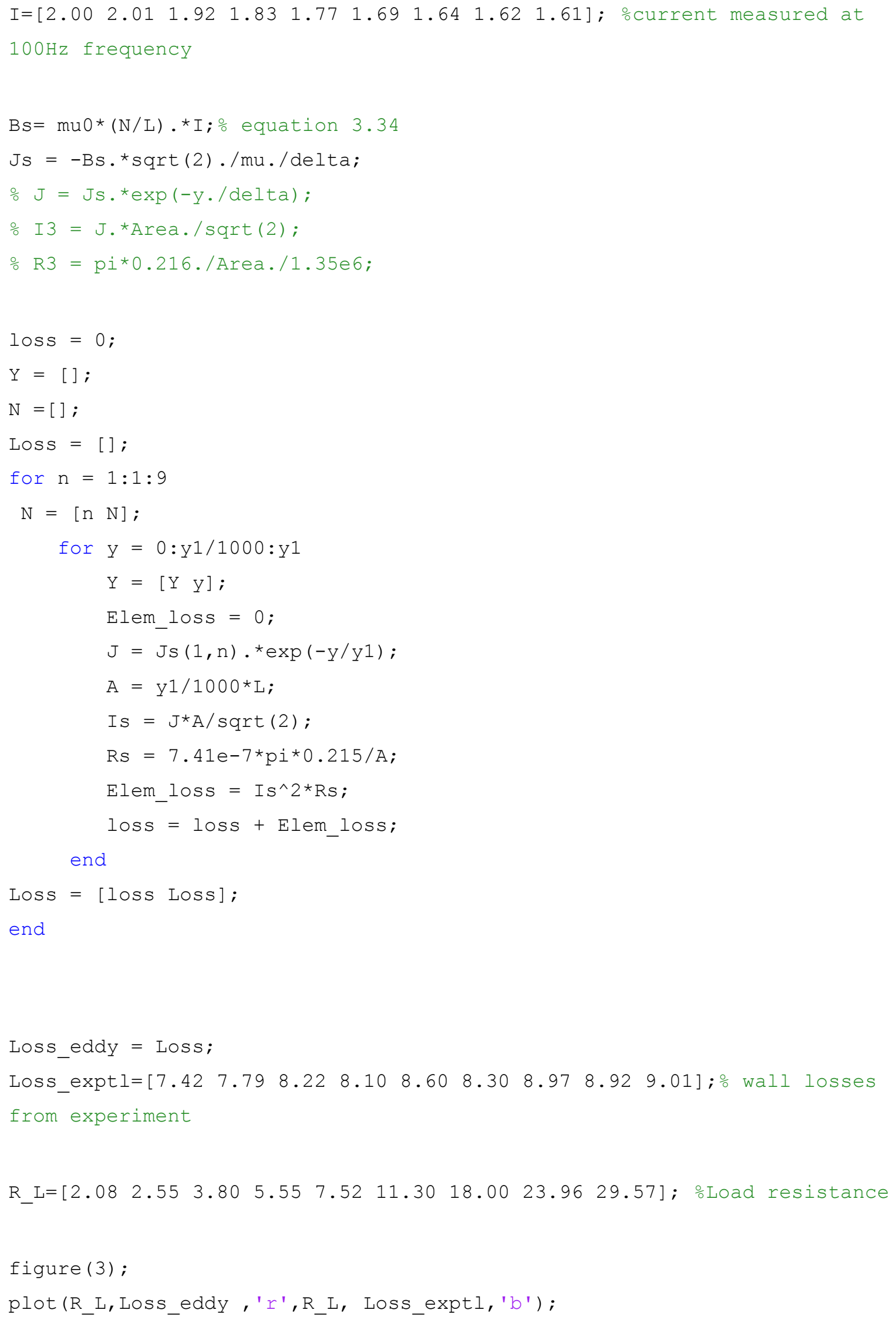


legend ('eddy-loss (Analyl) ', 'eddy-loss (Exptl) ');

grid on;

Xlabel ('R_ $\{\mathrm{L}\}$ ') ;

Ylabel ('P_\{wall\}');

title('Losses a (100Hz)in the wall against load');

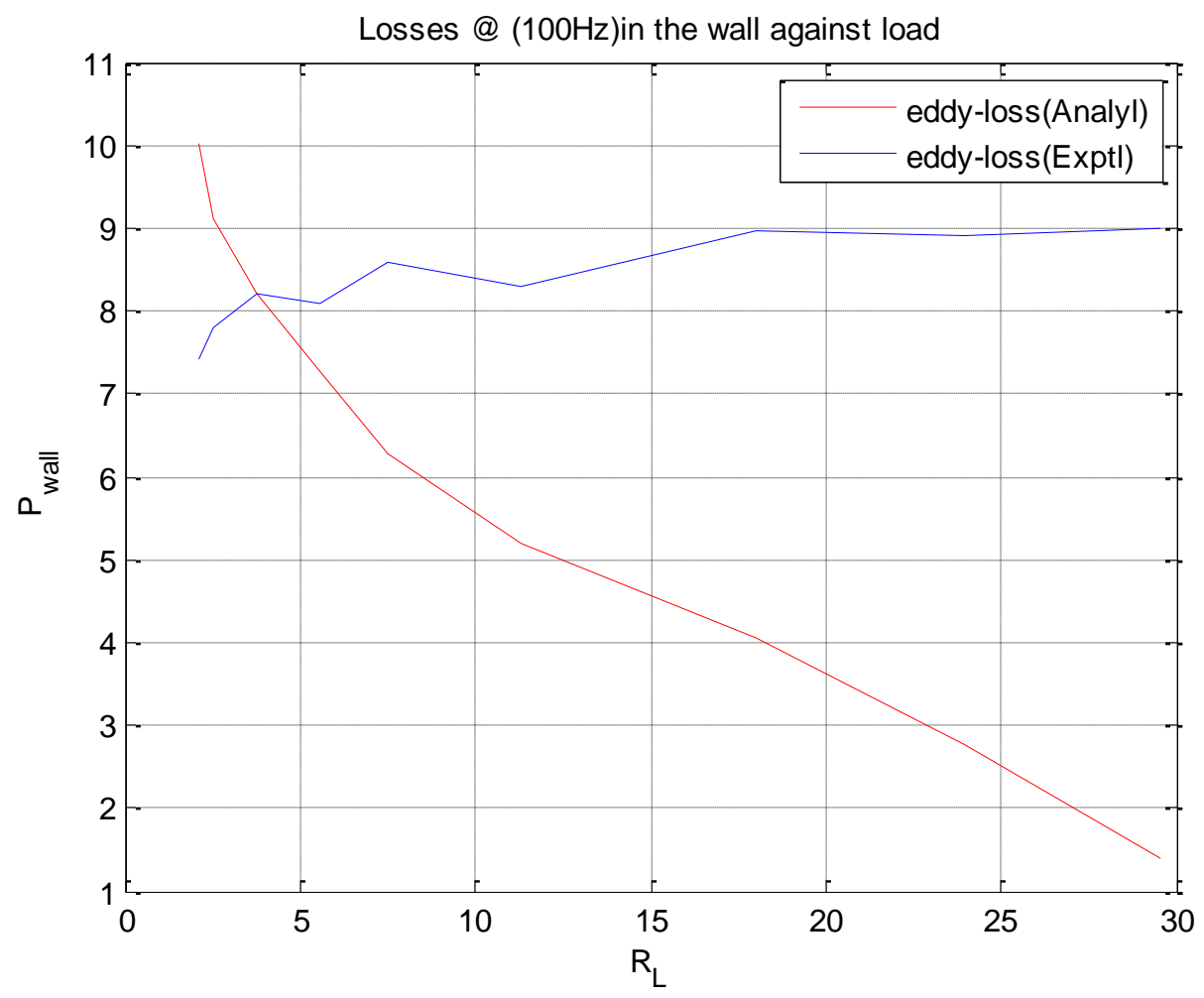

Figure A.6: Wall losses as a function of Load at $100 \mathrm{~Hz}$

\section{A7: No-Load Losses in the wall as a function of frequency}

$\mathrm{clc}$

clear all

\% Parameter..............

$\mathrm{mu} 0=4 * \mathrm{pi} * 10^{\wedge}-7$;

$\operatorname{mur}=1$;

mu $=$ mur $*$ mu 0 ;

$\operatorname{sigCyl}=1 \cdot 35 * 10^{\wedge} 6$;

$\mathrm{f}=\left[\begin{array}{llllllll}40 & 50 & 70 & 100 & 200 & 300 & 500 & 1000\end{array}\right]$; $\%$ frequency used in the experiment delta $=1 . / \operatorname{sqrt}\left(\mathrm{pi} .{ }^{*} \mathrm{f} \mathrm{mu}^{*} \mathrm{sigCyl}\right)$;

odelta $=7.7 e-3 ;$

$\mathrm{y} 1=0.008 ; \%$ Thickness of tube

$\mathrm{N}=320$; onumber of turns of pry coil

$\mathrm{L}=0.13 ; \%$ length of he coil

$I=\left[\begin{array}{llllllll}3.63 & 2.98 & 2.20 & 1.60 & 0.895 & 0.669 & 0.478 & 0.300\end{array}\right] ;$ onolload current

measured at each frequency

LoSS $=[] ;$

$\mathrm{Bs}=\mathrm{mu} 0 \star(\mathrm{N} . / \mathrm{L}) .{ }^{\star} \mathrm{I} ; \%$ equation 3.34 


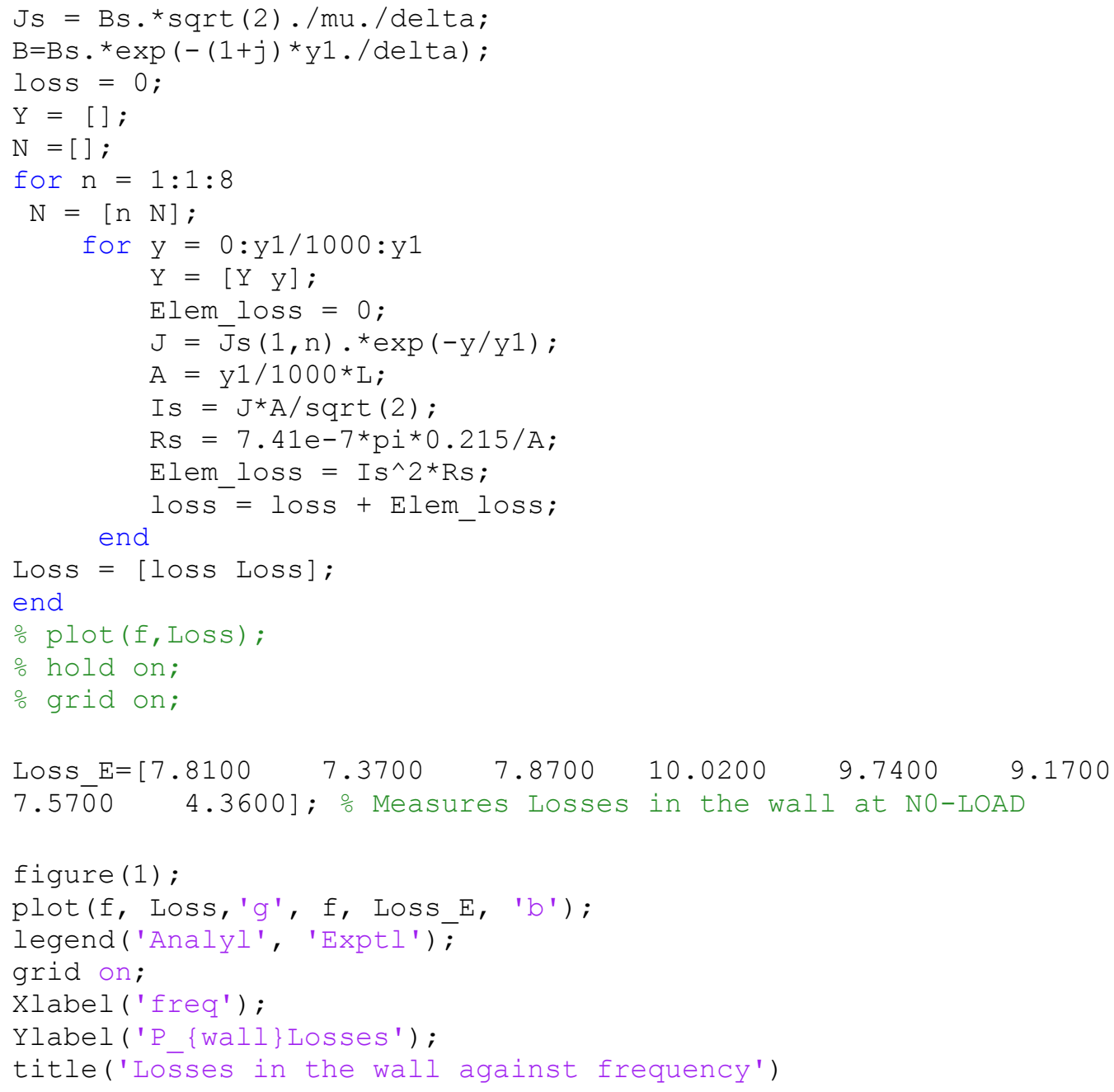




\section{Appendix B}

\section{Pictures form the laboratory}

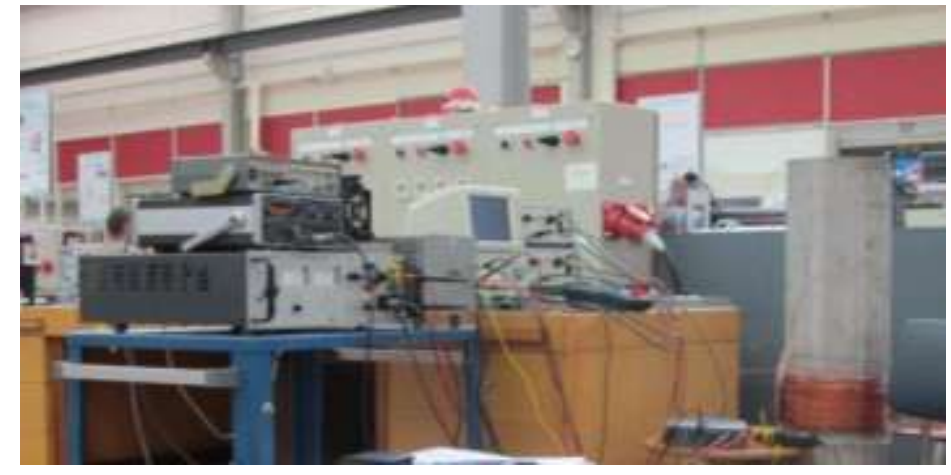

Figure B.1: picture of experiment in the laboratory

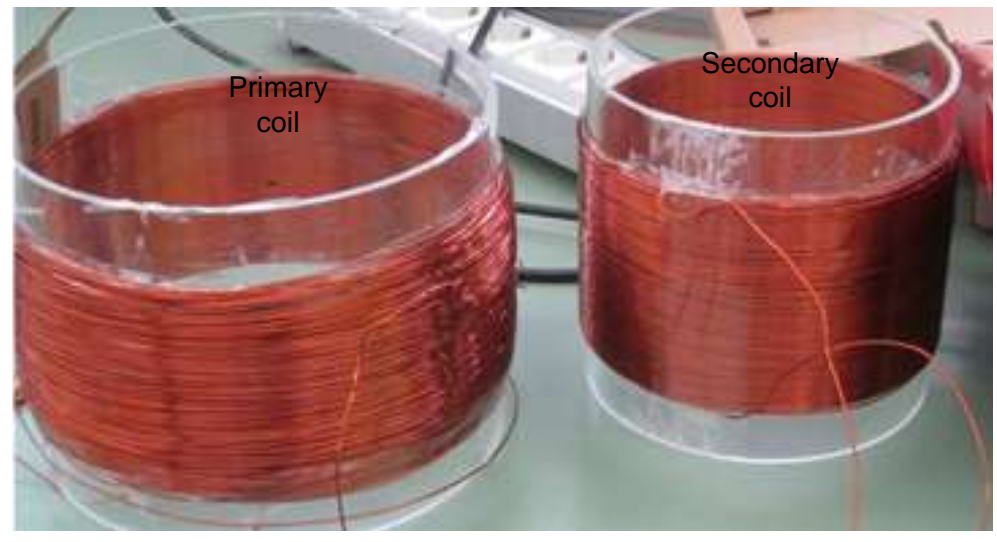

Figure B.2: picture of primary and secondary coil used

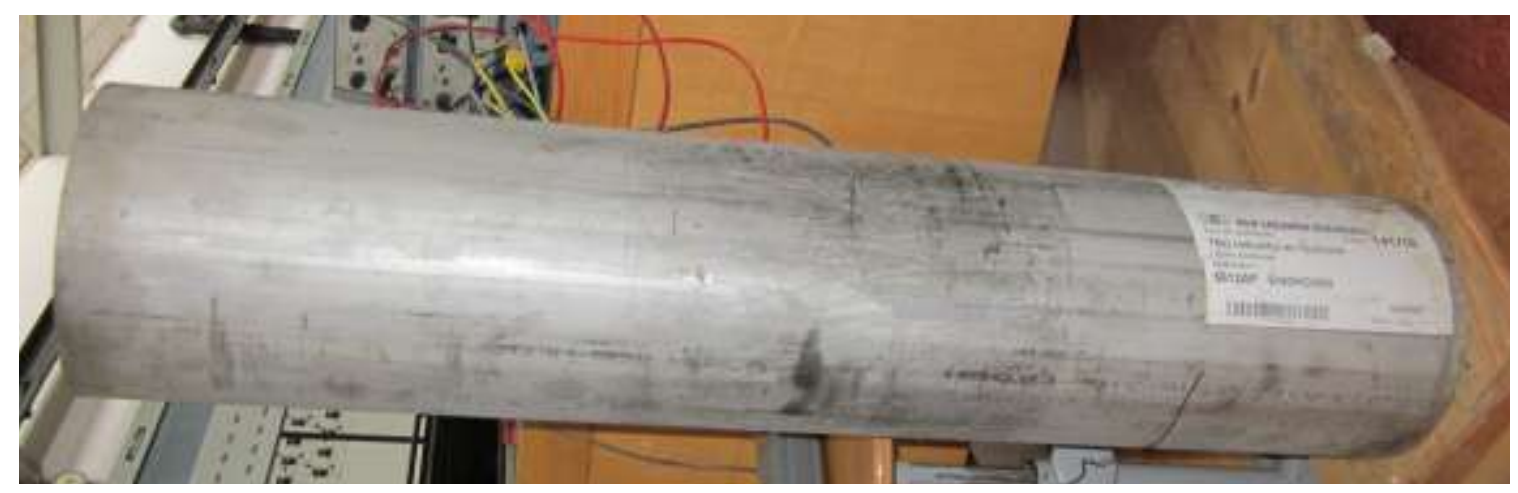

Figure B.3: Stainless steel pipe 


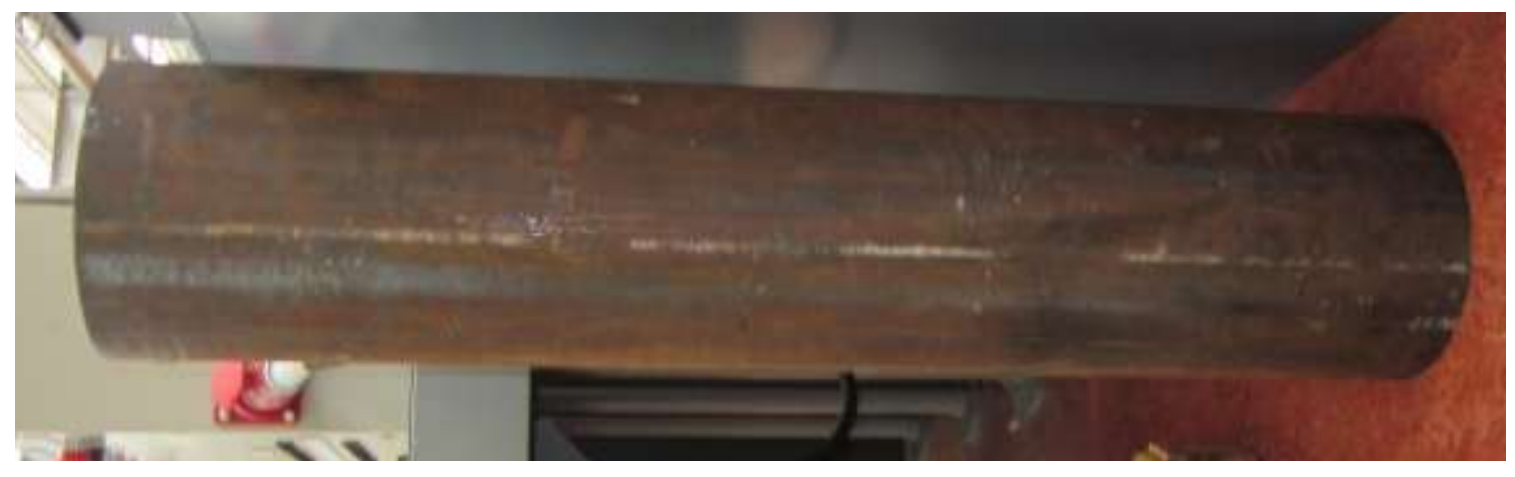

Figure B.4: Ferro-steel pipe 


\section{Appendix C}

\section{Skin depth at different frequency}

In this appendix the skin depth of both stainless-steel and ferro-steel material are given at different frequencies considered in this thesis research.

The expression for the skin depth $\delta=\sqrt{2 /(\omega \mu \sigma)}$ is given in equation 3.19

This can be simplify further as

$$
\delta=\frac{1}{\sqrt{\pi f \mu \sigma}}
$$

Where $\sigma$ and $\mu=\mu_{0} \mu_{r}$ are conductivity and permeability of the material respectively

\section{C1 Skin depth of Stainless-steel (316L)}

Stainless steel material of grade 316L has conductivity of $\sigma=1.35 \mathrm{MS} / \mathrm{m}$ and a relative permeability of $\mu_{r} \sim 1$. (see table 4.1). The skin depth of this materials at various frequencies used in this experiment are given in table C.1

Table C.1: skin depth of Stainless steel at frequency considered

\begin{tabular}{|c|c|}
\hline freq [Hz] Skin Depth( $\mathbf{\delta})[\mathbf{m m}]$ \\
\hline 40 & 68.5 \\
\hline 50 & 61.3 \\
\hline 70 & 51.8 \\
\hline 100 & 43.3 \\
\hline 200 & 30.6 \\
\hline 300 & 25.0 \\
\hline 500 & 19.4 \\
\hline 1000 & 13.7 \\
\hline
\end{tabular}

\section{C1 Skin depth of ferro-steel (ST37)}

Ferro steel material of grade ST37 has conductivity of $\sigma=6.25 \mathrm{MS} / \mathrm{m}$ and a relative permeability of $\mu_{r} \sim 500$. (see table 4.1). The skin depth of this materials at various frequencies used in this experiment are given in table C.2 
Table C.1: skin depth of Ferro-steel at frequency considered

\begin{tabular}{|c|c|}
\hline freq [Hz] Skin Depth( $(\boldsymbol{\delta})[\mathbf{m m}]$ \\
\hline 40 & 1.4 \\
\hline 50 & 1.3 \\
\hline 70 & 1.1 \\
\hline 100 & 0.9 \\
\hline 200 & 0.6 \\
\hline 300 & 0.5 \\
\hline 500 & 0.4 \\
\hline 1000 & 0.3 \\
\hline
\end{tabular}

